\title{
Singularidades de famílias de matrizes simétricas
}

\author{
Luis Renato Gonçalves Dias
}




\title{
Singularidades de famílias de matrizes simétricas
}

\author{
Luis Renato Gonçalves Dias
}

\section{Orientadora: Profa. Dra. Ana Claudia Nabarro}

\author{
Dissertação apresentada ao Instituto de Ciências \\ Matemáticas e de Computação - ICMC-USP, como parte \\ dos requisitos para obtenção do título de Mestre em \\ Matemática.
}

USP - São Carlos

Janeiro/2009 
Fos meus queridos pais, 


\section{Agradecimentos}

Primeiramente, agradeço a Deus por ter me dado esta oportunidade.

À minha família, pela base sólida que sempre me deu força para encarar a vida de frente e pelo seu imenso amor. Aos meus pais, Fernando e Jussara, pelos sacrifícios que fizeram para que eu pudesse prosseguir nos estudos.

À minha querida noiva Josiani, por estar sempre do meu lado, com muito amor e carinho.

À professora Ana Claudia Nabarro, os meus mais sinceros agradecimentos, pela sua orientação, dedicação, ensinamentos, incentivos e por ter sido tão importante no meu crescimento profissional.

Ao professor José Carlos de Souza Junior, por ter acreditado no meu trabalho desde a minha graduação, pelos conselhos e incentivos. Sinto apenas que a distância não me tenha permitido usufruir mais de seus ensinamentos e de seu convívio.

Aos professores do ICMC, com os quais muito aprendi, em especial à professora Maria Aparecida Soares Ruas pelo apoio e incentivo.

A todos os colegas e funcionários do ICMC-USP, em especial à Miriam da Silva Pereira pela ajuda.

Ao CNPq, pelo suporte financeiro.

Enfim, a todos que colaboraram direta ou indiretamente para a concretização deste sonho. 


\section{Resumo}

Estudamos singularidades de famílias de matrizes simétricas. O objetivo é classificar as singularidades simples de tais famílias e estudar a geometria de alguns objetos associados a elas. 


\section{Abstract}

We study the singularities of families of symmetric matrices. The aim of this work is to classify simple singularities of such families and study the geometry of some objects associated to them. 


\section{Sumário}

Introdução

1 Teoria de classificação $\quad 3$

1.1 Conceitos da teoria de classificação . . . . . . . . . . . . . . . . . 4

2 Preliminares para a classificação de matrizes $\quad 9$

2.1 Resultados básicos e dualidade . . . . . . . . . . . . . . . . . . . . 9

2.2 Grupo $\mathcal{G} \ldots \ldots \ldots \ldots \ldots$. . . . . . . . . . . . . . . . . 12

2.3 Espaço tangente . . . . . . . . . . . . . . . . . . . . 15

2.4 Determinação Finita . . . . . . . . . . . . . . . . . . . . . 17

2.5 Germes Simples . . . . . . . . . . . . . . . . . . . . 21

2.6 Classificação dos 1-jatos provenientes dos pencils de quadráticas . . . . . . . . 26

3 Classificação $\quad 31$

3.1 Os casos coposto 0 , coposto $1, \mathrm{r}=1 \ldots \ldots \ldots$. . . . . . . . . . . . 31

3.2 Sobre o 1 -jato de posto $1 \ldots \ldots$. . . . . . . . . . . . . . . 34

3.3 Caso $\mathrm{r}=\mathrm{n}=2 \ldots \ldots \ldots \ldots$. . . . . . . . . . . . . . . . . 36

3.4 Caso $\mathrm{r}=2 \mathrm{e} \mathrm{n}=3 \ldots \ldots \ldots \ldots$

3.5 Caso $\mathrm{r}=4 \mathrm{e} \mathrm{n}=3 \ldots \ldots \ldots \ldots \ldots$

4 Geometria dos germes simples $\quad 49$

4.1 Singularidades do discriminante e número de Milnor . . . . . . . . . . . . . . . 50

4.2 Desdobramento Versal . . . . . . . . . . . . . . . . . . . . . 53

4.3 Geometria adicional $(\mathrm{r}=\mathrm{n}=2) \ldots \ldots \ldots \ldots \ldots$

$\begin{array}{ll}\text { A Caracterização geométrica } & 57\end{array}$

B Símbolo Segre $\quad 63$

$\begin{array}{lll}\text { C } \mathcal{K}_{V} \text {-equivalência } & 67\end{array}$

$\begin{array}{ll}\text { Referências } & 69\end{array}$ 



\section{Introdução}

Uma investigação recente na teoria de singularidades diz respeito ao estudo de famílias de matrizes. Em [5], J. W. Bruce estuda as matrizes simétricas. Em [7], J. W. Bruce e F. Tari estudam as matrizes quadradas e em [16], G. J. Haslinger estuda singularidades de matrizes anti-simétricas. A investigação da geometria associada às singularidades de tais matrizes ainda é um problema em aberto de interesse. O artigo recente de V. V. Goryunov e D. Mond [14], mostra que existem fórmulas surpreendentes relacionando os invariantes clássicos, o número de Milnor e o número de Tjurina, de singularidades de matrizes. Esse artigo também abre uma ampla possibilidade de investigação no tema.

O objetivo deste trabalho é estudar famílias de matrizes simétricas, então nossa principal referência é o artigo do Bruce [5]. Uma família de matrizes simétricas é o germe de uma aplicação suave $\left(\mathbb{K}^{r}, 0\right) \rightarrow \operatorname{Sim}_{n}(\mathbb{K})$. Uma motivação para o desenvolvimento do tema é sua relação com a investigação de Equações Diferenciais Binárias (EDB's), que são equações diferenciais da forma

$$
a(x, y) d y^{2}+2 b(x, y) d x d y+c(x, y) d x^{2}=0
$$

onde $a, b, c$ são funções reais suaves de duas variáveis. EDB's são estudadas extensivamente e têm aplicações em geometria diferencial, equações diferenciais parciais e teoria de controle (ver [8],[19]). Por exemplo, as linhas de curvatura ou as linhas assintóticas de uma superfície em $\mathbb{R}^{3}$ são dadas por equações diferenciais binárias.

Dada uma EDB, a correspondente função discriminante $\delta=b^{2}-a c$, desempenha um papel muito importante: a EDB define pares de direções em pontos $(x, y)$ na região do plano onde $\delta>0$, estas direções coincidem sobre o discriminante $\Delta$ dado por $\delta=0$, e a EDB não tem soluções em pontos onde $\delta<0$. Observe que para $r=n=2$, tomando $A=$ $\left(\begin{array}{ll}a(x, y) & b(x, y) \\ b(x, y) & c(x, y)\end{array}\right)$, temos que $\delta$ é a função discriminante de $A$.

No trabalho, exibimos uma relação de equivalência para classificar famílias de matrizes simétricas. Uma das propriedades desta relação é a de que ela preserva a singularidade da função $\delta$ (muito estudada em EDB's). Através desta relação exibimos formas normais para germes simples $\left(\mathbb{K}^{r}, 0\right) \rightarrow \operatorname{Sim}_{n}(\mathbb{K})$ e sua classificação detalhada é a principal parte do trabalho. 
No Capítulo 1, abordamos noções e resultados básicos da teoria de singularidades, como por exemplo, os conceitos de germes, jatos e o método de classificação.

No Capítulo 2, apresentamos alguns conceitos básicos necessários para o estudo dos germes $\left(\mathbb{K}^{r}, 0\right) \rightarrow \operatorname{Sim}_{n}(\mathbb{K})$, como o grupo $\mathcal{G}$ que age nestes germes e suas propriedades. Também analisamos para quais valores de $r$ e $n$, podemos obter germes simples e listamos os 1-jatos para $n=2,3$, que são provenientes da teoria de pencils.

O Capítulo 3 contém a principal parte do trabalho. Através do método da transversal completa, classificamos os germes simples usando os valores de $r$ e $n$ fornecidos no Capítulo 2 e obtivemos formas normais para estes germes.

No Capítulo 4, definimos alguns objetos associados a um germe $A:\left(\mathbb{K}^{r}, 0\right) \rightarrow \operatorname{Sim}_{n}(\mathbb{K})$. Exibimos os tipos de singularidades destes objetos e obtivemos fórmulas que relacionam estas singularidades. Estudamos a geometria dos germes simples obtidos no Capítulo 3, sendo que o caso $r=n=2$ é estudado com mais detalhes, já que está relacionado com EDB's.

O trabalho também contém três apêndices. No primeiro, exibimos alguns critérios de determinação finita não mencionados no texto e também uma prova do critério geométrico para determinação finita em relação ao grupo $\mathcal{G}$, já que para isto foram necessárias novas definições e novos resultados. O segundo apêndice contém uma apresentação do conceito de símbolo segre, que aparece na classificação de pencils. O terceiro contém uma apresentação sobre a $\mathcal{K}_{V}$-equivalência de Damon, que durante o trabalho foi relacionada com a relação de equivalência que usamos para matrizes, garantindo assim que alguns resultados do nosso contexto podem ser deduzidos do trabalho de Damon. 


\section{Capítulo 1}

\section{Teoria de classificação}

Classificar objetos é uma das principais preocupações em matemática. Uma das ferramentas mais utilizadas para isto é uma relação de equivalência apropriada que permita listar os diferentes tipos de objetos que possam aparecer. A motivação para a busca de uma forma simples para o representante de uma classe de equivalência é a de que tal modelo possui todas as propriedades dos elementos de sua classe e os cálculos com esse modelo são mais simples.

Denotamos por $\operatorname{Sim}_{n}(\mathbb{K})$, o conjunto das matrizes simétricas $n \times n$ com coeficientes no corpo $\mathbb{K}(\mathbb{K}=\mathbb{C}$ ou $\mathbb{R})$. Parte deste trabalho consiste em classificar localmente aplicações suaves $A: \mathbb{K}^{r} \rightarrow \operatorname{Sim}_{n}(\mathbb{K})$, através de uma relação de equivalência apropriada. Para isso, vamos utilizar conceitos e resultados da teoria de singularidades, mais especificamente da teoria de classificação.

$\mathrm{Na}$ teoria de classificação, o objetivo é listar formas normais de germes suaves $f \in$ $\mathcal{M}_{n} \mathcal{O}(n, p)$ sob a ação de um determinado grupo $G$. As ações mais utilizadas para a classificação de germes são dadas pelos grupos de Mather $G=\mathcal{R}, \mathcal{L}, \mathcal{C}, \mathcal{K}$ ou $\mathcal{A}$. Uma das principais idéias na teoria de singularidades é substituir o espaço dos germes $\mathcal{M}_{n} \mathcal{O}(n, p)$ pelo espaço dos jatos $J^{k}(n, p)$ e assim a classificação é feita indutivamente em cada nível dos $k$-jatos. Os principais resultados usados para esse método são a Transversal Completa, Lema de Mather e critérios de determinação finita. Veremos que a Transversal Completa é usada para construir cada $k$-jato do representante de um dado germe. Começando pelo 1-jato e passando em cada nível até chegarmos em seu $k$-jato, sendo $k$ igual ao grau de determinação do germe $f$, podendo ter possivelmente alguns destes níveis vazios. Em cada nível, ela é alternada com o uso do Lema de Mather para que se possa ir obtendo representantes de todas as possíveis órbitas. Vamos utilizar este mesmo método para a classificação de famílias de matrizes simétricas utilizando uma ação apropriada para este caso, porém menos conhecida, que será apresentada no Capítulo 2.

Inicialmente, apresentemos os conceitos gerais para este método (ver [13] ou [24]). 


\subsection{Conceitos da teoria de classificação}

Introduzimos no conjunto de todas as aplicações suaves definidas em uma vizinhança de $x$ em $\mathbb{K}^{n}$ e com valores em $K^{p}$ a seguinte relação de equivalência:

Definição 1.1.1. Duas aplicações suaves $f: U \subset \mathbb{K}^{n} \rightarrow \mathbb{K}^{p}$ e $g: V \subset \mathbb{K}^{n} \rightarrow \mathbb{K}^{p}$ são equivalentes quando existir uma vizinhança $W \subset U \cap V$ de $x$ em $\mathbb{K}^{n}$ tal que as restrições $f_{\mid W}$ e $g_{\mid W}$ coincidem.

As classes de equivalência desta relação são chamadas de germes de aplicações em $x$ e um elemento dessa classe é chamado de representante do germe. Se $f$ e $g$ são representantes do mesmo germe então devemos ter $f(x)=g(x)$ e assim qualquer outro representante deve assumir o mesmo valor em $x$, digamos $y$. Adotamos a notação $f:\left(\mathbb{K}^{n}, x\right) \rightarrow\left(\mathbb{K}^{p}, y\right)$, para indicar o germe de aplicação em $x$. Sem perda de generalidade tomamos $x=0$.

$\mathrm{O}$ conjunto dos germes $\left(\mathbb{K}^{n}, 0\right) \rightarrow \mathbb{K}^{p}$ é denotado por $\mathcal{O}(n, p)$. Quando $p=1$ (germes de funções $), \mathcal{O}(n, 1)$ é denotado apenas por $\mathcal{O}_{n}$. Usando as operações de soma e multiplicação de $\mathbb{K}$ temos que $\mathcal{O}_{n}$ possui uma estrutura de anel comutativo com identidade. Este anel é um anel local (possui um único ideal maximal), cujo maximal é dado por $\mathcal{M}_{n}=\{f \in$ $\left.\mathcal{O}_{n} / f(0)=0\right\}$. Quando não houver perigo de confusão, denotamos $\mathcal{O}_{n}, \mathcal{M}_{n}$ apenas por $\mathcal{O}$, $\mathcal{M}$, respectivamente.

Definição 1.1.2. O espaço dos $k$-jatos, $J^{k}(n, p)$, é o $\mathbb{K}$-espaço vetorial das aplicações $f$ : $\mathbb{K}^{n} \rightarrow \mathbb{K}^{p}$, onde cada componente de $f$ é um polinômio de grau $\leqslant k$ nas variáveis $x_{1}, \ldots, x_{n}$ com termo constante nulo.

Temos que $J^{k}(n, p)$ é um espaço vetorial de dimensão finita e que

$$
J^{k}(n, p)=\mathcal{M}_{n} \mathcal{O}(n, p) / \mathcal{M}_{n}^{k+1} \mathcal{O}(n, p) .
$$

A cada $f \in \mathcal{M}_{n} \mathcal{O}(n, p)$ associamos o $k$-jato $j^{k} f \in J^{k}(n, p)$, que é o polinômio de Taylor de $f$ de grau $\leqslant k$.

A teoria de classificação tem como objetivo agrupar os elementos de $\mathcal{M}_{n} \mathcal{O}(n, p)$ através de uma relação de equivalência. Para isto, necessitamos da noção de ação de um grupo e de grupo de Lie.

Definição 1.1.3 (Ação de Grupos). Uma ação de um grupo G em um conjunto $M$ é uma aplicação $\Phi: G \times M \rightarrow M$, satisfazendo:

a) $\Phi(1, x)=x, \forall x \in M$,

b) $\Phi(g h, x)=\Phi(g, \Phi(h, x)), \forall x \in M e \forall g, h \in G$, 
onde 1 é a identidade de $G$.

Observação 1.1.4. Geralmente escrevemos $\Phi(g, x)=g . x$.

Dada uma ação $\Phi$, podemos definir uma relação de equivalência $\sim$ em $M$. Dizemos que $x \sim y$ se existe $g \in G$ tal que $y=g . x$. Dado $x \in M$, a órbita de $x$ é por definição a classe de equivalência que contém $x$, ou seja, o conjunto

$$
G . x=\{g . x ; g \in G\} .
$$

Assim, dizemos que dois elementos são $G$-equivalentes se eles pertencem à mesma órbita.

Definição 1.1.5 (Grupo de Lie). Um grupo de Lie é uma variedade diferenciável G, de classe $C^{\infty}$, dotada de uma estrutura de grupo em que a multiplicação $m: G \times G \rightarrow G$, $m\left(g_{1}, g_{2}\right)=g_{1} g_{2}$ e a inversão $i: G \rightarrow G, i(g)=g^{-1}$, são aplicações de classe $C^{\infty}$.

Uma ação suave de um grupo de Lie em uma variedade suave $M$ é uma ação

$$
\Phi: G \times M \rightarrow M
$$

em que $\Phi$ é uma aplicação suave.

Proposição 1.1.6. Seja $\Phi: G \times M \rightarrow M$ uma ação suave de um grupo de Lie em uma variedade diferenciável $M$. Suponha que as órbitas são subvariedades suaves de $M$. Então para qualquer $x \in M$ a aplicação $\Phi_{x}: G \rightarrow G . x$, dada por $\Phi_{x}(g)=g . x$, é uma submersão.

Demonstração. Ver ([13], p. 74).

Corolário 1.1.7. Nas condições da Proposição 1.1.6, temos que o espaço tangente à órbita G.x em $x$ é a imagem de $d_{1} \Phi_{x}: T_{1} G \rightarrow T_{x}(G . x)$, ou seja, $T_{x} G . x=d_{1} \Phi_{x}\left(T_{1} G\right)$.

\section{Relações de equivalência dos germes.}

As relações de equivalência mais utilizadas para o problema de classificação de germes são as $G$-equivalências, onde $G$ é um dos grupos de Mather $\mathcal{R}, \mathcal{L}, \mathcal{C}, \mathcal{K}$ ou $\mathcal{A}$. Dentre estes, os que nos interessam são os grupos $\mathcal{R}, \mathcal{C}$ e $\mathcal{K}$.

Definição 1.1.8. Denotamos por $\mathcal{R}$ o grupo dos germes de difeomorfismos $h:\left(\mathbb{K}^{n}, 0\right) \rightarrow$ $\left(\mathbb{K}^{n}, 0\right)$.

Este grupo é chamado grupo de mudanças de coordenadas na fonte e age sobre $\mathcal{M}_{n} \mathcal{O}(n, p)$ por composição à direita

$$
\begin{aligned}
\mathcal{R} \times \mathcal{M}_{n} \mathcal{O}(n, p) & \longrightarrow \mathcal{M}_{n} \mathcal{O}(n, p) \\
(h, f) & \longmapsto f \circ h^{-1} .
\end{aligned}
$$


Definição 1.1.9. Sejam $f, g \in \mathcal{M}_{n} \mathcal{O}(n, p)$. Dizemos que $f$ é $\mathcal{R}$-equivalente a $g$ se existe $h \in \mathcal{R}$ tal que $f \circ h^{-1}=g$.

Definição 1.1.10. O grupo de contato, que denotamos por $\mathcal{K}$, é o grupo dos germes de difeomorfismos $\left(\mathbb{K}^{n} \times \mathbb{K}^{p}, 0\right) \rightarrow\left(\mathbb{K}^{n} \times \mathbb{K}^{p}, 0\right)$, que são da forma

$$
H(x, y)=(h(x) ; \Theta(x, y))
$$

onde $h \in \mathcal{R}$ e $\Theta(x, 0)=0$, para $x$ próximo da origem.

Definição 1.1.11. Dois germes $f, g \in \mathcal{M O}(n, p)$ são $\mathcal{K}$-equivalentes quando existir $H=$ $(h, \Theta) \in \mathcal{K}$, tal que:

$$
(h(x), g \circ h(x))=H(x, f(x)),
$$

isto é, de uma forma especial, $H$ leva o gráfico de $f$ sobre o gráfico de $g$.

Denotamos por $\mathcal{C}$ o subgrupo de $\mathcal{K}$ formado pelos elementos da forma $H=\left(i d_{n}, \Theta\right)$, onde $i d_{n}$ é a aplicação identidade de $\mathbb{K}^{n}$. Da mesma forma, temos que dois germes $f, g \in \mathcal{M O}(n, p)$ são $\mathcal{C}$-equivalentes quando existir $H \in \mathcal{C}$ tal que

$$
H(x, f(x))=(x, \Theta(x, f(x)))=(x, g(x))
$$

Observação 1.1.12. Podemos ver $\mathcal{K}$ como um produto semi-direto dos grupos $\mathcal{R}$ e $\mathcal{C}$. Ver ([13], p. 150).

Lema 1.1.13. Dois germes $f, g \in \mathcal{M O}(n, p)$ são $\mathcal{K}$-equivalentes se, e somente se, existe um germe invertivel $h:\left(\mathbb{K}^{n}, 0\right) \rightarrow\left(\mathbb{K}^{n}, 0\right)$ para o qual os germes $f \circ h$ e g são $\mathcal{C}$-equivalentes.

Proposição 1.1.14. As seguintes duas condições sobre dois germes $f, g \in \mathcal{M O}(n, p)$ são equivalentes:

(i) $f, g$ são $\mathcal{C}$-equivalentes,

(ii) Existe uma matriz $p \times p$ invertivel $\mathcal{U}=\left(u_{i j}\right)$ em que $u_{i j} \in \mathcal{O}_{n}$, tal que

$$
f_{i}(x)=\sum_{j=1}^{p} u_{i j}(x) g_{j}(x), \text { para } 1 \leqslant i \leqslant p .
$$

Demonstração. Ver ([13], p. 145).

O espaço tangente à órbita de um dado germe $f$ pela ação de $G$, onde $G=\mathcal{R}, \mathcal{K}, \mathcal{C}$, é dado por:

$$
T \mathcal{R} . f=\mathcal{M}_{n}\left\{\frac{\partial f}{\partial x_{1}}, \ldots, \frac{\partial f}{\partial x_{n}}\right\}, T \mathcal{C} f=f^{*}\left(\mathcal{M}_{p}\right) \mathcal{O}(n, p) \text { e } T \mathcal{K} f=T \mathcal{R} . f+T \mathcal{C} f .
$$

Definição 1.1.15. Um germe $f \in \mathcal{M}_{n} \mathcal{O}(n, p)$ é $k$-G-determinado se para todo germe $g \in$ $\mathcal{M}_{n} \mathcal{O}(n, p)$ tal que $j^{k} g=j^{k} f$ tem-se que $g$ é $G$-equivalente a $f$. Dizemos que um germe $f$ é finitamente determinado se é $k$-determinado para algum $k$. 
Lema 1.1.16. Sejam $f, g \in \mathcal{M}_{n} \mathcal{O}(n, p)$. Se $j^{k} f$ e $j^{k} g$ não são $J^{k} G$-equivalentes em $J^{k}(n, p)$ então $j^{m} f$ e $j^{m} g$ não são $J^{m} G$-equivalentes em $J^{m}(n, p), \forall m \geqslant k$.

A determinação finita significa que um germe finitamente determinado é equivalente a um de seus polinômios de Taylor e assim o problema da classificação pode ser reduzido ao espaço dos $k$-jatos, que é um espaço de dimensão finita.

Observemos que $\mathcal{O}(n, p)$ não é uma variedade diferenciável e muito menos $G=\mathcal{R}, \mathcal{K}, \mathcal{C}$ são grupos de Lie. Para cada $f \in \mathcal{M}_{n} \mathcal{O}(n, p)$ associamos o $k$-jato $j^{k} f$. A ação de $G$ sobre $\mathcal{M}_{n} \mathcal{O}(n, p)$ fornece uma ação de $J^{k} G$ sobre $J^{k}(n, p)$ que é uma ação de grupo de Lie em um espaço vetorial. A idéia é estudar a ação de $G$ sobre $\mathcal{M}_{n} \mathcal{O}(n, p)$ através da ação de $J^{k} G$ sobre $J^{k}(n, p)$. A classificação é feita indutivamente em cada nível, ou seja, em cada $k$-jato de $f$. As principais ferramentas para este método são a Transversal Completa, o Lema de Mather e os critérios de determinação finita.

Teorema 1.1.17 (Transversal Completa). Seja $f$ um $k$-jato em $J^{k}(n, p)$, escrevemos $G_{1}$ para o subgrupo de $G$ dos elementos cujo 1-jato é a identidade, e $H^{k+1}(n, p)$ para as aplicações polinomiais homogêneas de grau $k+1$. Suponha que $T_{f} J^{k+1} G_{1} . f \cap H^{k+1}(n, p)+T=H^{k+1}(n, p)$ para algum subespaço $T$ de $H^{k+1}(n, p)$. Então todo $(k+1)$-jato com k-jato $f$ é $J^{k+1} G_{1^{-}}$ equivalente a um jato da forma $f+B$, com $B \in T$. Tal $T$ é chamado de transversal completa.

Lema 1.1.18 (Lema de Mather). Seja G um grupo de Lie agindo em uma variedade suave M. Seja $N$ uma subvariedade conexa de $M$ e suponhamos que as órbitas são subvariedades de M. Então $N$ está contida em uma única órbita se, e somente se,

1. $T_{x} N \subset T_{x} G . x, \forall x \in N$.

2. $\operatorname{dim}_{x} G \cdot x=$ constante, $\forall x \in N$.

Demonstração. Ver ([24], p. 8).

Observemos que o Teorema da Transversal Completa é válido para um subgrupo $G_{1}$ de $G$. Como estamos interessados na classificação com o grupo $G$, usamos o Lema de Mather para eliminar os termos desnecessários que não foram eliminados na Transversal Completa com o grupo $G_{1}$.

Mais especificamente, em cada $j^{k} f$ usamos a Transversal Completa para obter uma parametrização dos $(k+1)$-jatos que têm o $k$-jato igual a $j^{k} f$. Podemos usar o Lema de Mather para produzir as $G$-órbitas dentro desta parametrização. Aplicamos o teste de determinação finita a cada órbita no $(k+1)$ )-jato (cujo $k$-jato é $\left.j^{k} f\right)$. Se o germe é $(k+1)$-finitamente determinado paramos o processo. Se não, consideramos o $(k+2)$-jato e seguimos o raciocínio.

Para o estudo dos germes de aplicações $A: \mathbb{K}^{r} \rightarrow \operatorname{Sim}_{n}(\mathbb{K})$, podemos aplicar o método da transversal completa, apresentado acima, usando um determinado grupo. Apresentamos 
no próximo capítulo o grupo que agirá em $A$ e suas propriedades como o espaço tangente à órbita de um dado elemento e critérios para a determinação finita.

Enunciamos a seguir dois resultados de álgebra, que faremos referência durante este trabalho, e que são bastante utilizados na teoria de singularidades.

Lema 1.1.19 (Lema de Nakayama). Sejam $R$ um anel comutativo com identidade 1 e $\mathfrak{M}$ um ideal em $R$ com a propriedade que $1+x$ é invertivel em $R$ para todo $x \in \mathfrak{M}$. Seja $C$ um $R$-módulo e $A, B R$-submódulos de $C$ com A finitamente gerado.

$$
\text { Se } A \subset B+\mathfrak{M} A \text { então } A \subset B \text {. }
$$

Proposição 1.1.20. Seja $C$ um $R$-módulo com base finita e seja $T \subset C$ um $R$-submódulo. Uma condição necessária e suficiente para que $T$ tenha finita codimensão em $C$ é que exista um inteiro $k \geqslant 1$ com $\mathfrak{M}^{k} . C \subset T$.

Demonstração. Ver ([13], p. 104). 


\section{Capítulo 2}

\section{Preliminares para a classificação de matrizes}

Uma família de matrizes simétricas é o germe de uma aplicação suave $\left(\mathbb{K}^{r}, 0\right) \rightarrow \operatorname{Sim}_{n}(\mathbb{K})$. Em [5], J. W. Bruce estudou estas famílias e forneceu uma lista dos germes simples e informações sobre sua geometria. Esta lista foi obtida usando uma relação de equivalência definida por mudanças de coordenadas suaves na fonte e por conjugação parametrizada na meta. Este artigo foi o primeiro de uma série de artigos sobre famílias de matrizes. Em [7], foi dado um tratamento semelhante para famílias de matrizes quadradas em geral e em [16], matrizes anti-simétricas foram estudadas. Famílias de matrizes quadradas também foram estudadas por Arnold em [1], onde as matrizes parametrizadas em $\mathrm{GL}_{n}(\mathbb{K})$ agem por conjugação. Vale observar, que os métodos e ferramentas utilizados para a classificação de famílias de matrizes de [5] e [7] são praticamente análogos.

Nosso trabalho se concentra no estudo de famílias de matrizes simétricas. Neste capítulo, definimos o grupo que age nestes elementos e que nos fornece a relação de equivalência mencionada acima. Será também apresentado o espaço tangente à órbita pela ação desse grupo e critérios de determinação finita. Esses conceitos serão principalmente utilizados no Capítulo 3 , onde classificaremos os germes simples $\left(\mathbb{K}^{r}, 0\right) \rightarrow \operatorname{Sim}_{n}(\mathbb{K})$.

\subsection{Resultados básicos e dualidade}

Apresentamos algumas definições e ferramentas que vão ser utilizadas no estudo das aplicações $A:\left(\mathbb{K}^{r}, 0\right) \rightarrow \operatorname{Sim}_{n}(\mathbb{K})$. Vamos iniciar com uma breve discussão sobre formas bilineares simétricas/quadráticas. Logo após, apresentamos alguns resultados de dualidade. Estes conceitos serão úteis principalmente na classificação das aplicações $A: \mathbb{K}^{r} \rightarrow \operatorname{Sim}_{n}(\mathbb{K})$ ao nível do 1-jato. As referências para essa seção são [15] e [21]. 
Começamos definindo a seguinte ação de $G L_{n}(\mathbb{K})$ em $\operatorname{Sim}_{n}(\mathbb{K})$ :

$$
\begin{aligned}
\Psi: \quad G L_{n}(\mathbb{K}) \times \operatorname{Sim}_{n}(\mathbb{K}) & \longrightarrow \operatorname{Sim}_{n}(\mathbb{K}) \\
(X, A) & \longmapsto\left(X^{-1}\right)^{t} A X^{-1}
\end{aligned}
$$

Dizemos que duas matrizes $A, B \in \operatorname{Sim}_{n}(\mathbb{K})$ são $G L_{n}(\mathbb{K})$-equivalentes quando pertencem à mesma órbita pela ação $\Psi$.

Proposição 2.1.1. Quando $\mathbb{K}=\mathbb{C}$, a ação acima possui $n+1$ órbitas e podemos escolher como representante para cada órbita o elemento $I_{t}^{*}$, onde $I_{t}^{*}$ é a matriz diagonal com 1 nas primeiras t-posições da diagonal e zero nas demais posições.

Proposição 2.1.2. O espaço tangente à órbita pela ação $\Psi$ em um ponto $A \in \operatorname{Sim}_{n}(\mathbb{K})$, isto é $T_{A}\left(G L_{n} . A\right)$, é o $\mathbb{K}$-espaço vetorial dado por $\left\{B^{t} A+A B ; B \in M_{n}(\mathbb{K})\right\}$, onde $M_{n}(\mathbb{K})$ é conjunto das matrizes $n \times n$ com coeficientes $\mathrm{em} \mathbb{K}$.

Demonstração. Pelo Corolário 1.1.7 temos $T_{A}\left(G L_{n} \cdot A\right)=d_{I} \Psi_{A}\left(T_{I} G L_{n}\right)$, onde $I$ denota matriz identidade $n \times n$ e

$$
\begin{aligned}
\Psi_{A}: G L_{n} & \rightarrow G L_{n} . A \\
X & \mapsto X^{t} A X .
\end{aligned}
$$

Lembrando que $T_{I} G L_{n}=M_{n}$, temos:

$$
\begin{aligned}
d_{I} \Psi_{A}: M_{n} & \rightarrow T_{A} G L_{n} \cdot A \\
B & \mapsto B^{t} A+A B,
\end{aligned}
$$

e o resultado segue.

Observação 2.1.3. Seja $A \in \operatorname{Sim}_{n}(\mathbb{K})$ uma matriz invertível. Dado $B \in \operatorname{Sim}_{n}(\mathbb{K})$, temos $\left(\frac{A^{-1} B}{2}\right)^{t} A+A\left(\frac{A^{-1} B}{2}\right)=B \Rightarrow B \in T_{A}\left(G L_{n} . A\right)$. Como $B$ é arbitrário, segue que $T_{A}\left(G L_{n} . A\right)=\operatorname{Sim}_{n}(\mathbb{K})$ e assim a órbita de $A$ é um conjunto aberto de $\operatorname{Sim}_{n}(\mathbb{K})$.

Denotamos por $H^{2}(n, 1)$ o espaço vetorial dos polinômios homogêneos de grau 2 em $n$ variáveis, ou seja, o espaço das formas quadráticas em $n$ variáveis. Este espaço sobre $\mathbb{K}$ possui dimensão $\left(\begin{array}{l}n \\ 2\end{array}\right)+n=\frac{n(n+1)}{2}$. Quando $n=2$, uma forma quadrática pode ser escrita como $a x^{2}+2 b x y+c y^{2}$ e assim pode ser identificada com o ponto $(a, b, c) \in \mathbb{K}^{3}$, ou ainda com a matriz simétrica $\left(\begin{array}{ll}a & b \\ b & c\end{array}\right)$. Mais geralmente temos:

Proposição 2.1.4. Há um isomorfismo natural entre $H^{2}(n, 1)$ e o espaço das matrizes simétricas $n \times n$.

Demonstração. Denotando as variáveis de um elemento em $H^{2}(n, 1)$ por $x_{1}, \ldots, x_{n}$, basta definirmos:

$$
\begin{aligned}
T: \operatorname{Sim}_{n}(\mathbb{K}) & \longrightarrow H^{2}(n, 1) \\
A & \longmapsto x^{t} A x,
\end{aligned}
$$

onde $x=\left(x_{1}, \ldots, x_{n}\right)$. 
Definição 2.1.5. Seja $\Gamma=\left\{\alpha: \mathbb{K}^{n} \rightarrow \mathbb{K}^{n} / \alpha\right.$ é uma transformação linear invertível $\}$. Temos a seguinte ação do grupo $\Gamma$ em $H^{2}(n, 1)$ :

$$
\begin{aligned}
\Phi: \Gamma \times H^{2}(n, 1) & \longrightarrow H^{2}(n, 1) \\
(\alpha, p) & \longmapsto p \circ \alpha^{-1} .
\end{aligned}
$$

Dizemos que duas quadráticas $p, q$ são $\Gamma$-equivalentes quando pertencem à mesma órbita por esta $a c ̧ \tilde{a} O$.

Observação 2.1.6. Esta ação possui formas normais bem conhecidas da álgebra linear. Quando $\mathbb{K}=\mathbb{C}$, podemos tomar as quadráticas $\sum_{i=1}^{r} x_{i}^{2}$, e em $\mathbb{R}$ temos $\sum_{i=1}^{s} x_{i}^{2}-\sum_{i=s+1}^{r} x_{i}^{2}$.

A ação $\Phi$ é compatível com a ação de $G L_{n}(\mathbb{K})$ em $\operatorname{Sim}_{n}(\mathbb{K})$ no seguinte sentido:

Proposição 2.1.7. Duas quadráticas são $\Gamma$-equivalentes se, e somente se, as suas matrizes correspondentes, pelo isomorfismo $T$, são $G L_{n}(\mathbb{K})$-equivalentes.

Com a equivalência da Proposição 2.1.7, podemos reduzir a classificação de 1-jatos de matrizes à classificação de pencils de formas quadráticas.

Às vezes, vamos olhar um germe $A:\left(\mathbb{K}^{r}, 0\right) \rightarrow \operatorname{Sim}_{n}(\mathbb{K})$, como um germe $A:\left(\mathbb{K}^{r}, 0\right) \rightarrow$ $\mathbb{K}^{N}$, onde $N=\frac{n(n+1)}{2}$. Assim, será útil o seguinte resultado:

Proposição 2.1.8. Existe um homomorfismo de grupos entre $G L_{n}(\mathbb{K})$ e $G L\left(\operatorname{Sim}_{n}(\mathbb{K})\right)=$ $G L_{N}(\mathbb{K})$.

A seguir, apresentamos alguns resultados gerais para espaços vetoriais, que nos fornecem uma importante dualidade e nos permitem simplificar alguns casos de nossa classificação de 1-jatos.

Definição 2.1.9. Sejam $V$ e $W$ espaços vetoriais de dimensão finita e $H$ um subgrupo de $G L(W)$. Dizemos que $\alpha_{1}, \alpha_{2} \in \operatorname{Hom}(V, W)$ são $G L(V) \times H$-equivalentes quando existem $\beta \in G L(V)$ e $\gamma \in H$, tais que:

$$
\alpha_{2}=\gamma \circ \alpha_{1} \circ \beta
$$

Definição 2.1.10. Se $\alpha \in \operatorname{Hom}(V, W)$ nós escrevemos $\alpha^{*}$ para o correspondente elemento de $\operatorname{Hom}\left(W^{*}, V^{*}\right)$.

Definição 2.1.11. Seja $U$ um subespaço de um espaço vetorial $V$. Escrevemos $U^{\perp}$ para $\left\{\phi \in V^{*} ; \phi(U)=0\right\}$.

Lema 2.1.12. (1) As aplicações $\alpha_{1}, \alpha_{2}$ são equivalentes se, e somente se, existe $\gamma \in H$ que leva $\operatorname{im} \alpha_{1}$ em $\operatorname{im} \alpha_{2}$.

(2) $\operatorname{Temos}(\operatorname{im} \alpha)^{\perp}=\operatorname{ker} \alpha^{*},(\operatorname{ker} \alpha)^{\perp}=\operatorname{im} \alpha^{*}$.

(3) As aplicações $\alpha_{1}, \alpha_{2}$ são equivalentes se, e somente se, para algum $\gamma \in H$ a aplicação $\gamma^{*}$ leva ker $\alpha_{1}^{*}$ em $\operatorname{ker} \alpha_{2}^{*}$. 
Aplicando o Lema 2.1.12 para $V=\mathbb{K}^{r}, W=\operatorname{Sim}_{n}(\mathbb{K})$ e $H=G L_{n}(\mathbb{K})$ agindo como um subgrupo de $G L\left(\operatorname{Sim}_{n}(\mathbb{K})\right)$ de maneira óbvia, segue pelo item (1), que classificar aplicações de posto $k$ em $\operatorname{Hom}\left(\mathbb{K}^{r}, \operatorname{Sim}_{n}(\mathbb{K})\right)$ pela $G L_{r}(\mathbb{K}) \times H$-equivalência é equivalente a classificar subespaços $k$-dimensionais de $\operatorname{Sim}_{n}(\mathbb{K})$ pela $H$-equivalência. Pelos itens (2) e (3), classificar

aplicações de posto $k$ é equivalente a classificar subespaços $\left(\frac{n(n+1)}{2}-k\right)$-dimensionais de $\left(\operatorname{Sim}_{n}(\mathbb{K})\right)^{*}$ pela $H$-equivalência. Isto nos fornece um resultado útil de dualidade que usaremos na classificação dos 1-jatos de matrizes.

Proposição 2.1.13. Há um isomorfismo linear natural $\operatorname{Sim}_{n}(\mathbb{K}) \rightarrow\left(\operatorname{Sim}_{n}(\mathbb{K})\right)^{*}$ definido por $A \mapsto \operatorname{tr}($ A.- $)$ identificando esses dois espaços.

Sendo assim, para a dualidade acima podemos usar o seguinte resultado que nos garante que classificar subespaços de dimensão $k$ em $\operatorname{Sim}_{n}(\mathbb{K})$ é o mesmo que classificar subespaços de dimensão $\frac{n(n+1)}{2}-1$ em $\operatorname{Sim}_{n}(\mathbb{K})$.

Proposição 2.1.14. Sejam $\left\{W_{i}\right\}, i=1,2, \ldots s$ subespaços de dimensão $k$ de Sim $_{n}$ equivalentes pela $G L_{n}(\mathbb{K})$-equivalência. Então os subespaços $\left\{W_{i}^{\perp}\right\}_{i=1}^{s}$, onde $W^{\perp}=\left\{A \in \operatorname{Sim}_{n} ; \operatorname{tr}(A B)=\right.$

0 para todo $B \in W\}$, é uma lista de subespaços de dimensão $\frac{n(n+1)}{2}-k$ de Sim $_{n}$ sobre a mesma equivalência.

\section{$2.2 \quad$ Grupo $\mathcal{G}$}

Denotamos por $\mathcal{S}$ o conjunto dos germes de aplicações suaves $A:\left(\mathbb{K}^{r}, 0\right) \rightarrow \operatorname{Sim}_{n}(\mathbb{K})$ e por $\mathcal{H}$ os germes de aplicações suaves $\left(\mathbb{K}^{r}, 0\right) \rightarrow G L_{n}(\mathbb{K})$. Usando a estrutura de grupo multiplicativo de $G L_{n}(\mathbb{K})$ podemos dar a $\mathcal{H}$ uma estrutura de grupo. Como já foi dito, será comum vermos um elemento de $\mathcal{S}$ como um germe de uma aplicação $\left(\mathbb{K}^{r}, 0\right) \rightarrow \mathbb{K}^{N}$, onde $N=\frac{n(n+1)}{2}$.

Usando os grupos $\mathcal{R}$ e $\mathcal{H}$ queremos definir uma ação de grupo em $\mathcal{S}$ que "preserve" certas propriedades. Mas antes, relembremos o conceito de produto semidireto de dois grupos.

Sejam $K$ e $H$ dois grupos (não necessariamente finitos), se $\sigma: K \rightarrow A u t(H)$ é um homomorfismo do grupo $K$ no grupo dos automorfismos de $H$ então $\dot{\sigma}$ denotará a operação definida sobre o conjunto $K \times H$ da seguinte maneira:

$$
(k, h)_{\dot{\sigma}}\left(k^{\prime}, h^{\prime}\right)=\left(k \cdot k^{\prime}, h \cdot \sigma(k)\left(h^{\prime}\right)\right) .
$$

Teorema 2.2.1. Seja $K, H$ dois grupos e $\sigma: K \rightarrow$ Aut $(H)$ um homomorfismo. Então $(K \times H, \dot{\sigma})$ é um grupo.

Definição 2.2.2. O grupo $(K \times H, \dot{\sigma})$ é chamado de produto semidireto de $H$ por $K$ com homomorfismo $\sigma$. 
Passemos a definir uma estrutura de grupo em $\mathcal{R} \times \mathcal{H}$. Denotemos por $\operatorname{Aut}(\mathcal{H})$ o grupo de automorfismos de $\mathcal{H}$ e definimos o seguinte homomorfismo de grupos:

$$
\begin{aligned}
& \sigma: \mathcal{R} \longrightarrow \operatorname{Aut}(\mathcal{H}) \\
& \phi \longmapsto \sigma(\phi): \mathcal{H} \rightarrow \mathcal{H} \\
& X \mapsto X \circ \phi^{-1} \text {, assim temos: }
\end{aligned}
$$

Definição 2.2.3. O grupo $\mathcal{G}$ é o produto semidireto de $\mathcal{R}$ por $\mathcal{H}$ com homomorfismo $\sigma$.

Definição 2.2.4. Dois germes suaves $A, B \in \mathcal{S}$ são $\mathcal{G}$-equivalentes se existe $(\phi, X) \in \mathcal{G}$ tal que:

$$
B=X^{t}\left(A \circ \phi^{-1}\right) X
$$

Esta equivalência claramente nos fornece uma ação de $\mathcal{G}$ em $\mathcal{S}$.

Observação 2.2.5. Pela Proposição 2.1.8, temos um homomorfismo de grupos entre o grupo $G L_{n}(\mathbb{K})$ e o grupo $G L\left(\operatorname{Sim}_{n}\right)=G L_{N}(\mathbb{K})$, que denotaremos por $\xi$. Este homomorfismo nos fornece um homomorfismo de grupos entre o grupo $\mathcal{H}$ e o grupo $\mathcal{C}$. De fato, lembrando que as estruturas de grupo de $\mathcal{H}$ e $\mathcal{C}$, provém das estruturas de grupo de $G L_{n}(\mathbb{K})$ e $G L_{N}(\mathbb{K})$, basta definirmos este homomorfismo por:

$$
\begin{aligned}
\vartheta: \mathcal{H} & \rightarrow \mathcal{C} \\
X & \rightarrow \xi \circ X .
\end{aligned}
$$

Lema 2.2.6. O grupo $\mathcal{G}=\mathcal{R} \times \mathcal{H}$ age no espaço das aplicações $\left(\mathbb{K}^{r}, 0\right) \rightarrow \mathbb{K}^{N}$ como um subgrupo do correspondente grupo de contato $\mathcal{K}$.

Demonstração. A ação do grupo $\mathcal{R}$ em ambos os casos claramente coincidem. Pela Observação 2.2.5, temos um homomorfismo entre $\mathcal{H}$ e $\mathcal{C}$. Logo, dado $X \in \mathcal{H}$ podemos correspondê-lo a um elemento $\tilde{X} \in \mathcal{C}$, que age linearmente em $\mathbb{K}^{N}$ da mesma forma que $X$ age em $\operatorname{Sim}_{n}(\mathbb{K})$. Em outras palavras, dada uma aplicação $A:\left(K^{r}, 0\right) \rightarrow\left(\mathbb{K}^{N}, 0\right)$ e um par $(\phi, X) \in \mathcal{R} \times \mathcal{H}$, a ação deste par na aplicação $A$ olhando um elemento de $\mathbb{K}^{N}$ como um elemento de $\operatorname{Sim}_{n}(\mathbb{K})$ coincide com a ação do $\operatorname{par}(\phi, \widetilde{X}) \in \mathcal{R} \times \mathcal{C}=\mathcal{K}$ em $A$, e o resultado segue.

Exemplifiquemos a demonstração acima para $r=n=2$.

Exemplo 2.2.7. Dado um elemento $X \in \mathcal{H}$, ele atua nas aplicações $A:\left(\mathbb{K}^{2}, 0\right) \rightarrow\left(\operatorname{Sim}_{2}(\mathbb{K}), 0\right)$, da mesma forma que o elemento $\vartheta(X)=\widetilde{X} \in \mathcal{C}$ atua nas aplicações $\widetilde{A}:\left(\mathbb{K}^{2}, 0\right) \rightarrow\left(\mathbb{K}^{3}, 0\right)$ onde :

$$
X=\left(\begin{array}{cc}
\alpha & \beta \\
\gamma & \delta
\end{array}\right) \quad \text { e } \quad \tilde{X}=\left(\begin{array}{ccc}
\alpha^{2} & 2 \alpha \gamma & \gamma^{2} \\
\alpha \beta & \alpha \delta+\beta \gamma & \gamma \delta \\
\beta^{2} & 2 \beta \delta & \delta^{2}
\end{array}\right)
$$

Mais explicitamente, dado $A:\left(\mathbb{K}^{2}, 0\right) \rightarrow\left(\operatorname{Sim}_{2}(\mathbb{K}), 0\right)$, podemos identificá-la trivialmente a uma aplicação $\widetilde{A}:\left(\mathbb{K}^{2}, 0\right) \rightarrow\left(\mathbb{K}^{3}, 0\right)$, isto é, se $\phi=\left(\begin{array}{ll}a & b \\ b & c\end{array}\right)$ então $\widetilde{A}=(a, b, c)$. Logo, 
$X^{t} A X$ será uma aplicação de $\left(\mathbb{K}^{2}, 0\right) \rightarrow$ Sim $_{2}(\mathbb{K})$ e se a identificarmos com uma aplicação $\left(\mathbb{K}^{2}, 0\right) \rightarrow\left(\mathbb{K}^{3}, 0\right)$, como acima, obtemos uma aplicação de $\left(\mathbb{K}^{2}, 0\right) \rightarrow\left(\mathbb{K}^{3}, 0\right)$ que será igual a $\widetilde{X} \widetilde{A}$.

O fato que nos permite usar todas as técnicas da teoria de singularidades para o grupo $\mathcal{G}$ segue do fato que $\mathcal{G}$ é um subgrupo geométrico de Damon do grupo de contato $\mathcal{K}$. Para a definição de subgrupo geométrico, ver [9].

Exemplificamos abaixo algumas estruturas preservadas pela $\mathcal{G}$-equivalência.

Definição 2.2.8. O discriminante de um elemento $A \in \mathcal{S}$ é o germe do conjunto:

$$
D(A), 0=\left\{x \in \mathbb{K}^{r} ; \operatorname{det}(A(x))=0\right\}, 0 .
$$

O próximo resultado nos diz que o tipo de singularidade deste elemento é preservada pela $\mathcal{G}$-equivalência.

Proposição 2.2.9. Se $A, B \in \mathcal{S}$ são $\mathcal{G}$-equivalentes então existe um germe de difeomorfismo $\phi:\left(\mathbb{K}^{r}, 0\right) \rightarrow\left(\mathbb{K}^{r}, 0\right)$, preservando o discriminante, isto é, levando $D(A)$ em $D(B)$.

Vimos na introdução, que uma motivação para o estudo de germes $\left(\mathbb{K}^{r}, 0\right) \rightarrow \operatorname{Sim}_{n}(\mathbb{K})$ é a sua relação com equações diferenciais binárias (EDB's). Lembremos que uma EDB é uma equação diferencial da forma

$$
a(x, y) d y^{2}+2 b(x, y) d x d y+c(x, y) d x^{2}=0
$$

Dada uma EDB, podemos relacioná-la com a matriz

$$
\left(\begin{array}{ll}
a(x, y) & b(x, y) \\
b(x, y) & c(x, y)
\end{array}\right)
$$

a qual nós podemos pensar como uma aplicação $A:\left(\mathbb{R}^{2}, 0\right) \rightarrow \operatorname{Sim}_{2}(\mathbb{R})$, cujo determinante é o discriminante da EDB. Como vimos acima, $\mathcal{G}$ preserva as singularidades do determinante. O seguinte resultado relaciona o fato acima com o estudo das EDB's.

Proposição 2.2.10. Dada uma EDB $F=a(x, y) d y^{2}+2 b(x, y) d x d y+c(x, y) d x^{2}=0$, escrevemos $A(F)$ para o seu correspondente elemento de $\mathcal{S}$. Se $\phi:\left(\mathbb{R}^{2}, 0\right) \rightarrow\left(\mathbb{R}^{2}, 0\right)$ é o germe de um difeomorfismo levando a $E D B F=0$ em uma $E D B G=0$ então $A(F)$ e $A(G)$ são $\mathcal{G}$-equivalentes.

No Capítulo 4 do artigo [25], o autor usa a classificação de matrizes simétricas para obter resultados sobre as EDB's. 


\subsection{Espaço tangente}

Dada uma família de matrizes $A \in \mathcal{S}$, escreveremos $A_{x_{i}}$ para a matriz $\frac{\partial A}{\partial x_{i}}$. O conjunto $\mathcal{S}$ pode ser identificado com $\mathcal{O}(r, N)$, onde $N=\frac{n(n+1)}{2}$, e o grupo $\mathcal{G}$ com um subgrupo do correspondente grupo de contato $\mathcal{K}$, como vimos na seção anterior.

Na próxima proposição exibimos quem será o espaço tangente à órbita de um dado elemento $A$. Mas para isso precisamos de algumas definições. Definimos $C_{i j}(A)$ (respectivamente $R_{i j}(A)$ ) como sendo a matriz cuja i-ésima coluna (resp. linha) é a j-ésima coluna (resp. linha) de $A$ e nas outras posições igual a zero. A matriz $A_{i j}$ será a matriz dada por $A_{i j}=C_{i j}(A)+R_{i j}(A)$.

Exemplo 2.3.1. $A=\left(\begin{array}{cc}x & x+y \\ x+y & y\end{array}\right)$, temos que

$$
\begin{aligned}
& A_{11}=C_{11}(A)+R_{11}(A)=\left(\begin{array}{cc}
x & 0 \\
x+y & 0
\end{array}\right)+\left(\begin{array}{cc}
x & x+y \\
0 & 0
\end{array}\right)=\left(\begin{array}{cc}
2 x & x+y \\
x+y & 0
\end{array}\right) \\
& A_{12}=C_{12}(A)+R_{12}(A)=\left(\begin{array}{cc}
x+y & 0 \\
y & 0
\end{array}\right)+\left(\begin{array}{cc}
x+y & y \\
0 & 0
\end{array}\right)=\left(\begin{array}{cc}
2(x+y) & y \\
y & 0
\end{array}\right) \\
& A_{21}=C_{21}(A)+R_{21}(A)=\left(\begin{array}{cc}
0 & x \\
0 & x+y
\end{array}\right)+\left(\begin{array}{cc}
0 & 0 \\
x & x+y
\end{array}\right)=\left(\begin{array}{cc}
0 & x \\
x & 2(x+y)
\end{array}\right) \\
& A_{22}=C_{22}(A)+R_{22}(A)=\left(\begin{array}{cc}
0 & x+y \\
0 & y
\end{array}\right)+\left(\begin{array}{cc}
x+y & 0
\end{array}\right)=\left(\begin{array}{cc}
0 & x+y \\
x+y & 2 y
\end{array}\right)
\end{aligned}
$$

\section{Proposição 2.3.2 (Espaço Tangente).}

(1) $O \mathcal{R}$-espaço tangente à órbita de um elemento $A \in \mathcal{S}$ é o $\mathcal{O}$-módulo gerado por $x_{j} A_{x_{i}}$, com $1 \leqslant i, j \leqslant r$.

(2) O espaço tangente a órbita de $A$ sobre o grupo $\mathcal{H}$ de $\mathcal{G}$ é o $\mathcal{O}$-módulo gerado por $A_{i j}=$ $C_{i j}(A)+R_{i j}(A)$, com $1 \leqslant i, j \leqslant n$. Portanto:

$$
T \mathcal{G} A=\mathcal{M}\left\{A_{x_{i}}, 1 \leqslant i \leqslant r\right\}+\mathcal{O}\left\{A_{i j}, 1 \leqslant i, j \leqslant n\right\}
$$

Observação 2.3.3. A dimensão do espaço tangente à órbita de um elemento em $J^{1} \mathcal{S}$ pela ação do grupo $J^{1} \mathcal{G}$ será no máximo $r^{2}+n^{2}-1$. Com efeito, podemos ver que $2 \sum_{i=1}^{r} x_{i} A_{x_{i}}=$ $\sum_{i=1}^{n} A_{i i}$, ou seja, existe pelo menos um gerador linearmente dependente. Portanto, sua dimensão pode ser no máximo $r^{2}+n^{2}-1$. 
Definição 2.3.4. O espaço tangente estendido para o grupo $\mathcal{G}$ aplicado ao germe $A$, denotado por $T_{e} \mathcal{G} A$ é o $\mathcal{O}$-módulo gerado por $A_{x_{i}}$ e $A_{i j}$.

Nos artigos [10] e [11], James Damon introduz a $\mathcal{K}_{V}$-equivalência, onde $V$ é uma variedade analítica real ou complexa. No Apêndice C, alguns resultados sobre esta teoria foram apresentados para que o leitor tenha acesso direto às idéias aqui comentadas.

Relacionaremos nossa equivalência com a $\mathcal{K}_{V}$-equivalência. Considerando a variedade $V=\Delta$, como sendo o conjunto das matrizes simétricas singulares e o ideal dos germes que se anulam em $V$, denotado por $I(V)=I(\Delta)$, que é gerado pelo germe:

$$
\text { det: } \begin{aligned}
\left(\operatorname{Sim}_{n}, 0\right) & \rightarrow \\
A & \rightarrow \operatorname{det}(A)
\end{aligned}
$$

Definição 2.3.5. Denotamos por $V \mathcal{H}$ os campos de vetores em $\operatorname{Sim}_{n}(\mathbb{C})$ determinado pelo grupo $\mathcal{H}$, ou seja, $V \mathcal{H}$ é o $\mathcal{O}_{N}$-módulo gerado por $A_{i j}$.

Observação 2.3.6. Cada $A_{i j}$ pode ser visto como um campo de vetores dado por

$$
A_{i j}=2 \sum_{k=1}^{n}\left(\frac{1}{2-\delta_{i k}}\right) a_{k j} \frac{\partial}{\partial a_{k i}}
$$

Proposição 2.3.7. O módulo do campo de vetores holomórficos tangentes ao discriminante $\Delta$, que denotamos por Derlog $(\Delta)$ (ver Apêndice $C$ ), coincide com $V \mathcal{H}$.

Demonstração. Claramente $V \mathcal{H} \subset \operatorname{Derlog}(\Delta)$, pois $\mathcal{H}$ preserva $\Delta$. Por outro lado, dado um campo de vetores $\theta=\sum \alpha_{i j} \partial / \partial a_{i j} \in \operatorname{Derlog}(\Delta)$, devemos ter $\theta($ det $) \in I(\Delta)$, ou seja, $\theta($ det $)=$ $\alpha$ det, para alguma função analítica $\alpha$. Temos que o campo de Euler pertence a ambos os módulos. Subtraindo um múltiplo dele de $\theta$ podemos considerar $\theta($ det $)=0$, ou seja,

$$
\sum_{i, j=1}^{n} \alpha_{i j} \frac{\partial d e t}{\partial a_{i j}}=\sum_{i, j=1}^{n} \alpha_{i j}\left(2-\delta_{i j}\right)(-1)^{i+j} M_{i j}=0
$$

onde $M_{i j}$ é o menor complementar do elemento $a_{i j}$ e $\delta_{i j}$ é o delta de Kronecker.

Em [18], temos que as relações entre os menores complementares são geradas por:

$$
\sum_{i=1}^{n}(-1)^{i+k} a_{i j} M_{i k}=\delta_{j k} \operatorname{det}(A) \text { para } 1 \leqslant j, k \leqslant n,
$$

que são obtidas pela igualdade $A \cdot \operatorname{adj}(A)=\operatorname{det}(A) I$.

Segue fácil que $\sum_{i=1}^{n}\left(\frac{1}{2-\delta_{i k}}\right) a_{i j} \frac{\partial}{\partial a_{i k}}$ para $1 \leqslant j, k \leqslant n \operatorname{geram} \operatorname{Derlog}(\Delta)$. Mas, pela Observação 2.3.6 esses são nossos geradores para $V \mathcal{H}, \operatorname{logo} \operatorname{Derlog}(\Delta) \subset V \mathcal{H}$. Portanto, $\operatorname{Derlog}(\Delta)=V \mathcal{H}$

Proposição 2.3.8. Sobre $\mathbb{C}$ a noção de $\mathcal{K}_{\Delta}$ e $\mathcal{G}$-equivalência coincidem. 
Demonstração. O grupo $\mathcal{H}$ preserva o conjunto $\Delta$, assim $\mathcal{G} \subset \mathcal{R} \times \mathcal{C}_{\Delta}$. Mas pela Proposição 2.3.7 os espaços tangentes coincidem e resultado segue.

Pelo Lema 2.2.6, podemos ver o grupo $\mathcal{G}$ como um subgrupo do correspondente grupo contato $\mathcal{K}$. Assim, temos também o seguinte resultado para $\mathcal{G}$ :

Proposição 2.3.9 (Transversal Completa). Seja $A$ um $k$-jato em $J^{k} \mathcal{S}$, escrevemos $\mathcal{G}_{1}$ para o subgrupo de $\mathcal{G}$ dos elementos cujo 1-jato é a identidade, e $H^{k+1} \mathcal{S}$ para as aplicações polinomiais homogêneas de grau $k+1$. Suponha que $T_{A} J^{k+1} \mathcal{G}_{1} \cdot A \cap H^{k+1} \mathcal{S}+T=H^{k+1} \mathcal{S}$ para algum subespaço $T$ de $H^{k+1} \mathcal{S}$. Então todo $(k+1)$-jato com $k$-jato $A$ é $J^{k+1} \mathcal{G}_{1}$-equivalente a um jato da forma $A+B$, com $B \in T$. Tal $T$ é chamado de transversal completa.

Observação 2.3.10. Se $A \in \mathcal{S}$, temos que

$$
T \mathcal{G}_{1} \cdot A=\mathcal{M}^{2}\left\{A_{x_{i}}, 1 \leqslant i \leqslant r\right\}+\mathcal{M}\left\{A_{i j}, 1 \leqslant i, j \leqslant n\right\}
$$

\subsection{Determinação Finita}

Nessa seção exibimos condições necessárias e suficientes para que um germe seja finitamente determinado e alguns critérios para verificar seu grau de determinação.

Vimos na seção anterior que a noção de $\mathcal{K}_{V}$ e $\mathcal{G}$-equivalência coincidem sobre $\mathbb{C}$. Assim, alguns resultados que estão feitos para $\mathcal{K}_{V}$, que podem ser encontrados em [10] e [11], serão assumidos. Boa parte dos resultados obtidos para $\mathcal{G}\left(\right.$ ou $\left.\mathcal{K}_{V}\right)$, sobre determinação finita, seguem análogo aos resultados encontrados em [26] para os grupos $\mathcal{R}, \mathcal{L}, \mathcal{A}, \mathcal{C}$ e $\mathcal{K}$. Apresentamos também nessa seção uma caracterização geométrica dos germes $\mathcal{G}$-finitamente determinados.

Comecemos com algumas definições. Dado $A:\left(\mathbb{K}^{r}, 0\right) \rightarrow \operatorname{Sim}_{n}(\mathbb{K})$, definimos a $\mathcal{G}_{\text {- }}$ codimensão de $A$ como

$$
\mathcal{G}-\operatorname{cod}(A)=\operatorname{dim}_{\mathbb{K}}\left(\mathcal{M}_{r} \mathcal{S} / T \mathcal{G} \cdot A\right)
$$

e a $\mathcal{G}_{e^{-} \text {-codimensão de } A \text { como }}$

$$
\mathcal{G}_{e}-\operatorname{cod}(A)=\operatorname{dim}_{\mathbb{K}}\left(\mathcal{S} / T_{e} \mathcal{G} \cdot A\right), \text { assim: }
$$

Proposição 2.4.1. São equivalentes:

(i) $\mathcal{G}-\operatorname{cod}(A)<\infty$

(ii) $\mathcal{G}_{e}-\operatorname{cod}(A)<\infty$

(iii) Existe l tal que $\mathcal{M}^{l} \mathcal{S} \subset T \mathcal{G} A$

(iv) Existe $k$ tal que $\mathcal{M}^{k} \mathcal{S} \subset T_{e} \mathcal{G} A$ 
Demonstração. As equivalências $(i) \Leftrightarrow(i i i)$ e $(i i) \Leftrightarrow(i v)$, seguem direto da Proposição 1.1.20. Logo, resta mostrarmos $(i) \Leftrightarrow(i i)$.

$(i) \Rightarrow(i i)$; Como $T \mathcal{G} A \subset T_{e} \mathcal{G} A$, segue que se $T \mathcal{G} A$ tem codimensão finita então $T_{e} \mathcal{G} A$ tem codimensão finita.

$($ ii $) \Rightarrow(i)$; Por hipótese $\mathcal{G}_{e}-\operatorname{cod} A<\infty, \operatorname{logo} \exists k$ tal que $\mathcal{M}^{k} \mathcal{S} \subset T_{e} \mathcal{G} A$, ou seja, $\mathcal{M}^{k} \mathcal{S} \subset$ $\mathcal{O}\left\{A_{x_{i}}, A_{i j}\right\}$. Mas, $\mathcal{M}^{k} \mathcal{S} \subset \mathcal{O}\left\{A_{x_{i}}, A_{i j}\right\} \Rightarrow \mathcal{M}^{k+1} \mathcal{S} \subset \mathcal{M}\left\{A_{x_{i}}, A_{i j}\right\} \subset \mathcal{O}\left\{\mathcal{M} A_{x_{i}}, A_{i j}\right\}=T \mathcal{G} . A$, ou seja, $\mathcal{G}$-cod $A<\infty$.

As duas próximas proposições exibem critérios para que possamos verificar se um determinado germe é finitamente determinado.

Proposição 2.4.2. Se $A:\left(\mathbb{K}^{r}, 0\right) \rightarrow \operatorname{Sim}_{n}(\mathbb{K})$ é $k$-G-determinado, então $\mathcal{M}^{k+1} \mathcal{S} \subset T \mathcal{G} A$.

Demonstração. Como $A$ é $k$-determinado, sem perda de generalidade podemos supor que $A \in J^{k} \mathcal{S}$. Consideremos a seguinte projeção linear:

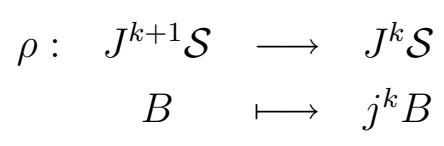

Como $\rho$ é linear e sobrejetora, segue que $\rho$ é uma submersão e assim $\rho^{-1}(A)$ é uma subvariedade em $J^{k+1} \mathcal{S}$. Por [20], temos que o espaço tangente de $\rho^{-1}(A)$ é o núcleo de $\rho$, ou seja, $\mathcal{M}^{k+1} \mathcal{S} / \mathcal{M}^{k+2} \mathcal{S} \cong$ ao espaço vetorial das aplicações polinomiais homogêneas de grau $k+1$.

Afirmação: $\rho^{-1}(A) \subset J^{k+1} \mathcal{G} \cdot A$.

De fato, dado $B \in \rho^{-1}(A)$ temos que $j^{k} B=A$. Como $A$ é $k$-determinado segue que $B$ é $\mathcal{G}$-equivalente a $A$. Assim existe $(\phi, X) \in \mathcal{G}$ tal que $B=X^{t}\left(A \circ \phi^{-1}\right) X$. Logo, $j^{k+1} B=B=$ $j^{k+1}\left[X^{t}\left(A \circ \phi^{-1}\right) X\right] \in J^{k+1} \mathcal{G} . A$. Portanto $\rho^{-1}(A) \subset J^{k+1} \mathcal{G} . A$.

Logo, a menos de identificações, segue que

$$
\begin{gathered}
\mathcal{M}^{k+1} \mathcal{S} / \mathcal{M}^{k+2} \mathcal{S}=T\left(\rho^{-1}(A)\right) \subset T J^{k+1} \mathcal{G} . A=\left(T \mathcal{G} . A+\mathcal{M}^{k+2} \mathcal{S}\right) / \mathcal{M}^{k+2} \mathcal{S} \\
\Rightarrow \mathcal{M}^{k+1} \mathcal{S} \subset T \mathcal{G} \cdot A+\mathcal{M}^{k+2} \mathcal{S}
\end{gathered}
$$

Usando o Lema de Nakayama 1.1 .19 obtemos $\mathcal{M}^{k+1} \mathcal{S} \subset T \mathcal{G} . A$

Proposição 2.4.3. Se $\mathcal{M}^{k} \mathcal{S} \subset T \mathcal{G} . A$ então A é k-determinado.

Demonstração. Ver ([10], p. 708).

Usando as proposições acima, podemos obter:

Proposição 2.4.4. Uma condição necessária e suficiente para que um germe $A \in \mathcal{S}$ seja finitamente determinado é que $\mathcal{M}^{k} \mathcal{S} \subset T_{e} \mathcal{G} . A$, para algum $k \in \mathbb{N}$. 
Passemos a trabalhar sobre $\mathbb{C}$. A Proposição 2.1.1 nos diz que a ação de $G L_{n}(\mathbb{C})$ sobre $\operatorname{Sim}_{n}(\mathbb{C})$ possui $n+1$ órbitas. Estas órbitas determinam uma estratificação de $\operatorname{Sim}_{n}(\mathbb{C})$. Dizemos que um germe $A:\left(\mathbb{C}^{r}, 0\right) \rightarrow \operatorname{Sim}_{n}(\mathbb{C})$ é transversal à estratificação de $\operatorname{Sim}_{n}(\mathbb{C})$ em um ponto $z_{0} \in \mathbb{C}^{r}$ quando $A_{z_{0}}^{\prime}\left(\mathbb{C}^{r}\right)+T_{A\left(z_{0}\right)}\left(G L_{n} \cdot A\left(z_{0}\right)\right)=\operatorname{Sim}_{n}(\mathbb{C})$, ou seja, quando o $\mathbb{C}$-espaço vetorial gerado por $\left\{A_{x_{i}}\left(z_{0}\right), A_{i j}\left(z_{0}\right)\right\}$ for todo $\operatorname{Sim}_{n}(\mathbb{C})$.

Observação 2.4.5. Quando $A\left(z_{0}\right)$ for uma matriz invertível segue da Observação 2.1.3 que o $\mathbb{C}$-espaço vetorial gerado por $A_{i j}\left(z_{0}\right)$ é todo $\operatorname{Sim}_{n}(\mathbb{C})$. Portanto, para todo $z_{0} \in \mathbb{C}^{r}$ em que $A\left(z_{0}\right)$ for invertivel temos que A é transversal à estratificação de $\operatorname{Sim}_{n}(\mathbb{C})$ em $z_{0}$.

Assim como para os grupos de Mather, também temos uma caracterização geométrica para germes $\mathcal{G}$-finitamente determinados que será enunciada na próxima proposição. Em [10], James Damon exibi uma prova deste resultado para o grupo $\mathcal{K}_{V}$ que é praticamente análoga à prova dada por Mather para o grupo $\mathcal{K}$, com suas devidas modificações. Fornecemos esta prova no Apêndice A para o grupo $\mathcal{G}$. Em [5], J.W.Bruce fornece uma prova deste mesmo resultado usando a teoria de feixes, que segue os argumentos utilizados por Gaffney. Para maiores detalhes desta prova, usando feixes, ver [12] ou ([26], p. 492).

Proposição 2.4.6. Um elemento $A \in \mathcal{S}$ é $\mathcal{G}$-finitamente determinado se, e somente se, $A$ é transversal à estratificação de $\operatorname{Sim}_{n}(\mathbb{C})$ fora da origem.

Corolário 2.4.7. Seja $A \in \mathcal{S}$ um germe $\mathcal{G}$-finitamente determinado. Então não existe pontos $x$ em uma vizinhança da origem no qual a matriz $A(x)$ possui coposto $s$, onde $r \leqslant \frac{s(s+1)}{2}$.

Demonstração. O conjunto das matrizes simétricas de coposto $s$ é uma variedade diferenciável de codimensão $\frac{s(s+1)}{2}$ em $\operatorname{Sim}_{n}(\mathbb{C})$. Dado $A:\left(\mathbb{C}^{r}, 0\right) \rightarrow \operatorname{Sim}_{n}(\mathbb{C})$, um germe $\mathcal{G}$-finitamente determinado, temos pela Proposição 2.4.6 que ele é transversal às órbitas da estratificação de $\operatorname{Sim}_{n}(\mathbb{C})$ fora da origem. Assim, em uma vizinhança furada da origem a imagem inversa do conjunto das matrizes de coposto $s$ é uma variedade suave de codimensão $\frac{s(s+1)}{2}$, ou seja, de dimensão $r-\frac{s(s+1)}{2}$. Portanto é vazia se $r \leqslant \frac{s(s+1)}{2}$.

Definição 2.4.8. Dizemos que um elemento $A \in \mathcal{S}$ é $\mathcal{G}$-estável quando $T_{e} \mathcal{G} A=\mathcal{S}$.

Proposição 2.4.9. Um elemento $A \in \mathcal{S}$ é $\mathcal{G}$-estável se, e somente se, os vetores $A_{x(i)}(0)$ são transversais à órbita de $G L_{n}(\mathbb{C})$ em $A(0)$.

Demonstração. Suponhamos que $A$ é $\mathcal{G}$-estável. Dado $B \in \operatorname{Sim}_{n}(\mathbb{C})$, consideremos o germe constante $W:\left(\mathbb{C}^{r}, 0\right) \rightarrow \operatorname{Sim}_{n}(\mathbb{C}), \operatorname{com} W(x)=B, \forall x \in \mathbb{C}^{r}$. Como $A$ é $\mathcal{G}$-estável existem $f_{1}, \ldots, f_{r}, g_{11}, \ldots, g_{n n} \in \mathcal{O}$ tais que

$$
W=\sum_{j} f_{j} A_{x_{j}}+\sum_{k, l} g_{k l} A_{k l} .
$$


Aplicando na origem, temos

$$
B=\sum_{j} f_{j}(0) A_{x_{j}}(0)+\underbrace{\sum_{k, l} g_{k l}(0) A_{k l}(0)}_{\in T_{A(0)}(G L \cdot A(0))} .
$$

Como $B$ é arbitrário, segue que os vetores $A_{x_{j}}(0)$ são transversais à órbita de $G L_{n}(\mathbb{C})$ em $A(0)$.

Por outro lado, se os vetores $A_{x_{j}}(0)$ são transversais à órbita de $G L_{n}(\mathbb{C})$ em $A(0)$ então

$$
\mathcal{S} \subset T_{e} \mathcal{G} \cdot A+\mathcal{M S}
$$

Aplicando o Lema de Nakayma 1.1.19, segue que $\mathcal{S} \subset T_{e} \mathcal{G} . A$ e portanto $A$ é $\mathcal{G}$-estável.

Um resultado padrão na teoria de singularidades é que quase todas as aplicações são $\mathcal{K}$ finitamente determinadas, ver ([26], p. 513). Com uma pequena variação na prova desse resultado podemos obter um resultado análogo para o grupo $\mathcal{G}$. Mas antes, necessitamos de algumas definições e resultados.

Sejam $V_{k} \subset J^{k} \mathcal{S}$ subconjuntos algébricos, com $V_{k+1} \subset \rho^{-1}\left(V_{k}\right)$. O conjunto $\mathrm{V}$ dos germes de aplicações $f$ com $j^{k} f \in V_{k}$, para todo $k$, é chamado de conjunto proalgébrico. Claramente, $\operatorname{cod} V_{k} \leqslant \operatorname{cod} V_{k+1}$. Escreveremos $\operatorname{cod} V$ para o limite de $\operatorname{cod} V_{K}$, quando $k \rightarrow \infty$. Uma propriedade de germes de aplicações que é válida exceto em um conjunto proalgébrico de codimensão infinita é chamada de propriedade geral.

Proposição 2.4.10. Seja $A$ um conjunto proalgébrico, temos que cod $V=\infty$ se, e somente se, para cada $z \in J^{k} \mathcal{S}$ nós podemos exibir $f \notin V$ com $j^{k} f=z$.

Demonstração. Ver ([26], p. 513).

Na página 513 de [26], mostra-se que o conjunto de germes que não são $\mathcal{K}$-finitamente determinados é um conjunto proalgébrico. Analogamente podemos mostrar que conjunto de germes que não são $\mathcal{G}$-finitamente determinados é um conjunto proalgébrico. Logo, obtemos o seguinte resultado:

Proposição 2.4.11. Para todo $r, n$ os germes $A:\left(\mathbb{C}^{r}, 0\right) \rightarrow \operatorname{Sim}_{n}(\mathbb{C})$ que não têm $\mathcal{G}$ codimensão finita formam um conjunto de codimensão infinita em $\mathcal{S}$.

Demonstração. Usando a Proposição 2.4.10 é suficiente mostrarmos que para todo germe de aplicações polinomiais de grau $m, A:\left(\mathbb{C}^{r}, 0\right) \rightarrow \operatorname{Sim}_{n}(\mathbb{C})=\mathbb{C}^{N}$, podemos exibir uma aplicação polinomial $B:\left(\mathbb{C}^{r}, 0\right) \rightarrow \operatorname{Sim}_{n}(\mathbb{C})$ de grau $m+1 \operatorname{com} j^{m} B=A$ e $B \operatorname{com} \mathcal{G}$ codimensão finita.

Seja $H^{m+1}$ o espaço das aplicações polinomiais homogêneas $\left(\mathbb{C}^{r}, 0\right) \rightarrow \operatorname{Sim}_{n}(\mathbb{C})$ de grau $m+1$ e considere a aplicação $G: \mathbb{C}^{r} \times H^{m+1} \rightarrow \operatorname{Sim}_{n}(\mathbb{C})$, definida por $G(x, C)=A(x)+C(x)$. 
Temos que $G$ é uma submersão quando restrita a $\left(\mathbb{C}^{r}-\{0\}\right) \times H^{m+1}$ e em particular $G$ : $\left(\mathbb{C}^{r}-\{0\}\right) \times H^{m+1} \rightarrow \operatorname{Sim}_{n}(\mathbb{C})$ é transversal à $\Delta$. O Lema Básico de Transversalidade de Thom (ver [13]), assegura que para quase todo $C \in H^{m+1}$ nós temos $G(-, C):\left(\mathbb{C}^{r}-\{0\}\right) \rightarrow \operatorname{Sim}_{n}(\mathbb{C})$ transversal à $\Delta$. Seja $C$ tal que $G(-, C):\left(\mathbb{C}^{r}-\{0\}\right) \rightarrow \operatorname{Sim}_{n}(\mathbb{C})$ é transversal à $\Delta$ e defina $B=A+C$. Claramente temos que $j^{m} B=A$ e por construção $B$ é transversal à $\Delta$ fora da origem. Pela Proposição 2.4.6 temos que $B$ é $\mathcal{G}$-finitamente determinado e o resultado segue.

\subsection{Germes Simples}

Estamos interessados na classificação de germes simples de aplicações $A:\left(\mathbb{C}^{r}, 0\right) \rightarrow$ $\operatorname{Sim}_{n}(\mathbb{C})$. Nesta seção, vamos definir germes simples e apresentar critérios que os identifiquem. Em seguida vamos discutir quais são os possíveis valores de $r, n$ para que possamos obter germes simples. Entre esses valores, aparecerão $n=2$ e $n=3$, para os quais identificamos na próxima seção com pencils de quadráticas em 2 e 3 variáveis, respectivamente.

Definição 2.5.1. Um germe finitamente determinado $f \in \mathcal{M}_{n} \mathcal{O}(n, p)$ é dito $\mathcal{G}$-simples se existe vizinhança $V$ de $j^{k} f$ (k grande) em $J^{k}(n, p)$ tal que $V$ encontra um número finito de $J^{k} G$-órbitas.

Observação 2.5.2. Se $f \in \mathcal{M}_{n} \mathcal{O}(n, p)$ é simples então $j^{k} f(0)$ é simples para qualquer $k \geqslant 1$.

Dadas aplicações $A_{j}:\left(\mathbb{K}^{r}, 0\right) \rightarrow \operatorname{Sim}_{n(j)}, j=1,2$, denotaremos por $A_{1} \oplus A_{2}:\left(\mathbb{K}^{r}, 0\right) \rightarrow$ $\operatorname{Sim}_{n(1)+n(2)}$ a aplicação dada por:

$$
(x, y) \rightarrow\left(\begin{array}{cc}
A_{1}(x, y) & 0 \\
0 & A_{2}(x, y)
\end{array}\right)
$$

Proposição 2.5.3. Dado um germe $A:\left(\mathbb{K}^{r}, 0\right) \rightarrow \operatorname{Sim}_{n}(\mathbb{K})$, suponhamos que posto de $A$ na origem seja s. Quando $\mathbb{K}=\mathbb{C}$ então $A$ é $\mathcal{G}$-equivalente a um germe da forma $I_{s} \oplus B$, onde $B:\left(\mathbb{C}^{r}, 0\right) \rightarrow \operatorname{Sim}_{(n-s)}(\mathbb{C})$ tem posto nulo na origem e $I_{s}$ é a matriz identidade $s \times s$. Sobre $\mathbb{R}$ trocamos $I_{s}$ por uma matriz com \pm 1 's na diagonal. A $\mathcal{G}$-codimensão de $A$ é igual à de $B$, seus desdobramentos são naturalmente isomórficos e seus discriminantes coincidem. A simplicidade de um é equivalente à simplicidade do outro. Dizemos que A é uma suspensão de $B$.

O resultado acima nos mostra que podemos apenas considerar germes que se anulam na origem. Sendo assim, passaremos a utilizar o método tradicional de classificação apresentado na Seção 1.1, para o qual os principais resultados são: Transversal Completa, Lema de Mather e critérios de determinação finita. 
A Transversal Completa pode produzir uma família de jatos que são todos distintos sob a $\mathcal{G}$-equivalência. Neste caso, dizemos que esta família possui módulo e que o germe é não simples. O próximo resultado nos fornece um critério para detectarmos a existência de módulo e o utilizaremos para descartar os casos de germes não simples.

Proposição 2.5.4. Sejam $V$ um $\mathbb{K}$-espaço afim e $G$ um grupo algébrico de Lie agindo em $V$. Se $W$ é uma subvariedade suave de $V$ com a propriedade de que o conjunto $\left\{w \in W ; \mathrm{T}_{w} W \subset\right.$ $\left.\mathrm{T}_{w} G . w\right\}$ é uma subvariedade própria de $W$ então dado qualquer $w \in W$, para toda vizinhança $U$ de $w$ temos que ela encontra um número infinito de G-órbitas.

Observação 2.5.5. Em particular, se $\operatorname{dim} W>\operatorname{dim} G$ então temos módulo. De fato, lembrando que $\operatorname{dim} T_{w} G . w \leqslant \operatorname{dim} G$, temos que

$$
\operatorname{dim} T_{w} G w \leqslant \operatorname{dim} G<\operatorname{dim} W
$$

logo o conjunto $\left\{w \in W ; \mathrm{T}_{w} W \subset \mathrm{T}_{w} G . w\right\}$ será vazio, segue da Proposição 2.5.4 que temos módulo. Em particular, tomando $W=V$, temos que se $\operatorname{dim} V>\operatorname{dim} G$, então temos módulo.

Observação 2.5.6. A Proposição 2.2.9 fornece uma ferramenta importante para a verificação de módulo. Se a singularidade do discriminante de uma matriz possui um módulo que pode ser realizado através da variação dos coeficientes da matriz então, por esta proposição, a matriz é também não simples.

Como estamos interessados em classificar germes suaves $A: \mathbb{K}^{r} \rightarrow \operatorname{Sim}_{n}(\mathbb{K})$, que sejam $\mathcal{G}$-simples, vamos primeiramente detectar os casos em que eles não podem aparecer. Segue do Lema 1.1.16, que se um elemento $A \in \mathcal{S}$ possui 1-jato $\alpha \in \operatorname{Hom}\left(\mathbb{K}^{r}, \operatorname{Sim}_{n}(\mathbb{K})\right)$ no qual qualquer vizinhança de $\alpha$ encontra infinitas órbitas em $J^{1} \mathcal{G}$ então $A$ é não simples. Usando esta idéia obtemos os seguintes resultados:

Proposição 2.5.7. Não há germes simples se:

- $r=n=3$;

- $n \geqslant 4$ e $2 \leqslant r \leqslant\left(n^{2}+n-4\right) / 2$.

Demonstração. A ação do grupo $J^{1} \mathcal{G}$ no espaço dos 1 -jatos de $\mathcal{S}$, se reduz à ação de $G L_{r}(\mathbb{K})$ e $G L_{n}(\mathbb{K})$ em $\operatorname{Hom}\left(\mathbb{K}^{r}, S i m_{n}(\mathbb{K})\right)$. A dimensão do grupo $G L_{r}(\mathbb{K}) \times G L_{n}(\mathbb{K})$ é $r^{2}+n^{2}$ e do espaço $\frac{r n(n+1)}{2}$. Pela Observação 2.3.3, a dimensão de cada órbita nesta ação é no máximo $r^{2}+n^{2}-1$. Segue da Observação 2.5.5 que se $\frac{r n(n+1)}{2} \geqslant r^{2}+n^{2}$ então não há germes simples. Verifiquemos para quais valores de $r, n$ é satisfeita a inequação:

$$
r n^{2}+r n-2 r^{2}-2 n^{2} \geqslant 0
$$


Para $n=1$ ou $n=2$, a inequação (2.1) nunca vale. De fato, para $n=1$ e $n=2$, temos respectivamente:

$$
\begin{array}{r}
r+r-2 r^{2}-2=2\left(-r^{2}+r-1\right) \\
4 r+2 r-2 r^{2}-8=2\left(-r^{2}+3 r-4\right)
\end{array}
$$

Usando Bhaskara temos que o discriminante das expressões $\left(-r^{2}+r-1\right)$ e $\left(-r^{2}+3 r-4\right)$, são -3 e -7 , respectivamente. Deste modo, ambas equações não possuem raiz real e assim $\left(-r^{2}+r-1\right)<0$ e $\left(-r^{2}+3 r-4\right)<0, \forall r \in \mathbb{N}$.

Para $n=3$, temos

$$
9 r+3 r-2 r^{2}-18=-2\left(r^{2}-6 r+9\right)=-2(r-3)^{2}
$$

Portanto a inequação (2.1) é satisfeita para $n=3$ somente se $\mathrm{r}=3$.

Afirmação: Se $n \geqslant 4$ e $r \geqslant 2$, a inequação (2.1) vale se, e somente se, $r \leqslant \frac{n(n+1)}{2}-2$.

De fato, sejam $n \geqslant 4$ e $r \geqslant 2$, fixos. Suponha que a inequação (2.1) é satisfeita para esses valores de $r$ e $n$. Assim, são equivalentes:

$$
\begin{array}{r}
r n^{2}+r n-2 r^{2}-2 n^{2} \geqslant 0 \\
r\left(n^{2}+n-2 r\right) \geqslant 2 n^{2} \\
r\left(\frac{n^{2}+n-2 r}{2}\right) \geqslant n^{2} .
\end{array}
$$

Como $n \geqslant 4$ e $r \geqslant 2$, se (2.1) é satisfeita, temos

$$
\frac{n^{2}+n-2 r}{2}>0
$$

e ainda, $r, n \in \mathbb{N} \Rightarrow \frac{n(n+1)-2 r}{2} \in \mathbb{N}$. Podemos afirmar que $\frac{n^{2}+n-2 r}{2}>1$. Com efeito, se supormos que $\frac{n^{2}+n-2 r}{2}=1$, segue que

$$
r(\underbrace{\frac{n^{2}+n-2 r}{2}}_{=1}) \geqslant n^{2} \Rightarrow r \geqslant n^{2} .
$$

Usando que $n \geqslant 4 \Rightarrow n<n^{2} \leqslant r$, $\operatorname{logo} n^{2}+n-2 r<0$ o que contradiz (2.2). Portanto $\frac{n^{2}+n-2 r}{2}>1$, ou seja, $\frac{n^{2}+n-2 r}{2} \geqslant 2 \Rightarrow \frac{n^{2}+n}{2}-2 \geqslant r$.

Por outro lado, suponha $n \geqslant 4$ e $2 \leqslant r \leqslant \frac{n^{2}+n}{2}-2$, queremos mostrar que para quaisquer $r$ e $n$ nessas condições (2.1) se verifica.

Para tanto, seja $n \geqslant 4$ fixo e considere: 


$$
\begin{aligned}
g(r) & =r n^{2}+r n-2 r^{2}-2 n^{2} \\
& =-2 r^{2}+r\left(n^{2}+n\right)-2 n^{2} .
\end{aligned}
$$

Calculando as raízes de $g$, obtemos:

$$
r_{1}=\frac{\left(n^{2}+n\right)-\sqrt{\left(n^{2}+n\right)^{2}-16 n^{2}}}{4} ; r_{2}=\frac{\left(n^{2}+n\right)+\sqrt{\left(n^{2}+n\right)^{2}-16 n^{2}}}{4}
$$

Logo, para todo $r$ tal que $r_{1} \leqslant r \leqslant r_{2}$, temos $g(r) \geqslant 0$, ou seja, (2.1) é satisfeita. Sendo assim, se mostrarmos que $r_{1} \leqslant 2$ e $\frac{n^{2}+n}{2}-2 \leqslant r_{2}$, o resultado segue.

Mostremos que $r_{1} \leqslant 2$.

Como $4 \leqslant n$, temos:

$$
\begin{aligned}
64 & \leqslant 16 n \\
-16 n+64 & \leqslant 0 \\
-16 n^{2}-16 n+64 & \leqslant-16 n^{2} \\
\left(n^{2}+n-8\right)^{2} & \leqslant\left(n^{2}+n\right)^{2}-16 n^{2} \\
n^{2}+n-8 & \leqslant \sqrt{\left(n^{2}+n\right)^{2}-16 n^{2}} \\
\frac{n^{2}+n-\sqrt{\left(n^{2}+n\right)^{2}-16 n^{2}}}{n^{2}+n-\sqrt{\left(n^{2}+n\right)^{2}-16 n^{2}}} & \leqslant 2 \\
4 & \leqslant 2 .
\end{aligned}
$$

Mostremos que $\frac{n^{2}+n}{2}-2 \leqslant r_{2}$.

Como $4 \leqslant n$, temos:

$$
\begin{aligned}
64 & \leqslant 16 n \\
-16 n+64 & \leqslant 0 \\
\left(n^{2}+n\right)^{2}-2(8)\left(n^{2}+n\right)+64 & \leqslant\left(n^{2}+n\right)^{2}-16 n^{2} \\
\left(n^{2}+n-8\right)^{2} & \leqslant\left(n^{2}+n\right)^{2}-16 n^{2} ;(\text { ambos os lados são positivos) } \\
n^{2}+n-8 & \leqslant \sqrt{\left(n^{2}+n\right)^{2}-16 n^{2}} \\
2 n^{2}+2 n-8 & \leqslant n^{2}+2 n+\sqrt{\left(n^{2}+n\right)^{2}-16 n^{2}} \\
\frac{n^{2}+n}{2}-2 & \leqslant \frac{n^{2}+2 n+\sqrt{\left(n^{2}+n\right)^{2}-16 n^{2}}}{4} \\
\frac{n^{2}+n}{2}-2 & \leqslant r_{2} .
\end{aligned}
$$

Seja $A:\left(\mathbb{K}^{r}, 0\right) \rightarrow \operatorname{Sim}_{n}(\mathbb{K})$ e considere seu 1-jato $\alpha \in H o m\left(\mathbb{K}^{r}, \operatorname{Sim}_{n}(\mathbb{K})\right)$ então temos que posto $(\alpha)=$ posto $(d A(0))$ e coposto $(\alpha)=$ coposto $(d A(0))$. Usando este conceito e a proposição anterior, podemos melhorar a descrição dos casos que nos interessam.

Proposição 2.5.8. Temos que $\alpha$ não é $\mathcal{G}$-simples a menos que um dos seguintes casos ocorra:

- $n=1,2$ ou $r=1$; 
- $r=2$ e $n=3$;

- $\operatorname{para} n \geqslant 3$, coposto $(\alpha)=0$ ou 1 ;

- se $n=3$ então posto $(\alpha)=4$.

Demonstração. Seja $\alpha \in \operatorname{Hom}\left(\mathbb{K}^{r}, \operatorname{Sim}_{n}(\mathbb{K})\right)$ e suponha que coposto $(\alpha)=s$. Pelo Lema 2.1.12 o núcleo da aplicação dual $\alpha^{*}: \operatorname{Sim}_{n}^{*} \rightarrow\left(\mathbb{K}^{r}\right)^{*}$ tem dimensão $s$. Este núcleo é um subespaço $s$-dimensional de um espaço de dimensão $N=\frac{n(n+1)}{2}$, ou seja, é um elemento de uma Grasmaniana de dimensão $(N-s) s$.

Temos uma ação do projetivo do grupo linear $P G L_{n}$ nesse espaço. Pela Observação 2.5.5, temos módulo quando:

$$
s\left[\frac{n(n+1)}{2}-s\right] \geqslant n^{2}, \text { ou seja }, s n^{2}+n s-2 s^{2}-2 n^{2} \geqslant 0
$$

Mas note que pelas contas da proposição anterior essa inequação só é satisfeita para:

- $n \geqslant 4$ e $2 \leqslant s \leqslant \frac{n^{2}+n-4}{2}$ ou

- $n=s=3$.

Agora, se $n \geqslant 4$, para que não haja módulo devemos ter $\frac{n^{2}+n-4}{2}<s$ ou $s<2$. Mas, quando $\frac{n^{2}+n-4}{2}<s$, temos $\underbrace{\frac{n^{2}+n}{2}-s}_{\text {posto }(\alpha)}<2$. Logo, posto $(\alpha)$ deve ser 0 ou 1. Assim para $n \geqslant 4$, temos posto $(\alpha) \leqslant 1$ ou coposto $(\alpha) \leqslant 1$.

Mas quando $r \geqslant 2$ os 1 -jatos de posto 0 ou 1 têm órbita adjacente de posto 2 , que tem módulo. Portanto, se $n \geqslant 4$, poderemos encontrar germes simples somente quando $r=1 \mathrm{ou}$ $\operatorname{coposto}(\alpha)=0$ ou coposto $(\alpha)=1$.

Quando $n=3$, pela proposição anterior para encontrarmos germes simples devemos ter $r \neq 3$ e pelo que foi dito acima também devemos ter $s \neq 3$. Assim, se $r>3$ então para que não haja módulo devemos ter $s>3$ ou $s<3$. Se $s=6-\operatorname{posto}(\alpha)>3 \Rightarrow 3>$ posto $(\alpha)$, logo qualquer elemento nessas condições possui órbita adjacente de posto 3, que tem módulo. Portanto, para $n=3$ podemos encontrar germes simples se $r=1, r=2$ ou posto( $\alpha)$ igual a $4 ; 5$ ou 6 . Não há condição quando $n=1,2$.

Sendo assim, para encontrarmos germes simples devemos ter

- $n=1,2$ ou $r=1$

- $(n=3$ e $r=2)$ ou $(n=3$ e posto $(\alpha)=4,5,6)$

- $\operatorname{para} n \geqslant 4$ posto $(\alpha)=0 ; 1$

Claramente essas condições são equivalentes àquelas enunciadas na proposição. 


\subsection{Classificação dos 1-jatos provenientes dos pencils de quadráticas}

Vimos na Proposição 2.5.8, quais valores de $r$ e $n$ podem nos fornecer germes simples, entre eles temos $n=2$ e $n=3$. A classificação dos 1 -jatos $\alpha: \mathbb{K}^{r} \rightarrow \operatorname{Sim}_{n}(\mathbb{K})$ para estes casos são provenientes dos pencils de quadráticas em 2 e 3 variáveis, respectivamente. Para obter estas formas normais vamos usar resultados que relacionam formas quadráticas com matrizes simétricas e resultados de dualidade (ver Seção 2.1).

Analisemos quando $n=2$. Dado o 1 -jato $\alpha \in J^{1} \mathcal{S}$ temos que posto $(\alpha) \leqslant 3$. O caso posto $(\alpha)=3$ será tratado na Proposição 3.1.2. Assim, resta analisarmos quando posto $(\alpha) \leqslant 2$. Fazendo mudanças na fonte de $\alpha$ podemos escrevê-lo da forma:

$$
\alpha=\left(\begin{array}{ll}
a_{1} x+b_{1} y & a_{2} x+b_{2} y \\
a_{2} x+b_{2} y & a_{3} x+b_{3} y
\end{array}\right)=x\left(\begin{array}{ll}
a_{1} & a_{2} \\
a_{2} & a_{3}
\end{array}\right)+y\left(\begin{array}{ll}
b_{1} & b_{2} \\
b_{2} & b_{3}
\end{array}\right)=x B_{1}+y B_{2} .
$$

A ação de $J^{1} \mathcal{G}$ nos 1-jatos se reduz à ação de $G L_{2}(\mathbb{C}) \times G L_{2}(\mathbb{C})$ em $\operatorname{Hom}\left(\mathbb{K}^{2}, \operatorname{Sim}_{2}(\mathbb{K})\right)$. A ação na meta desses elementos nada mais é do que a ação de $G L_{2}(\mathbb{K})$ em $\operatorname{Sim}_{2}(\mathbb{K})$ e a ação na fonte é uma mudança na base do espaço gerado por $B_{1}$ e $B_{2}$. Associando as matrizes $B_{1}, B_{2}$ com as suas formas quadráticas correspondentes e usando a Proposição 2.1.7, segue que classificar os 1-jatos pela ação de $G L_{2}(\mathbb{K}) \times G L_{2}(\mathbb{K})$, é equivalente a classificar pencil de quadrática pela ação de $G L_{2}(\mathbb{K}) \times G L_{2}(\mathbb{K})$. A partir de agora, trabalhemos sobre $\mathbb{C}$.

Proposição 2.6.1. Os pencils de quadráticas sobre $\mathbb{C}$ possuem as seguintes formas normais:

$$
\left(x^{2}+y^{2}, x y\right),\left(x^{2}, x y\right),\left(x^{2}+y^{2}, 0\right),\left(x^{2}, 0\right),(0,0)
$$

Demonstração. Ver [13].

Usando o isomorfismo dado na Proposição 2.1.4, façamos corresponder para cada coordenada do pencil a sua matriz correspondente $B_{i}$. Assim, obtemos o 1-jato $x B_{1}+y B_{2} \in$ $\operatorname{Hom}\left(\mathbb{C}^{r}, \operatorname{Sim}_{2}(\mathbb{C})\right)$. Por exemplo, para o pencil $\left(x^{2}+y^{2}, x y\right)$ temos

$$
x^{2}+y^{2}=\left(\begin{array}{ll}
x & y
\end{array}\right)\left(\begin{array}{ll}
1 & 0 \\
0 & 1
\end{array}\right)\left(\begin{array}{l}
x \\
y
\end{array}\right) \text { e } x y=\left(\begin{array}{ll}
x & y
\end{array}\right)\left(\begin{array}{ll}
0 & 1 \\
1 & 0
\end{array}\right)\left(\begin{array}{l}
x \\
y
\end{array}\right) .
$$

Portanto, o 1-jato correspondente ao pencil $\left(x^{2}+y^{2}, x y\right)$ é dado por

$$
x\left(\begin{array}{ll}
1 & 0 \\
0 & 1
\end{array}\right)+y\left(\begin{array}{ll}
0 & 1 \\
1 & 0
\end{array}\right)=\left(\begin{array}{ll}
x & y \\
y & x
\end{array}\right) .
$$

Analogamente para os outros pencils obtemos os seguintes 1-jatos: 


$$
\begin{aligned}
\left(x^{2}+y^{2}, x y\right) & \rightarrow x\left(\begin{array}{ll}
1 & 0 \\
0 & 1
\end{array}\right)+y\left(\begin{array}{ll}
0 & 1 \\
1 & 0
\end{array}\right)=\left(\begin{array}{ll}
x & y \\
y & x
\end{array}\right), \\
\left(x^{2}, x y\right) & \rightarrow x\left(\begin{array}{ll}
1 & 0 \\
0 & 0
\end{array}\right)+y\left(\begin{array}{ll}
0 & 1 \\
1 & 0
\end{array}\right)=\left(\begin{array}{ll}
x & y \\
y & 0
\end{array}\right), \\
\left(x^{2}+y^{2}, 0\right) & \rightarrow x\left(\begin{array}{ll}
1 & 0 \\
0 & 1
\end{array}\right)+y\left(\begin{array}{ll}
0 & 0 \\
0 & 0
\end{array}\right)=\left(\begin{array}{ll}
x & 0 \\
0 & x
\end{array}\right), \\
\left(x^{2}, 0\right) & \rightarrow x\left(\begin{array}{ll}
1 & 0 \\
0 & 0
\end{array}\right)+y\left(\begin{array}{ll}
0 & 0 \\
0 & 0
\end{array}\right)=\left(\begin{array}{ll}
x & 0 \\
0 & 0
\end{array}\right) .
\end{aligned}
$$

Portanto, temos:

Proposição 2.6.2. Sobre $\mathbb{C}$, quando $n=2$, as possíveis órbitas dos 1-jatos são:

$$
\left(\begin{array}{ll}
x & y \\
y & x
\end{array}\right),\left(\begin{array}{ll}
x & y \\
y & 0
\end{array}\right),\left(\begin{array}{ll}
x & 0 \\
0 & x
\end{array}\right),\left(\begin{array}{ll}
x & 0 \\
0 & 0
\end{array}\right) e\left(\begin{array}{ll}
0 & 0 \\
0 & 0
\end{array}\right) .
$$

Por outro lado, quando $n=3$ temos $\operatorname{posto}(\alpha) \leqslant 6$. Pela Proposição 2.5.8 podemos obter germes simples quando $r=1, r=2$ ou posto $(\alpha)=4,5,6$. O caso $r=1$ será tratado na Proposição 3.1.4 e os casos em que posto $(\alpha)=5,6$ serão tratados na Proposição 3.1.2. O caso $r=2$ e posto $(\alpha)=1$ será tratado na Proposição 3.2.1. Logo, resta considerarmos os casos em que posto $(\alpha)=4$ e o caso em que $r=2$, mas posto $(\alpha)=2$. Pelo Lema 2.1.12 e pela Proposição 2.1.14, classificar subespaços de dimensão 2 em $\operatorname{Sim}_{3}(\mathbb{C})$ é equivalente a classificar subespaços de dimensão $4 \mathrm{em} \operatorname{Sim}_{3}(\mathbb{C})$. Vamos usar este fato para simplificar esta classificação.

Pelos mesmos argumentos feitos no caso em que $n$ era igual a 2, segue que classificar os 1-jatos pela ação de $J^{1} \mathcal{G}$ é equivalente a classificar pencils de quadráticas em três variáveis, ou seja, cônicas. Para esta classificação é usado o conceito de Símbolo Segre que aparece entre chaves na Proposição 2.6.3. Não utilizaremos estes símbolos, porém apresentamos um resumo desta teoria no Apêndice B. No caso de cônicas temos 12 formas normais, ver ([27], p. 477). Porém quatro dessas formas normais fornecem pencils em que uma das cônicas se anula identicamente. Pelo que foi dito acima, estamos apenas interessados em pencils que produzam espaços de dimensão 2. Logo, temos:

Proposição 2.6.3. As formas normais dos pencils de cônicas, junto com os seus Símbolos Segre, são os seguintes:

$$
\begin{gathered}
\left(x y, x^{2}+y^{2}+z^{2}\right),\{1,1,1\} ;\left(x y, y^{2}+z^{2}\right),\{2,1\} ;\left(2 x z+y^{2}, x y\right),\{3\} \\
\left(x y, z^{2}\right),\{(1,1), 1\} ;\left(x^{2}+2 y z, y^{2}\right),\{(2,1)\} ;\left(x^{2}, y^{2}\right),\{1,1 ; 1\} \\
(x y, x z),\{; 2\} ;\left(x^{2}, x y\right),\{2 ; 1\} .
\end{gathered}
$$


Através destes pencils passemos a obter as formas normais dos 1-jatos de matrizes quando $n=3$ para os casos $r=2$ e posto $(\alpha)=4$.

Para o caso $r=2, n=3$ e posto $(\alpha)=2$, seguimos o mesmo processo de $r=n=2$, onde a cada pencil associamos duas matrizes $B_{1}, B_{2}$ e o 1-jato será $x B_{1}+y B_{2}$. Para que fique claro, tomando por exemplo o pencil $\left(x y, y^{2}+z^{2}\right)$ temos:

$$
x y=\left(\begin{array}{lll}
x & y & z
\end{array}\right)\left(\begin{array}{lll}
0 & 1 & 0 \\
1 & 0 & 0 \\
0 & 0 & 0
\end{array}\right)\left(\begin{array}{l}
x \\
y \\
z
\end{array}\right) \text { e } y^{2}+z^{2}=\left(\begin{array}{lll}
x & y & z
\end{array}\right)\left(\begin{array}{lll}
0 & 0 & 0 \\
0 & 1 & 0 \\
0 & 0 & 1
\end{array}\right)\left(\begin{array}{l}
x \\
y \\
z
\end{array}\right) .
$$

Logo, o 1-jato correspondente a esse pencil é dado por:

$$
x\left(\begin{array}{lll}
0 & 1 & 0 \\
1 & 0 & 0 \\
0 & 0 & 0
\end{array}\right)+y\left(\begin{array}{lll}
0 & 0 & 0 \\
0 & 1 & 0 \\
0 & 0 & 1
\end{array}\right)=\left(\begin{array}{lll}
0 & x & 0 \\
x & y & 0 \\
0 & 0 & y
\end{array}\right) .
$$

Fazendo o mesmo para cada pencil obtemos:

Proposição 2.6.4. Sobre $\mathbb{C}$, quando $r=2$ e $n=3$ as possíveis órbitas dos 1 -jatos de posto 2 são:

$$
\begin{aligned}
\left(\begin{array}{lll}
y & x & 0 \\
x & y & 0 \\
0 & 0 & y
\end{array}\right), & \left(\begin{array}{lll}
0 & x & 0 \\
x & y & 0 \\
0 & 0 & y
\end{array}\right),\left(\begin{array}{lll}
0 & y & x \\
y & x & 0 \\
x & 0 & 0
\end{array}\right),\left(\begin{array}{lll}
0 & x & 0 \\
x & 0 & 0 \\
0 & 0 & y
\end{array}\right),\left(\begin{array}{lll}
x & 0 & 0 \\
0 & y & x \\
0 & x & 0
\end{array}\right), \\
& \left(\begin{array}{lll}
x & 0 & 0 \\
0 & y & 0 \\
0 & 0 & 0
\end{array}\right),\left(\begin{array}{lll}
0 & x & y \\
x & 0 & 0 \\
y & 0 & 0
\end{array}\right),\left(\begin{array}{lll}
x & y & 0 \\
y & 0 & 0 \\
0 & 0 & 0
\end{array}\right) .
\end{aligned}
$$

Para o caso $n=3$ e posto $(\alpha)=4$, temos que a Proposição 2.6.3 nos fornece germes lineares de posto 2, que são provenientes dos pencils. Lembremos que pela dualidade fornecida no Lema 2.1.12, classificar subespaços de dimensão 2 nas simétricas é equivalente a classificar subespaços de dimensão 4. Assim, pela dualidade, cada família dos 1-jatos de posto 4 é gerada pelo espaço ortogonal ao espaço dado pelos geradores do pencil. Ou seja, se um pencil é gerado por $B_{1}, B_{2}$, então a família dos 1 -jatos $A$, consiste dos germes tais que $\operatorname{tr}\left(B_{i} A\right)=0$, para $\mathrm{i}=1,2$.

Por exemplo, tomando o pencil $\left(x y, x^{2}+y^{2}+z^{2}\right)$ que é gerado por $B_{1}=E_{12}$ e $B_{2}=$ $E_{11}+E_{22}+E_{33}$, temos que o germe $A=\left(\begin{array}{ccc}a_{11} & a_{12} & a_{13} \\ a_{12} & a_{22} & a_{23} \\ a_{13} & a_{23} & a_{33}\end{array}\right)$ deve satisfazer:

$$
\left\{\begin{aligned}
a_{11}+a_{22}+a_{33} & =0 \\
a_{12} & =0
\end{aligned}\right. \text {. }
$$


Assim, esse pencil nos fornece o elemento $\left(\begin{array}{ccc}x & 0 & z \\ 0 & y & w \\ z & w & -x-y\end{array}\right)$. Logo, temos:

Proposição 2.6.5. Sobre $\mathbb{C}$, quando $n=3$ as possiveis órbitas dos 1 -jatos de posto 4 são:

$$
\begin{gathered}
\left(x y, x^{2}+y^{2}+z^{2}\right),\left(\begin{array}{ccc}
x & 0 & z \\
0 & y & w \\
z & w & -x-y
\end{array}\right) ;\left(x y, y^{2}+z^{2}\right),\left(\begin{array}{ccc}
x & 0 & z \\
0 & y & w \\
z & w & -y
\end{array}\right) ; \\
\left(2 x z+y^{2}, x y\right),\left(\begin{array}{ccc}
x & 0 & y \\
0 & -y & z \\
y & z & w
\end{array}\right) ;\left(x y, z^{2}\right),\left(\begin{array}{ccc}
x & 0 & z \\
0 & y & w \\
z & w & 0
\end{array}\right) ;\left(x^{2}+2 y z, y^{2}\right),\left(\begin{array}{ccc}
x & z & w \\
z & 0 & -x \\
w & -x & y
\end{array}\right) ; \\
\left(x^{2}, y^{2}\right),\left(\begin{array}{ccc}
0 & y & z \\
y & 0 & w \\
z & w & x
\end{array}\right) ;(x y, x z),\left(\begin{array}{ccc}
x & 0 & 0 \\
0 & y & w \\
0 & w & z
\end{array}\right) ;\left(x^{2}, x y\right),\left(\begin{array}{ccc}
0 & 0 & z \\
0 & x & w \\
z & w & y
\end{array}\right) .
\end{gathered}
$$




\section{Capítulo 3}

\section{Classificação}

O objetivo deste capítulo é classificar germes simples $A: \mathbb{C}^{r} \rightarrow \operatorname{Sim}_{n}(\mathbb{C})$ e exibir formas normais para eles. Essa classificação é feita utilizando resultados como a Transversal Completa, Lema de Mather e critérios de determinação finita. O fato de serem germes simples pode (como de costume) ser deduzido utilizando seus desdobramentos versais.

No capítulo anterior obtivemos os possíveis casos em que o 1-jato de $A$ pode ser simples. Vamos ver que alguns deles geram ainda germes não simples, portanto não serão considerados em nossa classificação. A classificação dos germes simples foi dividida em 5 casos, sendo que cada caso se encontra em uma seção deste capítulo.

Vimos na Proposição 2.5.8 que em alguns casos ser simples está relacionado ao posto ou coposto do 1-jato de $A$, comecemos com alguns destes casos.

\subsection{Os casos coposto 0 , coposto $1, r=1$}

Seja $A:\left(\mathbb{C}^{r}, 0\right) \rightarrow \operatorname{Sim}_{n}(\mathbb{C}),(\operatorname{com} A(0)=0)$ e seu 1-jato $\alpha \in \operatorname{Hom}\left(\mathbb{C}^{r}, \operatorname{Sim}_{n}(\mathbb{C})\right)$. Lembremos que posto $(\alpha)=$ posto $(d(A(0))$ e coposto $(\alpha)=$ coposto $(d(A(0))$. O próximo teorema resume as Proposições 3.1.2 e 3.1.3, que classificam os germes cujo 1-jato tem coposto 0 ou 1.

Teorema 3.1.1. (1) Quando coposto $(\alpha)=0$ temos uma forma normal $A: \mathbb{C}^{N} \times \mathbb{C}^{s} \rightarrow$

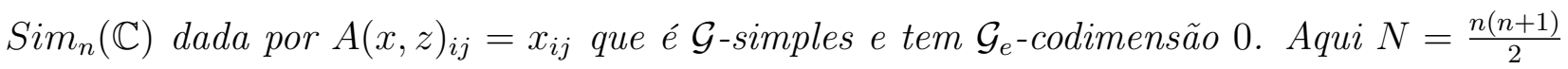
$e$ as variáveis z são redundantes.

(2) Quando coposto $(\alpha)=1$ temos dois casos:

(i) Uma forma normal $A: \mathbb{C}^{N-1} \times \mathbb{C}^{s} \rightarrow \operatorname{Sim}_{n}(\mathbb{C})$ dada por $A(x, z)_{i j}=x_{i j}$ para $(i j) \neq(11)$ e $A(x, z)_{11}=\sum_{i=2}^{n} x_{i i}+f(z)$, onde $f:\left(\mathbb{C}^{s}, 0\right) \rightarrow \mathbb{C}$ é um germe $\mathcal{R}$-simples $([2])$.

(ii) Uma forma normal $A: \mathbb{C}^{N-1} \times \mathbb{C}^{s} \rightarrow \operatorname{Sim}_{n}(\mathbb{C})$ é dada por $A(x, z)_{i j}=x_{i j}$ para $(i j) \neq(11)$ e $A(x, z)_{11}=\sum_{i=2}^{n-1} x_{i i}+f\left(x_{n n}, z\right)$, onde $f:\left(\mathbb{C} \times \mathbb{C}^{s}, 0\right) \rightarrow \mathbb{C}$ é um germe 
simples de uma função de variedade com bordo ([3]).

Proposição 3.1.2. (1) Se coposto $(\alpha)=0$ então o 1-jato é uma submersão. Qualquer germe $A \in S$ com tal 1-jato é 1-G-determinado e de fato G-estável no sentido natural. Uma forma normal é obtida colocando uma diferente função coordenada em cada posição.

(2) Se coposto $(\alpha)=1$, então $r \geq \frac{n(n+1)}{2}-1$ e denominamos as primeiras $\frac{n(n+1)}{2}-1$ das $x$-variáveis por $x_{i j}$ com $1 \leq i, j \leq n,(i, j) \neq(1,1)$. Então $\alpha$ é equivalente ao 1-jato da forma:

$$
\left(\sum_{i=2}^{t} x_{i i}\right) E_{11}+\sum_{(i, j) \neq(1,1)} x_{i j} E_{i j},
$$

onde $E_{i j}$ é a matriz com 1 na $(i, j)$-ésima e $(j, i)$-ésima posições e zeros nas outras e $1 \leq t \leq n$ (o caso $t=1$ é interpretado com a primeira soma ausente).

Demonstração. (1) Basta usarmos o Teorema das Submersões.

(2) Temos que $\alpha \in \operatorname{Hom}\left(\mathbb{C}^{r}, \operatorname{Sim}_{n}(\mathbb{C})\right)$ e por hipótese coposto $(\alpha)=1$, ou seja, posto $(\alpha)=$ $\frac{n(n+1)}{2}-1$ e consequentemente $r \geq \frac{n(n+1)}{2}-1$. Pela dualidade fornecida no Lema $2.1 .12 \mathrm{e}$ na Proposição 2.1.14, basta classificarmos subespaços 1-dimensionais de $\operatorname{Sim}_{n}(\mathbb{C})$ pela nossa equivalência. Sabemos que as formas normais por essa ação são dadas por $I_{t}^{*}$, com $1 \leqslant t \leqslant n$. Logo, para obtermos as formas normais dos 1-jatos com coposto 1, devemos exibir para cada $t$, um 1-jato em que sua imagem seja $\left(I_{t}^{*}\right)^{\perp}=\left\{X \in \operatorname{Sim}_{n}(\mathbb{C}) ; \operatorname{traço}\left(X I_{t}^{*}\right)=0\right\}$. Os elementos da forma $\sum_{i=2}^{t}-x_{i i} E_{11}+\sum_{(i, j) \neq(1,1)} x_{i j} E_{i j}$, satisfazem esta condição e claramente os 1-jatos descritos na proposição são $J^{1} \mathcal{G}$-equivalentes a estes.

Proposição 3.1.3. A classificação de germes com 1-jato dados na Proposição 3.1.2, item (2), reduz à classificação de germes de funções $\left(\mathbb{C}^{s} \times \mathbb{C}^{M},(0,0)\right) \mapsto \mathbb{C}$, onde $M=\frac{m(m+1)}{2}$, $m=n-t$, e $\mathbb{C}^{M}$ é considerado o espaço das matrizes simétricas $m \times m$ com um discriminante $\Delta$. O grupo relevante de equivalência é o produto semi-direto do grupo de difeomorfismos preservando $\mathbb{C}^{s} \times \Delta$ e o grupo usual $\mathcal{C}$ para germes de funções.

Não há singularidades simples se $m \geq 2$, ou seja, se $t \leq n-2$. Assim precisamos somente considerar os casos $t=n$ e $t=n-1$. Estes reduzem respectivamente à classificação de funções, funções em uma variedade com bordo, com o grupo $\mathcal{C}$ presente.

Demonstração. Seja um 1-jato $A$ como na Proposição 3.1.2, item (2). Assim, $A$ é da forma:

$$
A=\left(\begin{array}{ccccc}
\sum_{i=2}^{t} x_{i i} & x_{12} & \ldots & \ldots & x_{1 n} \\
x_{12} & x_{22} & \ldots & \ldots & \ldots \\
\vdots & \ldots & \ldots & \ldots & \ldots \\
\vdots & \ldots & \ldots & \ldots & \ldots \\
x_{1 n} & \ldots & \ldots & \ldots & x_{n n}
\end{array}\right)
$$


Calculemos uma 2-transversal completa para $A$. Usando os elementos $A_{x_{i j}}$, com $i \neq$ $j$, obtemos todos os elementos de $H^{2} \mathcal{S}$ em todas as entradas fora da diagonal e com os elementos $A_{x_{i i}}$, para $t \leqslant i \leqslant n$, obtemos todos os elementos de $H^{2} \mathcal{S}$ nas $(t+1)$-ésima até a $n$-ésima posição da diagonal. Para $2 \leqslant i \leqslant t$, usando os elementos $A_{i j}$ e $A_{x_{i i}}$ obtemos todos os elementos do ideal $\mathcal{M}_{r}\left\langle x_{i j}, 1 \leq j \leq n\right.$ e $\left.2 \leqslant i \leqslant t\right\rangle$ na posição $(1,1)$ e com os elementos $A_{1 j}$ obtemos todos os elementos do ideal $\mathcal{M}_{r}\left\langle x_{1 j}, 2 \leq j \leq n\right\rangle$ na mesma posição. Consequentemente com $A_{x_{i i}}$ temos estes mesmos ideais na $i$-ésima posição da diagonal. Temos também todos os múltiplos de $p\left(E_{11}+E_{i i}\right)$, com $p \in H^{2} \mathcal{S}$.

Suponhamos que há s variáveis $y_{1}, \ldots, y_{s}$ não aparecendo neste 1 -jato, $\log o r=\frac{n(n+1)}{2}+s-1$. Pelo que foi feito acima, segue que uma 2-transversal completa é obtida adicionando um termo geral $h(X, z)$ na entrada $(1,1)$ da matriz $A$, onde $X$ denota as variáveis $x_{i j}$ com $t+1 \leq i \leq$ $j \leq n$ e $z=\left(y_{1}, \ldots, y_{s}\right)$.

Podemos fazer todas as mudanças de coordenadas nas variáveis $z$. Porém, se nós queremos preservar a forma da transversal completa, as mudanças de coordenadas nas variáveis $X$ devem ser recuperadas pela ação do grupo $\mathcal{H}$.

Isto significa, que se $\mathcal{H}_{t}$ é o subgrupo de $\mathcal{H}$ correspondente às matrizes da forma $I \oplus B$, onde $I$ é a matriz identidade $t \times t$ e $B$ é uma matriz invertível $m \times m$, então podemos fazer mudanças de coordenadas nas variáveis $X$ pela ação $X \mapsto B^{t} X B$. Isto resulta, que se duas funções $h_{1}(X, z)$ e $h_{2}(X, z)$ são equivalentes por esta ação então os correspondentes germes são $\mathcal{G}$-equivalentes. Por outro lado, podemos checar que os espaços normais pela $\mathcal{G}$-ação para germes de aplicação e o do $\mathcal{K}_{\Delta}$-ação das correspondentes germes de funções são isomorfos. Em outras palavras, temos identificado um subgrupo de $\mathcal{G}$ que em todo o espaço dos jatos a dimensão das órbitas de $J^{k} \mathcal{G}$ e $J^{k} \mathcal{K}_{\Delta}$ coincidem. Portanto, as órbitas coincidem.

Passemos a verificar para que valores de $t$ podemos obter germes simples, para isto vamos trabalhar no espaço dos 2-jatos. Suponha que $h(0, z)$ é não degenerada (os outros casos seguem por adjacência). Podemos fazer mudanças de coordenadas para obtermos $h=\sum_{i=1}^{s} z_{i}^{2}+g(X)$. Considere o espaço afim $V$ dos 2-jatos dessa forma, lembrando que estamos classificando pelo grupo $J^{2} \mathcal{K}_{\Delta}$, temos que $V$ tem dimensão $\frac{[m(m+1)]^{2}+2 m(m+1)}{8}$ e o grupo que age tem dimensão $m^{2}$. Pela Observação 2.5.5, podemos obter germes simples somente se $m^{2} \geqslant$ $\frac{[m(m+1)]^{2}+2 m(m+1)}{8}$, ou seja, $8 m^{2} \geqslant m^{2}(m+1)^{2}+2 m(m+1)$, mas esta desigualdade só é verificada para $m=0$ ou $m=1$.

Assim, quando $t=n$ a classificação reduz a $\mathcal{K}$-classificação de funções. Quando $t=n-1$ a classificação se reduz à classificação de funções em variedades com bordo (com o $\mathcal{C}$ grupo presente). As singularidades simples estão determinadas por Arnold em [2] e [3].

Classificamos agora germes $A: \mathbb{C}^{r} \rightarrow \operatorname{Sim}_{n}(\mathbb{C})$, com $r=1$.

Proposição 3.1.4. Quando $r=1$, todos os germes $\mathcal{G}$-finitamente determinados são simples e $\mathcal{G}$-equivalentes a um germe da forma diag $\left(x^{m_{1}}, x^{m_{2}}, \ldots, x^{m_{n}}\right)$, onde $m_{1} \leqslant m_{2} \leqslant \ldots \leqslant m_{n}$. 
Este germe tem $\mathcal{G}$-codimens ão $\sum_{i=1}^{n}(n-i+1) m_{i}-1$.

Demonstração. Tomemos um germe finitamente determinado $A$. Se o primeiro jato de $A$ não nulo for o $k$-ésimo jato então $j^{k} A=x^{k} B$, onde $B$ é uma matriz constante. Pela Proposição 2.1.1, se $\operatorname{posto}(B)=s$ então podemos reduzir esse $k$-jato a $x^{k} I_{s}^{*} \oplus 0$.

Queremos determinar as órbitas em $J^{k+1}\left(\mathbb{C}, \operatorname{Sim}_{n}\right)$ de elementos cujo $k$-jato é igual a $x^{k} I_{s}^{*} \oplus 0$. Para isto, calculemos a transversal completa T. O espaço tangente à órbita de $J^{k+1} \mathcal{G}_{1}$. A em $A$, ou seja $T_{A} J^{k+1} \mathcal{G}_{1}$. $A$, contém todos os elementos de $H^{k+1} \mathcal{S}$ exceto os elementos $x^{k+1} E_{i j}$, em que $i$ é maior que s. De fato, quando $i \leqslant s$, tomando $\left(x^{k} I_{s}^{*} \oplus 0\right)_{j i}$ e multiplicando por $x$ temos $x\left(x^{k} I_{s}^{*} \oplus 0\right)_{j i} \in T_{A} J^{k+1} \mathcal{G}_{1}$. A, mas $x\left(x^{k} I_{s}^{*} \oplus 0\right)_{j i}=x^{k+1} E_{i j}$, o que implica $x^{k+1} E_{i j} \in$ $T_{A} J^{k+1} \mathcal{G} A$.

Logo, se $C \in \operatorname{Sim}_{n-s}(\mathbb{C})$ denota uma matriz qualquer, segue da Proposição 2.3.9 que $j^{k+1} A$ é $J^{k+1} \mathcal{G}$-equivalente a um elemento da forma $x^{k} I_{s}^{*} \oplus x^{k+1} C$. E o resultado segue por indução.

\subsection{Sobre o 1-jato de posto 1}

A seguir estudamos as formas do 1-jato $\alpha$ quando posto $(\alpha)=1$ e quais as possibilidades para $r, n$ para que $\alpha$ seja germe simples. Estes casos serão concluídos nos estudos das próximas seções, completando assim a classificação para posto $(\alpha)=1$.

Proposição 3.2.1. Suponha posto $(\alpha)=1$ então:

(1) Podemos reduzir o 1-jato à forma normal $x_{1} I_{t}^{*}$.

(2) Singularidades simples com 1-jato $x_{1} I_{t}^{*}$ pode acontecer somente se $n=1 ; r=1$; $(r, n)=(2,2)$

Demonstração. Para a prova de $(1)$, se posto $(\alpha)=1$ então podemos fazer mudanças de coordenadas na fonte de maneira que $\alpha$ seja equivalente à:

$$
\left(x_{1}, \ldots x_{r}\right) \mapsto x_{1} B
$$

onde $B \in \operatorname{Sim}_{n}$. Por outro lado, pela Proposição 2.1.1 podemos tomar elementos em $\mathcal{H}$ de maneira a obter $x_{1} I_{t}^{*}$.

Para (2), pela Proposição 2.5.8 singularidades simples podem ocorrer se $n=1,2$ ou $r=1$; $n=3$ e $r=2 ; n \geqslant 3$ com coposto $(\alpha)=0$ ou $1 ; n=3$ e posto $(\alpha)=4$. Porém os dois últimos dois casos não precisamos considerar pois estamos supondo posto $(\alpha)=1$. Desta forma, sobram os casos listados em (2) e os casos

- $n=2$ e $r \geqslant 3$

- $n=3$ e $r=2$. 
Considere o caso $n=2$ e $r \geqslant 3$ e suponha $t=2$. Usando a Proposição 2.3.9 segue que seu 2-jato é $J^{2} \mathcal{G}$-equivalente a um elemento da forma:

$$
\left(\begin{array}{cc}
x_{1} & g(y) \\
g(y) & x_{1}+f(y)
\end{array}\right), \text { onde } y=\left(x_{2}, \ldots, x_{r}\right) \text { e } f, g \in \mathcal{M}_{r-1}^{2} .
$$

Seu discriminante é dado por $x_{1}^{2}+x_{1} f(y)-g^{2}(y)=\left(x_{1}+\frac{f(y)}{2}\right)^{2}-\left(g^{2}(y)+\frac{f^{2}(y)}{4}\right)$. Fazendo a mudança de coordenadas $\left(x_{1}, x_{2}, \ldots, x_{r}\right) \rightarrow\left(x_{1}-\frac{f(y)}{2}, x_{2}, x_{3}, \ldots, x_{r}\right)$, obtemos:

$$
x_{1}^{2}-\left(g^{2}(y)+\frac{f^{2}(y)}{4}\right) \text {. }
$$

Sabemos que classificar esta função se reduz a classificar $\left(g^{2}(y)+\frac{f^{2}(y)}{4}\right)$, que é não simples (inicia com termos de grau 4). Portanto o 2-jato da matriz é não simples.

Quando $t<2$, basta usarmos o fato que toda matriz finitamente determinada com 1-jato $x_{1} I_{t}^{*}$ é adjacente a uma matriz com 1 -jato $x_{1} I_{2}^{*}$, que é não simples pelo que foi dito acima, logo, será não simples também.

Considere o caso $r=2$ e $n=3$, e suponha que $t=3$. Calculando sua 2-transversal completa $T$ segue que seu 2-jato será $J^{2} \mathcal{G}$-equivalente a um elemento da forma:

$$
\left(\begin{array}{ccc}
x & a y^{2} & b y^{2} \\
a y^{2} & x+c y^{2} & d y^{2} \\
b y^{2} & d y^{2} & x+e y^{2}
\end{array}\right) \text {, onde } a, b, c, d, e \in \mathbb{C} .
$$

Novamente seu discriminante possui uma singularidade não simples. O caso $t<3$, segue pelo mesmo argumento que $n=2$.

Pela Proposição 2.5.8, os possíveis valores para que o 1-jato $\alpha$ possa ser simples são:

- $n=1,2$ ou $r=1$;

- $r=2$ e $n=3$;

- para $n \geqslant 3$, coposto $(\alpha)=0$ ou 1 ;

- se $n=3$ então posto $(\alpha)=4$.

Quando $n=1$, temos coposto $(\alpha)=0,1$, que já foram tratados no Teorema 3.1.1. Quando $n=2$, temos coposto $(\alpha) \leqslant 3$. Os casos em que coposto $(\alpha)$ é 0 ou 1 , foram tratados no Teorema 3.1.1. Assim, resta o caso em que coposto $(\alpha)=2,3$, ou seja, os casos em que posto $(\alpha)=1,2$. Pela Proposição 3.2.1, temos que os 1-jatos de posto 1, e por adjacência os de posto 0, que podem ser simples são os casos em que $r=1$ ou $r=2$. Quando $n \geqslant 3$, $\operatorname{coposto}(\alpha)=0$ ou 1 , segue da Proposição 3.1.1.

Com os mesmos argumentos que usaremos para mostrar que um germe é não simples nas seções 3.3 e 3.4, podemos mostrar que quando $r>4$ não há germes simples no caso $n=3$ e posto $(\alpha)=4$. Assim, resta analisarmos os seguintes casos: 
- $r=n=2$;

- $(r, n)=(2,3)$;

- $n=3$ e $\operatorname{posto}(\alpha)=4$.

\subsection{Caso $\mathbf{r}=\mathbf{n}=2$}

Nesta seção classificamos germes simples quando $r=n=2$ e obtivemos o seguinte resultado:

Teorema 3.3.1. Quando $r=n=2$ as formas normais para os germes $\mathcal{G}$-simples são:

$$
\left(\begin{array}{cc}
y^{k} & x \\
x & y^{l}
\end{array}\right) ;\left(\begin{array}{cc}
x & 0 \\
0 & y^{2}+x^{k}
\end{array}\right) ;\left(\begin{array}{cc}
x & 0 \\
0 & x y+y^{k}
\end{array}\right) ;\left(\begin{array}{cc}
x & y^{k} \\
y^{k} & x y
\end{array}\right) ;\left(\begin{array}{cc}
x & y^{2} \\
y^{2} & x^{2}
\end{array}\right) ;\left(\begin{array}{cc}
x & 0 \\
0 & x^{2}+y^{3}
\end{array}\right) \text {. }
$$

com $k \geqslant 1$ e $l \geqslant 2$ para a primeira forma $e k \geqslant 2$ para as outras formas.

Identificando a matriz $A=\left(\begin{array}{ll}a & b \\ b & c\end{array}\right)$ com o vetor $(a, b, c)$, temos pela Proposição 2.3 .2 que seu $\mathcal{G}$-espaço tangente é gerado por $\left(a_{x}, b_{x}, c_{x}\right),\left(a_{y}, b_{y}, c_{y}\right),(2 a, b, 0),(2 b, c, 0),(0, a, 2 b)$, $(0, b, 2 c)$.

Pela Proposição 2.6.2, as órbitas dos 1-jatos pela ação de $J^{1} \mathcal{G}$ são:

$$
(x, y, x),(x, y, 0),(x, 0, x),(x, 0,0) \text { e }(0,0,0)
$$

Os dois primeiros 1-jatos acima possuem coposto 1 e assim já foram tratados na Proposição 3.1.3. A seguir vamos utilizar a Transversal Completa para obtermos as órbitas provenientes dos outros 1-jatos. Comecemos com:

Proposição 3.3.2. O 1-jato $(x, 0,0)$ gera as cinco últimas formas normais dadas no Teorema 3.3.1.

Demonstração. Considere $\alpha(x, y)=(x, 0,0)$. Queremos determinar as órbitas em $J^{2}\left(\mathbb{C}^{2}, \operatorname{Sim}_{2}(\mathbb{C})\right)$, pela ação de $J^{2} \mathcal{G}$, cujo 1-jato seja igual a $\alpha$. Para isto, calculando a transversal completa $T$, temos $T=\mathbb{R}\left\{\left(0, y^{2}, 0\right),\left(0,0, x^{2}\right),(0,0, x y),\left(0,0, y^{2}\right)\right\}$. Pela Proposição 2.3.9, segue que $j^{2} A$ é $J^{2} \mathcal{G}$-equivalente a $\left(x, a y^{2}, b_{0} x^{2}+b_{1} x y+b_{2} y^{2}\right)$. Para calcular as órbitas desta transversal em $J^{2}\left(\mathbb{C}^{2}, \operatorname{Sim}_{2}(\mathbb{C})\right)$, dividiremos nos seguintes casos:

- $b_{2} \neq 0$, então podemos reduzir $j^{2} A$ à $\left(x, 0, x^{2}+y^{2}\right)$ ou $\left(x, 0, y^{2}\right)$.

Com efeito, primeiro vamos mostrar que podemos reduzir $j^{2} A$ a $\left(x, 0, b_{0} x^{2}+\widetilde{b_{1}} x y+y^{2}\right)$,

onde $\widetilde{b_{1}}=\frac{b_{1}}{\sqrt{b_{2}}}$, independente dos valores de $b_{0}, b_{1}$. Considere $\phi(x, y)=\left(x, \frac{y}{\sqrt{b_{2}}}\right)$, temos que $\phi$ é um germe de difeomorfismo e compondo com $j^{2} A$ obtemos $\left(x, \widetilde{a} y^{2}, b_{0} x^{2}+\widetilde{b_{1}} x y+y^{2}\right)$. 
Para cada $b_{0}, \widetilde{b_{1}}$ fixo, vamos aplicar o Lema de Mather 1.1 .18 para $M=J^{2}\left(\mathbb{C}^{2}, \operatorname{Sim}_{2}(\mathbb{C})\right)$, $\mathrm{G}=J^{2} \mathcal{G}$ e $N=\left\{p \in J^{2}\left(\mathbb{C}^{2}, \operatorname{Sim}_{2}(\mathbb{C})\right) / p=\left(x, a y^{2}, b_{0} x^{2}+\widetilde{b_{1}} x y+y^{2}\right), a \in \mathbb{C}\right\}$. Temos que $N$ é uma variedade conexa e se $p \in N$ então $\mathrm{T}_{p} N=\mathbb{C}\left\{\left(0, y^{2}, 0\right)\right\}$. Por outro lado, temos que $\mathrm{T}_{p} \mathrm{G}_{p}$ é gerado por $\mathcal{M}\left\{\left(1,0,2 b_{0} x+b_{1} y\right) ;\left(0,2 a y, b_{1} x+2 y\right)\right\}+\mathcal{O}\left\{\left(2 x, a y^{2}, 0\right) ;\left(2 a y^{2}, b_{0} x^{2}+\right.\right.$ $\left.\left.b_{1} x y+y^{2}, 0\right) ;\left(0, x, 2 a y^{2}\right) ;\left(0, a y^{2}, 2 b_{0} x^{2}+2 b_{1} x y+2 y^{2}\right)\right\}$.

Lembrando que estamos em $J^{2}\left(\mathbb{C}^{2}, \operatorname{Sim}_{2}(\mathbb{C})\right)$, se multiplicarmos $\left(1,0,2 b_{0} x+b_{1} y\right)$ por $y^{2}$ obtemos $\left(y^{2}, 0,0\right)$ e multiplicando $\left(0, x, 2 a y^{2}\right)$ por $x$ e $y$ obtemos respectivamente $\left(0, x^{2}, 0\right)$ e $(0, x y, 0)$. Segue que

$$
\left(0, y^{2}, 0\right)=\left(2 a y^{2}, b_{0} x^{2}+b_{1} x y+y^{2}, 0\right)-2 a\left(y^{2}, 0,0\right)-b_{0}\left(0, x^{2}, 0\right)-b_{1}(0, x y, 0)
$$

ou seja, $\left(0, y^{2}, 0\right) \in \mathrm{T}_{p} \mathrm{G}_{p} \Rightarrow \mathrm{T}_{p} N \subset \mathrm{T}_{p} \mathrm{G}_{p}, \forall p \in N$, e como dim $\mathrm{T}_{p} G_{p}=$ constante, $\forall p \in N$, então as duas condições do Lema de Mather são satisfeitas o que implica que $N$ está contida em uma única órbita. Portanto podemos tomar $j^{2} A=\left(x, 0, b_{0} x^{2}+\widetilde{b_{1}} x y+y^{2}\right)$.

Usando $\psi(x, y)=\left(x, y-\frac{b_{1}}{2} x\right)$, e compondo com $j^{2} A$, temos que $j^{2} A$ é $J^{2} \mathcal{G}$-equivalente a $\left(x, 0, \widetilde{b_{0}} x^{2}+y^{2}\right)$. Se $\widetilde{b_{0}}=0$ então $j^{2} A$ é equivalente a $\left(x, 0, y^{2}\right)$ e se $\widetilde{b_{0}} \neq 0$, tomando

$$
\zeta(x, y)=\left(x, y \sqrt{\widetilde{b_{0}}}\right) \text { e } X=\left(\begin{array}{cc}
1 & 0 \\
0 & \frac{1}{\sqrt{\widetilde{b_{0}}}}
\end{array}\right) \Rightarrow X^{t}\left(j^{2} A \circ \zeta\right) X=\left(x, 0, x^{2}+y^{2}\right) .
$$

O germe $\left(x, 0, x^{2}+y^{2}\right)$ é 2-determinado. Com efeito seu $\mathcal{G}$-espaço tangente é dado por $\mathcal{M}\{(1,0,2 x) ;(0,0, y)\}+\mathcal{O}\left\{(x, 0,0) ;\left(0, x^{2}+y^{2}, 0\right) ;(0, x, 0) ;\left(0,0, x^{2}+y^{2}\right)\right\}$, podemos ver facilmente que $T \mathcal{G} j^{2} A \supset \mathcal{M}^{2} \mathcal{S}$. Logo, pela Proposição 2.4.3, temos que $\left(x, 0, x^{2}+y^{2}\right)$ é 2-determinado.

Para o germe $\left(x, 0, y^{2}\right)$, uma k-transversal completa é dada por $\left(x, 0, y^{2}+b x^{k}\right)$. Se $b \neq 0$ por mudanças escalares podemos reduzi-lo a $\left(x, 0, y^{2}+x^{k}\right)$, que é k-determinado.

- $b_{2}=0$ e $b_{1} \neq 0$, então $j^{2} A(x, y)=\left(x, a y^{2}, b_{0} x^{2}+b_{1} x y\right)$ e podemos reduzir à:

$$
(x, 0, x y) \text { ou }\left(x, y^{2}, x y\right) .
$$

Com efeito, se $a=0$, considere o germe $\phi(x, y)=\left(x, \frac{1}{b_{1}}\left(y-b_{0} x\right)\right)$, segue que $j^{2} A \circ \phi=$ $(x, 0, x y)$.

Agora se $a \neq 0$, considere $\varphi(x, y)=\left(\frac{a}{b_{1}^{2}} x, \frac{1}{b_{1}} y\right)$ e $X=\left(\begin{array}{cc}\frac{b_{1}}{\sqrt{a}} & 0 \\ 0 & \frac{b_{1}}{\sqrt{a}}\end{array}\right)$, então:

$$
X^{t}\left(j^{2} A \circ \varphi\right) X=\left(x, y^{2}, \widetilde{b_{0}} x^{2}+x y\right), \text { onde } \quad \widetilde{b_{0}}=\frac{a b_{0}}{b_{1}^{2}}
$$

Aplicando o Lema de Mather 1.1 .18 para $M=J^{2}\left(\mathbb{C}^{2}, \operatorname{Sim}_{2}(\mathbb{C})\right), \mathrm{G}=J^{2} \mathcal{G}$ e $N=\{p \in$ $\left.J^{2}\left(\mathbb{C}^{2}, \operatorname{Sim}_{2}(\mathbb{C})\right) / p=\left(x, y^{2}, b x^{2}+x y\right), b \in \mathbb{C}\right\}$. Temos que $N$ é conexa e $\mathrm{T}_{p} N=\mathbb{R}\left\{\left(0,0, x^{2}\right)\right\}$, $\forall p \in N$. Por outro lado, $\mathrm{T}_{p} \mathrm{G}_{p}$ é gerado por:

$\mathcal{M}\{(1,0, a x+y) ;(0,2 y, x)\}+\mathcal{O}\left\{\left(2 x, y^{2}, 0\right) ;\left(2 y^{2}, a x^{2}+x y, 0\right) ;\left(0, x, 2 y^{2}\right) ;\left(0, y^{2}, 2 a x^{2}+2 x y\right)\right\}$. 
Multiplicando $\left(0, x, 2 y^{2}\right) \in \mathrm{T}_{p} \mathrm{G}_{p}$ por $y$, obtemos $(0, x y, 0)$ e multiplicando $(0,2 y, x) \in \mathrm{T}_{p} \mathrm{G}_{p}$ por $x$, obtemos $\left(0,2 x y, x^{2}\right)$. Logo,

$$
\left(0,2 x y, x^{2}\right)-2(0, x y, 0)=\left(0,0, x^{2}\right) \in \mathrm{T}_{p} \mathrm{G}_{p} \Rightarrow \mathrm{T}_{p} N \subset \mathrm{T}_{p} \mathrm{G}_{p}, \forall p \in N
$$

Além disso, $\operatorname{dim} \mathrm{T}_{p} \mathrm{G}_{p}=11, \forall p \in N$. Segue do Lema de Mather que $N$ está contida em uma única órbita. Assim, se $a \neq 0$, podemos tomar $j^{2} A(x, y)=\left(x, y^{2}, x y\right)$, que é 2-determinado.

Uma k-transversal completa para $(x, 0, x y)$ é dada por $\left(x, c y^{k}, x y+d y^{k}\right)$. Se $d \neq 0$ então o germe é equivalente à $\left(x, 0, x y+y^{k}\right)$ que é k-determinado e tem codimensão $2 k$. Se $d=0$ mas $c \neq 0$ então o germe é equivalente a $\left(x, y^{k}, x y\right)$ que também é $k$-determinado mas tem codimensão $2 k+1$.

- $b_{2}=b_{1}=0$. Temos $j^{2} A(x, y)=\left(x, a y^{2}, b_{0} x^{2}\right)$ e as órbitas em $J^{2}\left(\mathbb{C}^{2}, \operatorname{Sim}_{3}(\mathbb{C})\right)$ desta transversal são:

$$
\left(x, y^{2}, x^{2}\right),\left(x, y^{2}, 0\right),\left(x, 0, x^{2}\right),(x, 0,0)
$$

Facilmente vemos que:

$$
\begin{aligned}
& a=0 \text { e } b_{0} \neq 0 \Rightarrow\left(x, 0, x^{2}\right) \\
& a=0 \text { e } b_{0}=0 \Rightarrow(x, 0,0) \\
& a \neq 0 \text { e } b_{0}=0 \Rightarrow\left(x, y^{2}, 0\right) \\
& a \neq 0 \text { e } b_{0} \neq 0 \Rightarrow\left(x, y^{2}, x^{2}\right)
\end{aligned}
$$

O germe $\left(x, y^{2}, x^{2}\right)$ é 2-determinado. Agora, considerando $j^{2} A(x, y)=\left(x, 0, x^{2}\right)$, verifiquemos os 3 -jatos cujo 2-jato é igual a $j^{2} A$. Calculando a transversal completa $T$ temos $T=\mathbb{R}\left\{\left(0, y^{3}, 0\right) ;\left(0,0, x y^{2}\right) ;\left(0,0, y^{3}\right)\right\}$. Segue da Proposição 2.3 .9 que $j^{3} A$ é $J^{3} \mathcal{G}$-equivalente a um elemento da forma $\left(x, c y^{3}, x^{2}+d_{0} x y^{2}+d_{1} y^{3}\right)$. Supondo $d_{1} \neq 0$ e considerando $\phi=\left(x, \frac{y}{\sqrt[3]{d_{1}}}\right)$, segue que

$$
j^{3} A \circ \phi=\left(x, \widetilde{c} y^{3}, x^{2}+\widetilde{d}_{0} x y^{2}+y^{3}\right), \text { onde } \widetilde{c}=\frac{c}{d_{1}} \text { e } \widetilde{d}_{0}=\frac{d_{0}}{d_{1}^{\frac{2}{3}}} .
$$

Aplicando o Lema de Mather 1.1 .18 para $\mathrm{G}=J^{3} \mathcal{G}, \quad M=J^{3}\left(\mathbb{C}^{2}, \operatorname{Sim}_{2}(\mathbb{C})\right)$ e $N=$ $\left\{p \in J^{3}\left(\mathbb{C}^{2}, \operatorname{Sim}_{2}(\mathbb{C})\right) / p=\left(x, c y^{3}, x^{2}+d_{0} x y^{2}+y^{3}\right), c, d \in \mathbb{C}\right\}$. Temos que $N$ é conexa e $\mathrm{T}_{p} N=\mathbb{R}\left\{\left(0, y^{3}, 0\right) ;\left(0,0, x y^{2}\right)\right\}$, para todo $p$ em $N$. Podemos mostrar que $\mathrm{T}_{p} N \subset \mathrm{T}_{p} \mathrm{G}_{p}$ e que $\operatorname{dimT}_{p} \mathrm{G}_{p}$ é constante para todo $p$ em $N$. Portanto, podemos tomar $\left(x, 0, x^{2}+y^{3}\right)$ que é 3 -determinado. Se $d_{1}=0$ resulta em germe não simples. Os 2-jatos $\left(x, y^{2}, 0\right) ;(x, 0,0)$ também conduzem a germes não simples.

Proposição 3.3.3. Germes cujo 1-jato é $(x, 0, x)$, possuem as formas normais $\left(y^{k}, x, y^{l}\right)$. 
Demonstração. Considere $B(x, y)=\left(\begin{array}{ll}x & 0 \\ 0 & x\end{array}\right)$, tomando $X=\left(\begin{array}{cc}1 & 1 \\ i & -i\end{array}\right)$, vemos que $B$ é equivalente a $\alpha(x, y)=\left(\begin{array}{ll}0 & x \\ x & 0\end{array}\right)$, que é mais conveniente para a nossa lista. Escrevendo $\alpha$ na forma vetorial, temos $\alpha(x, y)=(0, x, 0)$. Seu $\mathcal{G}_{1}$-espaço tangente é gerado por:

$$
\mathcal{M}^{2}\{(0,1,0)\}+\mathcal{M}\{(0, x, 0) ;(2 x, 0,0) ;(0,0,2 x) ;(0, x, 0)\}
$$

Calculando a 2-transversal completa $T$ temos que $T=\mathbb{R}\left\{\left(y^{2}, 0,0\right) ;\left(0,0, y^{2}\right)\right\}$. Pela Proposição 2.3.9, segue que qualquer 2-jato cujo 1 -jato é igual a $\alpha$ é $J^{2} \mathcal{G}$-equivalente a um elemento da forma $\left(a y^{2}, x, b y^{2}\right)$ e podemos reduzi-lo a um dos seguintes casos:

$$
\left(y^{2}, x, y^{2}\right) ;\left(0, x, y^{2}\right) ;(0, x, 0)
$$

- Temos que $\left(y^{2}, x, y^{2}\right)$ é 2-determinado. De fato, seu $\mathcal{G}$-espaço tangente é gerado por:

$$
\mathcal{M}\{(0,1,0) ;(2 y, 0,2 y)\}+\mathcal{O}\left\{\left(2 y^{2}, x, 0\right) ;\left(2 x, y^{2}, 0\right) ;\left(0, y^{2}, 2 x\right) ;\left(0, x, 2 y^{2}\right)\right\},
$$

e claramente $\mathcal{M}^{2} \mathcal{S} \subset T \mathcal{G} j^{2} A$. Pela Proposição 2.4.3 temos que esse germe é 2-determinado.

- Para $\left(0, x, y^{2}\right)$, uma k-transversal completa $T$ é dada por $T=\mathbb{R}\left\{\left(y^{k}, 0,0\right)\right\}$. Segue da Proposição 2.3.9 que $j^{k} A$ é $J^{k} \mathcal{G}$-equivalente a um elemento da forma $\left(a y^{k}, x, y^{2}\right)$. Se $a \neq 0$, podemos reduzir este germe a $\left(y^{k}, x, y^{2}\right)$, que é k-determinado.

- $(0, x, 0)$, sua 3-transversal $T$ é dada por $T=\mathbb{R}\left\{\left(y^{3}, 0,0\right) ;\left(0,0, y^{3}\right)\right\}$. Pelo processo acima podemos mostrar que as formas normais serão dadas por $\left(y^{k}, x, y^{l}\right)$.

\subsection{Caso $\mathrm{r}=2$ e $\mathrm{n}=3$}

Nessa seção estamos interessados em classificar germes de aplicações quando $r=2$ e $n=3$. Vamos usar a Proposição 2.6.4, que lista as formas normais dos 1-jatos neste caso, para obtermos o seguinte resultado:

Teorema 3.4.1. Quando $r=2$ e $n=3$, os germes $\mathcal{G}$-simples possuem as seguintes formas $\left(\begin{array}{ccc}y^{k} & x & 0 \\ x & y^{l} & 0 \\ 0 & 0 & y\end{array}\right),\left(\begin{array}{lll}0 & x & y \\ x & y & 0 \\ y & 0 & x^{2}\end{array}\right),\left(\begin{array}{ccc}0 & x & y \\ x & y & 0 \\ y & 0 & x y\end{array}\right),\left(\begin{array}{ccc}0 & x & y \\ x & y & 0 \\ y & 0 & x^{3}\end{array}\right),\left(\begin{array}{ccc}x & 0 & 0 \\ 0 & y & x \\ 0 & 0 & y^{2} \\ 0 & y & x \\ y^{2} & x & 0\end{array}\right)$.
$\left(\begin{array}{cl}x \\ x\end{array}\right)$, 
Pela Proposição 3.2.1-(2), sabemos que os germes de posto 1 e por adjacência os de posto 0 são não simples. Então resta verificarmos os 1-jatos com posto 2.

Segue da Proposição 2.6.4 que as órbitas dos 1-jatos de posto 2 pela ação de $J^{1} \mathcal{G}$ são:

$$
\begin{aligned}
\left(\begin{array}{lll}
y & x & 0 \\
x & y & 0 \\
0 & 0 & y
\end{array}\right), & \left(\begin{array}{lll}
0 & x & 0 \\
x & y & 0 \\
0 & 0 & y
\end{array}\right),\left(\begin{array}{lll}
0 & y & x \\
y & x & 0 \\
x & 0 & 0
\end{array}\right),\left(\begin{array}{lll}
0 & x & 0 \\
x & 0 & 0 \\
0 & 0 & y
\end{array}\right),\left(\begin{array}{lll}
x & 0 & 0 \\
0 & y & x \\
0 & x & 0
\end{array}\right), \\
& \left(\begin{array}{lll}
x & 0 & 0 \\
0 & y & 0 \\
0 & 0 & 0
\end{array}\right),\left(\begin{array}{lll}
0 & x & y \\
x & 0 & 0 \\
y & 0 & 0
\end{array}\right),\left(\begin{array}{lll}
x & y & 0 \\
y & 0 & 0 \\
0 & 0 & 0
\end{array}\right) .
\end{aligned}
$$

Proposição 3.4.2. Os três últimos 1-jatos produzem germes não simples. Nos casos restantes podemos reduzir à classificação dada no Teorema 3.4.1.

Demonstração. Os três últimos produzem determinantes iniciando com termos de grau quatro, que tem módulo. De fato, os 1-jatos são:

$$
\left(\begin{array}{lll}
x & 0 & 0 \\
0 & y & 0 \\
0 & 0 & 0
\end{array}\right) ;\left(\begin{array}{ccc}
0 & x & y \\
x & 0 & 0 \\
y & 0 & 0
\end{array}\right) ;\left(\begin{array}{ccc}
x & y & 0 \\
y & 0 & 0 \\
0 & 0 & 0
\end{array}\right)
$$

Seja $A$ um germe com 1-jato equivalente a $\alpha$. No caso em que $\alpha(x, y)=\left(\begin{array}{ccc}x & 0 & 0 \\ 0 & y & 0 \\ 0 & 0 & 0\end{array}\right)$, calculando uma 2-transversal completa $T$ segue que $j^{2} A$ é $J^{2} \mathcal{G}$-equivalente a um elemento da forma $\left(\begin{array}{ccc}x & 0 & a y^{2} \\ 0 & y & b x^{2} \\ a y^{2} & b x^{2} & c x^{2}+d x y+e y^{2}\end{array}\right)$. Claramente seu determinante inicia com termos de grau 4, que é um germe não simples. Os outros dois casos seguem análogo.

Passemos a classificar os casos restantes.

- Comecemos com o 1-jato $\alpha=\left(\begin{array}{lll}y & x & 0 \\ x & y & 0 \\ 0 & 0 & y\end{array}\right)$. O $\mathcal{G}$-espaço tangente de $\alpha$ é dado por: $\mathcal{M}\left\{\left(\begin{array}{lll}0 & 1 & 0 \\ 1 & 0 & 0 \\ 0 & 0 & 0\end{array}\right) ;\left(\begin{array}{ccc}1 & 0 & 0 \\ 0 & 1 & 0 \\ 0 & 0 & 1\end{array}\right)\right\}+\mathcal{O}\left\{\left(\begin{array}{ccc}2 y & x & 0 \\ x & 0 & 0 \\ 0 & 0 & 0\end{array}\right) ;\left(\begin{array}{ccc}2 x & y & 0 \\ y & 0 & 0 \\ 0 & 0 & 0\end{array}\right) ;\left(\begin{array}{ccc}0 & 0 & y \\ 0 & 0 & 0 \\ y & 0 & 0\end{array}\right) ;\right.$ $\left.\left(\begin{array}{ccc}0 & y & 0 \\ y & 2 x & 0 \\ 0 & 0 & 0\end{array}\right) ;\left(\begin{array}{ccc}0 & x & 0 \\ x & 2 y & 0 \\ 0 & 0 & 0\end{array}\right) ;\left(\begin{array}{ccc}0 & 0 & 0 \\ 0 & 0 & y \\ 0 & y & 0\end{array}\right) ;\left(\begin{array}{ccc}0 & 0 & y \\ 0 & 0 & x \\ y & x & 0\end{array}\right) ;\left(\begin{array}{ccc}0 & 0 & x \\ 0 & 0 & y \\ x & y & 0\end{array}\right) ;\left(\begin{array}{ccc}0 & 0 & 0 \\ 0 & 0 & 0 \\ 0 & 0 & y\end{array}\right)\right\}$ 
Podemos mostrar que ele é 1-determinado. Logo, qualquer elemento com esse 1-jato será equivalente a $\left(\begin{array}{ccc}y & x & 0 \\ x & y & 0 \\ 0 & 0 & y\end{array}\right)$

- Analisemos o 1-jato $\alpha=\left(\begin{array}{lll}0 & x & 0 \\ x & y & 0 \\ 0 & 0 & y\end{array}\right)$. Calculando a sua 2-transversal completa temos que $T=\mathbb{C}\left\{\left(\begin{array}{lll}y^{2} & 0 & 0 \\ 0 & 0 & 0 \\ 0 & 0 & 0\end{array}\right)\right\}$. Pela Proposição 2.3.9, $j^{2} A$ é $J^{2} \mathcal{G}$-equivalente a um elemento da forma $\left(\begin{array}{ccc}a y^{2} & x & 0 \\ x & y & 0 \\ 0 & 0 & y\end{array}\right)$. Se $a \neq 0$, tomando $X=\left(\begin{array}{ccc}1 / \sqrt{a} & 0 & 0 \\ 0 & 1 & 0 \\ 0 & 0 & 1\end{array}\right)$ e $\phi(x, y)=(\sqrt{a} x, y)$, segue que:

$$
X^{t}\left(j^{2} A \circ \phi\right) X=\left(\begin{array}{ccc}
y^{2} & x & 0 \\
x & y & 0 \\
0 & 0 & y
\end{array}\right), \text { que é 2-determinado. }
$$

Quando $a=0$, uma $k$-transversal completa será dada por $T=\mathbb{C}\left\{\left(\begin{array}{lll}y^{k} & 0 & 0 \\ 0 & 0 & 0 \\ 0 & 0 & 0\end{array}\right)\right\}$. Fazendo mudanças escalares, temos que seu $k$-jato será $J^{k} \mathcal{G}$-equivalente a um elemento da forma $\left(\begin{array}{ccc}y^{k} & x & 0 \\ x & y & 0 \\ 0 & 0 & y\end{array}\right)$, que é $k$-determinado.

- Considerando $\left(\begin{array}{lll}0 & y & x \\ y & x & 0 \\ x & 0 & 0\end{array}\right)$, temos que ele é $\mathcal{G}$-equivalente a $\alpha=\left(\begin{array}{lll}0 & x & y \\ x & y & 0 \\ y & 0 & 0\end{array}\right)$, que é mais conveniente para nossa lista. Calculando sua 2-transversal completa $T$, temos que $T=$ $\mathbb{C}\left\{\left(\begin{array}{ccc}0 & 0 & 0 \\ 0 & 0 & 0 \\ 0 & 0 & x y\end{array}\right) ;\left(\begin{array}{ccc}0 & 0 & 0 \\ 0 & 0 & 0 \\ 0 & 0 & x^{2}\end{array}\right)\right\}$. Segue da Proposição 2.3.9, que $j^{2} A$ é $J^{2} \mathcal{G}$-equivalente a um elemento da forma $\left(\begin{array}{ccc}0 & x & y \\ x & y & 0 \\ y & 0 & a x^{2}+b x y\end{array}\right)$. Temos três casos a considerar:

(1) $a \neq 0$. 
Tomando $X=\left(\begin{array}{ccc}1 & 0 & 0 \\ 0 & 1 / \sqrt{a} & 0 \\ 0 & 0 & 1 / a\end{array}\right)$ e $\phi(x, y)=(x \sqrt{a}, a y)$ segue que :

$$
X^{t}\left(j^{2} A \circ\right) X=\left(\begin{array}{ccc}
0 & x & y \\
x & y & 0 \\
y & 0 & x^{2}+\widetilde{b} x y
\end{array}\right) \text {, onde } \widetilde{b}=\frac{b \sqrt{a}}{a} .
$$

Vamos aplicar o Lema de Mather 1.1 .18 para $\quad G=J^{2} \mathcal{G}, \quad M=J^{2}\left(\mathbb{C}^{2}, \operatorname{Sim}_{3}(\mathbb{C})\right)$ e $N=\left\{A \in J^{2}\left(\mathbb{C}^{2}, \operatorname{Sim}_{3}(\mathbb{C})\right): A=\left(\begin{array}{lll}0 & x & y \\ x & y & 0 \\ y & 0 & x^{2}+b x y\end{array}\right) ; b \in \mathbb{C}\right\}$. Temos que $N$ é conexa e $T_{A} N=\mathbb{C}\left\{\left(\begin{array}{lll}0 & 0 & 0 \\ 0 & 0 & 0 \\ 0 & 0 & x y\end{array}\right)\right\}, \forall A \in N$. Por outro lado, tomando os elementos $A_{21}=$ $\left(\begin{array}{ccc}0 & 0 & 0 \\ 0 & 2 x & y \\ 0 & y & 0\end{array}\right)$ e $A_{32}=\left(\begin{array}{ccc}0 & 0 & x \\ 0 & 0 & y \\ x & y & 0\end{array}\right)$, ambos em $T_{A} G \cdot A$, segue que

$$
x A_{21}+2 x A_{32}=\left(\begin{array}{ccc}
0 & 0 & 2 x^{2} \\
0 & 2 x^{2} & 3 x y \\
2 x^{2} & 3 x y & 0
\end{array}\right) \in T_{A} G \cdot A
$$

Multiplicando $A_{y} \in T_{A} \mathcal{G}$.A por $x^{2}$ e lembrando que estamos em $J^{2} \mathcal{G}$, obtemos $\left(\begin{array}{ccc}0 & 0 & x^{2} \\ 0 & x^{2} & 0 \\ x^{2} & 0 & 0\end{array}\right) \in$ $T_{A} G$.A. Logo,

$$
\left(\begin{array}{ccc}
0 & 0 & 2 x^{2} \\
0 & 2 x^{2} & 3 x y \\
2 x^{2} & 3 x y & 0
\end{array}\right)-2\left(\begin{array}{ccc}
0 & 0 & x^{2} \\
0 & x^{2} & 0 \\
x^{2} & 0 & 0
\end{array}\right)=\left(\begin{array}{ccc}
0 & 0 & 0 \\
0 & 0 & 3 x y \\
0 & 3 x y & 0
\end{array}\right) \in T_{A} \mathcal{G} \cdot A .
$$

Tomando $A_{23}$ e $A_{31}$, e usando o elemento logo acima, segue que $\left(\begin{array}{ccc}0 & y & 0 \\ y & 0 & x^{2} \\ 0 & x^{2} & 0\end{array}\right)$, 


$$
\begin{aligned}
& \left(\begin{array}{ccc}
0 & 0 & 0 \\
0 & 0 & 0 \\
0 & 0 & y^{2}
\end{array}\right) \in T_{A} G . A \text {. Multiplicando } A_{x} \text { por } y \text {, temos: } \\
& \left(\begin{array}{ccc}
0 & y & 0 \\
y & 0 & 0 \\
0 & 0 & 2 x y+b y^{2}
\end{array}\right)-b\left(\begin{array}{ccc}
0 & 0 & 0 \\
0 & 0 & 0 \\
0 & 0 & y^{2}
\end{array}\right)-\left(\begin{array}{ccc}
0 & y & 0 \\
y & 0 & x^{2} \\
y & x^{2} & 0
\end{array}\right)=
\end{aligned}
$$

$$
\left(\begin{array}{ccc}
0 & 0 & 0 \\
0 & 0 & -x^{2} \\
0 & -x^{2} & 2 x y
\end{array}\right) \in T_{A} G \cdot A .
$$

Finalmente, multiplicando $A_{31}=\left(\begin{array}{ccc}0 & 0 & 0 \\ 0 & 0 & x \\ 0 & x & 2 y\end{array}\right)$ por $x$, segue que :

$$
\left(\begin{array}{ccc}
0 & 0 & 0 \\
0 & 0 & x^{2} \\
0 & x^{2} & 2 x y
\end{array}\right)+\left(\begin{array}{ccc}
0 & 0 & 0 \\
0 & 0 & -x^{2} \\
0 & -x^{2} & 2 x y
\end{array}\right)=\left(\begin{array}{ccc}
0 & 0 & 0 \\
0 & 0 & 0 \\
0 & 0 & 4 x y
\end{array}\right) \in T_{A} G . A .
$$

Portanto $T_{A} N \subset T_{A} G . A$ e ainda $\operatorname{dim} T_{A} G . A=28, \forall A \in N$. Como as duas condições do Lema de Mather 1.1.18 são satisfeitas segue que $N$ esta contida em uma única órbita.

Logo, $j^{2} A$ é equivalente a $\left(\begin{array}{ccc}0 & x & y \\ x & y & 0 \\ y & 0 & x^{2}\end{array}\right)$, que é 2-determinado.

(2) $a=0$ e $b \neq 0$.

Considerando $X=\left(\begin{array}{ccc}\sqrt{b} & 0 & 0 \\ 0 & \sqrt{b} & 0 \\ 0 & 0 & \sqrt{b}\end{array}\right)$ e $\phi(x, y)=\frac{1}{b}(x, y)$. Temos que $j^{2} A$ é equivalente a $X^{t}\left(j^{2} A \circ \phi\right) X=\left(\begin{array}{ccc}0 & x & y \\ x & y & 0 \\ y & 0 & x y\end{array}\right)$, que é finitamente determinado.

(3) $a=b=0$.

Calculando sua 3-transversal completa, temos que ela é dada por $T=\mathbb{C}\left\{\left(\begin{array}{ccc}0 & 0 & 0 \\ 0 & 0 & 0 \\ 0 & 0 & x^{2} y\end{array}\right),\left(\begin{array}{ccc}0 & 0 & 0 \\ 0 & 0 & 0 \\ 0 & 0 & x^{3}\end{array}\right)\right\}$. Segue da Proposição 2.3 .9 que $j^{3} A$ é $J^{3} \mathcal{G}$ equivalente a um elemento da forma $\left(\begin{array}{ccc}0 & x & y \\ x & y & 0 \\ y & 0 & c x^{2} y+d x^{3}\end{array}\right)$. Se $d \neq 0$, fazendo mudanças 
de coordenadas com $X=\left(\begin{array}{ccc}1 & 0 & 0 \\ 0 & 1 / d & 0 \\ 0 & 0 & 1 / d^{2}\end{array}\right)$ e $\phi(x, y)=\left(b x, b^{2} y\right)$ temos que $j^{3} A$ é equivalente a $\left(\begin{array}{ccc}0 & x & y \\ x & y & 0 \\ y & 0 & c x^{2} y+x^{3}\end{array}\right)$. Aplicando o Lema de Mather 1.1.18 para $G=J^{3} \mathcal{G}$ $M=J^{3}\left(\mathbb{C}^{2}, \operatorname{Sim}_{3}\right)$ e $N=\left\{A \in J^{3}\left(\mathbb{C}^{2}, \operatorname{Sim}_{3}\right): A=\left(\begin{array}{ccc}0 & x & y \\ x & y & 0 \\ y & 0 & x^{3}+c x^{2} y\end{array}\right) ; c \in \mathbb{C}\right\},$. Temos que $N$ é conexa e $T_{A} N=\mathbb{C}\left\{\left(\begin{array}{ccc}0 & 0 & 0 \\ 0 & 0 & 0 \\ 0 & 0 & x^{2} y\end{array}\right)\right\}, \forall A \in N$. Por outro lado,

$$
x A_{21}+2 x A_{32}-2 x^{2} A_{y}=\left(\begin{array}{ccc}
0 & 0 & 0 \\
0 & 0 & 3 x y \\
0 & 3 x y & 0
\end{array}\right) \in T_{A} G . A .
$$

$$
\begin{aligned}
& \text { Multiplicando } A_{31} \text { por } x^{2} \text {, obtemos }\left(\begin{array}{ccc}
0 & 0 & 0 \\
0 & 0 & x^{3} \\
0 & x^{3} & 2 x^{2} y
\end{array}\right) \in T_{A} G . A \text { e com } A_{23} \text { e (3.1), obtemos } \\
& \left(\begin{array}{ccc}
0 & y & 0 \\
y & 0 & x^{3} \\
0 & x^{3} & 0
\end{array}\right) \in T_{A} G . A, \log \left(\begin{array}{ccc}
0 & y & 0 \\
y & 0 & 0 \\
0 & 0 & -2 x^{2} y
\end{array}\right) \in T_{A} G . A \text {. Multiplicando } A_{31} \text { por } y \text { e } \\
& \text { usando (3.1), obtemos }\left(\begin{array}{ccc}
0 & 0 & 0 \\
0 & 0 & 0 \\
0 & 0 & y^{2}
\end{array}\right) \in T_{A} G . A \text {. Finalmente } \\
& y A_{x}-2 a x\left(\begin{array}{ccc}
0 & 0 & 0 \\
0 & 0 & 0 \\
0 & 0 & y^{2}
\end{array}\right)-\left(\begin{array}{ccc}
0 & y & 0 \\
y & 0 & 0 \\
0 & 0 & -2 x^{2} y
\end{array}\right)=\left(\begin{array}{ccc}
0 & 0 & 0 \\
0 & 0 & 0 \\
0 & 0 & 5 x^{2} y
\end{array}\right) \in T_{A} G . A
\end{aligned}
$$

Portanto $T_{A} N \subset T_{A} G \cdot A$ e $\operatorname{dim}_{A} G \cdot A=$ cte, $\forall A \in N$. Pelo Lema de Mather 1.1.18 segue que $N$ esta contida em uma uńica órbita e assim $j^{3} A$ é $J^{3} \mathcal{G}$-equivalente a $\left(\begin{array}{ccc}0 & x & y \\ x & y & 0 \\ y & 0 & x^{3}\end{array}\right)$, que é 3-determinado.

Quando $d=0$, seu determinante é $-y^{3}-c y x^{4}$, que é não simples. Esse módulo claramente pode ser realizado nas matrizes. Portanto, quando $d=0$ produz germe não simples. 
- Seja agora $\alpha=\left(\begin{array}{ccc}0 & x & 0 \\ x & 0 & 0 \\ 0 & 0 & y\end{array}\right)$. Calculando a 2-transversal completa $T$ vemos que ela pode ser dada por $T=\mathbb{C}\left\{\left(\begin{array}{ccc}y^{2} & 0 & 0 \\ 0 & 0 & 0 \\ 0 & 0 & 0\end{array}\right) ;\left(\begin{array}{ccc}0 & 0 & 0 \\ 0 & y^{2} & 0 \\ 0 & 0 & 0\end{array}\right)\right\}$. Segue da Proposição 2.3.9 que seu 2-jato será $J^{2} \mathcal{G}$-equivalente a um elemento da forma $\left(\begin{array}{ccc}a y^{2} & x & 0 \\ x & b y^{2} & 0 \\ 0 & 0 & y\end{array}\right)$.

(1) Se $a \neq 0$ e $b \neq 0$, tomando $X=\left(\begin{array}{ccc}\frac{1}{\sqrt{a}} & 0 & 0 \\ 0 & \frac{1}{\sqrt{b}} & 0 \\ 0 & 0 & 1\end{array}\right)$ e $\phi(x, y)=\left(\frac{x}{a b}, y\right)$, obtemos $\left(\begin{array}{ccc}y^{2} & x & 0 \\ x & y^{2} & 0 \\ 0 & 0 & y\end{array}\right)$, que é 2-determinado.

(2) Se $a \neq 0$ e $b=0$, podemos reduzir a $\left(\begin{array}{ccc}y^{2} & x & 0 \\ x & 0 & 0 \\ 0 & 0 & y\end{array}\right)$ e uma $k$-transversal completa para este elemento é dada por $\left(\begin{array}{ccc}y^{2} & x & 0 \\ x & c y^{k} & 0 \\ 0 & 0 & y\end{array}\right)$. Para $c \neq 0$ ele é $k$-determinado.

(3) Quando $a=0$ e $b \neq 0$ tomando $X=\left(\begin{array}{lll}0 & 1 & 0 \\ 1 & 0 & 0 \\ 0 & 0 & 1\end{array}\right)$, temos $X^{t}\left(j^{2} A\right) X=\left(\begin{array}{ccc}b y^{2} & x & 0 \\ x & 0 & 0 \\ 0 & 0 & y\end{array}\right)$, que pode ser reduzido a $\left(\begin{array}{ccc}y^{2} & x & 0 \\ x & 0 & 0 \\ 0 & 0 & y\end{array}\right)$ que coincide com o caso $a \neq 0$ e $b=0$, feito acima. Quando $a=0$ e $b=0$, uma 3-transversal completa é dada por $\left(\begin{array}{ccc}e y^{3} & x & 0 \\ x & f y^{3} & 0 \\ 0 & 0 & y\end{array}\right)$. Seguindo os mesmos argumentos acima, podemos mostrar que uma forma normal para este elemento é da forma $\left(\begin{array}{ccc}y^{k} & x & 0 \\ x & y^{l} & 0 \\ 0 & 0 & y\end{array}\right)$. 
- Já o 1-jato $\left(\begin{array}{ccc}x & 0 & 0 \\ 0 & y & x \\ 0 & x & 0\end{array}\right)$, fornece os germes simples $\left(\begin{array}{ccc}x & 0 & y^{2} \\ 0 & y & x \\ y^{2} & x & 0\end{array}\right)$ e $\left(\begin{array}{ccc}x & 0 & 0 \\ 0 & y & x \\ 0 & x & y^{2}\end{array}\right)$.

\subsection{Caso $r=4$ e $n=3$}

Nesta seção, vamos classificar germes de aplicações quando $r=4, n=3$ e posto $(\alpha)=4$. Usando a Proposição 2.6.5, que lista as formas normais dos 1-jatos deste caso, obtemos o seguinte resultado:

Teorema 3.5.1. Quando $r=4$ e $n=3$, os germes $\mathcal{G}$-simples possuem as seguintes formas $\left(\begin{array}{ccc}x & 0 & z \\ 0 & y+x^{k} & w \\ z & w & -y\end{array}\right)(k \geqslant 1),\left(\begin{array}{ccc}x & w^{2} & y \\ w^{2} & -y & z \\ y & z & w\end{array}\right),\left(\begin{array}{ccc}x & w z & y \\ w z & -y & z \\ y & z & w\end{array}\right) e\left(\begin{array}{ccc}x & w^{3} & y \\ w^{3} & -y & z \\ y & z & w\end{array}\right)$.

A Proposição 2.6.5 nos fornece as formas normais dos 1-jatos de posto 4 que são dados por:

$$
\begin{aligned}
\left(\begin{array}{ccc}
x & 0 & z \\
0 & y & w \\
z & w & -x-y
\end{array}\right), & \left(\begin{array}{ccc}
x & 0 & z \\
0 & y & w \\
z & w & -y
\end{array}\right),\left(\begin{array}{ccc}
x & 0 & y \\
0 & -y & z \\
y & z & w
\end{array}\right),\left(\begin{array}{ccc}
x & 0 & z \\
0 & y & w \\
z & w & 0
\end{array}\right),\left(\begin{array}{ccc}
x & z & w \\
z & 0 & -x \\
w & -x & y
\end{array}\right), \\
& \left(\begin{array}{ccc}
0 & y & z \\
y & 0 & w \\
z & w & x
\end{array}\right),\left(\begin{array}{ccc}
x & 0 & 0 \\
0 & y & w \\
0 & w & z
\end{array}\right),\left(\begin{array}{ccc}
0 & 0 & z \\
0 & x & w \\
z & w & y
\end{array}\right) .
\end{aligned}
$$

Usando os 1-jatos acima vamos aplicar o método da Transversal Completa para encontrarmos as formas normais dos germes simples em cada caso.

Proposição 3.5.2. O germe do 1-jato $\left(\begin{array}{ccc}x & 0 & z \\ 0 & y & w \\ z & w & 0\end{array}\right)$, contém os quatros últimos 1-jatos em seu fecho e não há 2-jato simples com este 1-jato.

Demonstração. Mostremos inicialmente que qualquer 2-jato com este 1-jato é não simples. Aplicando a Proposição 2.3.9 segue que qualquer elemento com esse 1-jato é $J^{2} \mathcal{G}$-equivalente a um elemento da forma $\left(\begin{array}{ccc}x & 0 & z \\ 0 & y & w \\ z & w & f(x, y)\end{array}\right)$, onde $f(x, y)=a_{1} x^{2}+a_{2} x y+a_{3} y^{2}$. Para mostrarmos o resultado basta mostrarmos que qualquer 2-jato desta forma é não simples. 
Aplicando a Proposição 2.5.4 para $\quad V=J^{2}\left(\mathbb{C}^{4}, \operatorname{Sim}_{3}(\mathbb{C})\right), \quad G=J^{2} \mathcal{G} \quad$ e $\quad W=$ $\left\{A \in J^{2}\left(\mathbb{C}^{4}, \operatorname{Sim}_{3}(\mathbb{C})\right) ; A=\left(\begin{array}{ccc}x & 0 & z \\ 0 & y & w \\ z & w & a_{1} x^{2}+a_{2} x y+a_{3} y^{2}\end{array}\right), a_{i} \in \mathbb{C}\right\}$.

Temos que $T_{w} W$ é gerado por $\left\{\left(\begin{array}{ccc}0 & 0 & 0 \\ 0 & 0 & 0 \\ 0 & 0 & x^{2}\end{array}\right) ;\left(\begin{array}{ccc}0 & 0 & 0 \\ 0 & 0 & 0 \\ 0 & 0 & x y\end{array}\right) ;\left(\begin{array}{ccc}0 & 0 & 0 \\ 0 & 0 & 0 \\ 0 & 0 & y^{2}\end{array}\right)\right\}$,

$\forall w \in W$. Facilmente mostra-se que $\left\{w \in W ; T_{w} W \subset T_{w} G \cdot w\right\}=\emptyset, \forall w \in W$. Pela Proposição 2.5.4, dado $w \in W$ então toda vizinhança $U$ de $w$ encontra um número infinito de $J^{2} \mathcal{G}$-órbitas. Portanto, qualquer 2-jato com 1-jato igual a $\left(\begin{array}{ccc}x & 0 & z \\ 0 & y & w \\ z & w & 0\end{array}\right)$ é não simples.

Resta mostrarmos que este germe contém os quatros últimos 1-jatos em seu fecho. Trabalhemos com os pencils relacionados com esses 1-jatos (Proposição 2.6.5). Tomando por exemplo o pencil $\left(x^{2}+2 y z, y^{2}\right)$ e a perturbação $\left(x^{2}+\epsilon x z+2 y z, y^{2}\right)$, para $\epsilon$ suficientemente pequeno e diferente de zero, podemos mostrar que ele é equivalente a $\left(x y, z^{2}\right)$. Os outros casos seguem analogamente.

Proposição 3.5.3. Os três 1-jatos restantes nos fornecem germes simples e suas formas normais são as do Teorema 3.5.1.

Demonstração. Usando a Proposição 2.4.3, temos que o 1-jato $\left(\begin{array}{ccc}x & 0 & z \\ 0 & y & w \\ z & w & -x-y\end{array}\right)$ é 1 determinado. Por conveniência tomaremos como forma normal para este germe o elemento $\left(\begin{array}{ccc}x & 0 & z \\ 0 & y+x & w \\ z & w & -y\end{array}\right)$

Analisemos o 1-jato $\left(\begin{array}{ccc}x & 0 & z \\ 0 & y & w \\ z & w & -y\end{array}\right)$. Calculando sua $k$-transversal completa $T$ segue que qualquer $k$-jato deste elemento é $J^{k} \mathcal{G}$-equivalente a $\left(\begin{array}{ccc}x & 0 & z \\ 0 & y+a x^{k} & w \\ z & w & -y\end{array}\right)$. Mudanças de coordenadas reduzem esse $k$-jato a $\left(\begin{array}{ccc}x & 0 & z \\ 0 & y & w \\ z & w & -y\end{array}\right)$ ou a $\left(\begin{array}{ccc}x & 0 & z \\ 0 & y+x^{k} & w \\ z & w & -y\end{array}\right)$. Sendo que 


$$
\left(\begin{array}{ccc}
x & 0 & z \\
0 & y+x^{k} & w \\
z & w & -y
\end{array}\right) \text { é } k \text {-determinado. }
$$

Trabalhemos agora com o 1-jato $\alpha=\left(\begin{array}{ccc}x & 0 & y \\ 0 & -y & z \\ y & z & w\end{array}\right)$. Aplicando a Proposição 2.3.9 segue que $j^{2} A$ é $J^{2} \mathcal{G}$-equivalente a um elemento da forma $\left(\begin{array}{ccc}x & a w^{2}+b w z & y \\ a w^{2}+b w z & -y & z \\ y & z & w\end{array}\right)$. Se $a \neq 0$, mudanças de coordenadas reduzem esse elemento a $\left(\begin{array}{ccc}x & w^{2}+\widetilde{b} w z & y \\ w^{2}+\widetilde{b} w z & -y & z \\ y & z & w\end{array}\right)$, onde $\widetilde{b}=\frac{b}{a}$. Aplicando o Lema de Mather 1.1 .18 para $M=J^{2}\left(\mathbb{C}^{4}, \operatorname{Sim}_{3}\right), G=J^{2} \mathcal{G}$ e $N=\left\{A \in J^{2}\left(\mathbb{C}^{4}, \operatorname{Sim}_{3}\right): A=\left(\begin{array}{ccc}x & w^{2}+b w z & y \\ w^{2}+b w z & -y & z \\ y & z & w\end{array}\right), b \in \mathbb{C}\right\}$, verifica-se que as duas condições do Lema de Mather são satisfeitas e $\operatorname{assim} j^{2} A$ é $J^{2} \mathcal{G}$-equivalente a $\left(\begin{array}{ccc}x & w^{2} & y \\ -y & z \\ w^{2} & z & w\end{array}\right)$, que é 2-determinado. Quando $a=0$ e $b \neq 0$, mudanças de coordenadas reduzem $j^{2} A$ a $\left(\begin{array}{ccc}x & w z & y \\ w z & -y & z \\ y & z & w\end{array}\right)$, que é 2-determinado. O caso em que $a=b=0$, nos fornece o germe simples $\left(\begin{array}{ccc}x & w^{3} & y \\ w^{3} & -y & z \\ y & z & w\end{array}\right)$. 


\section{Capítulo 4}

\section{Geometria dos germes simples}

Nesta seção estudamos a geometria complexa de germes simples classificados no Capítulo 3. Apresentamos alguns resultados sobre o criminante de um dado germe, fornecemos desdobramentos versais de alguns germes e estudamos com mais detalhe o caso $r=n=2$.

Durante o texto, faremos uso de alguns nomes e notações bastante conhecidos na teoria de singularidades que podem ser vistos em [24]. Dentre essas notações, as que nós mais utilizamos é a lista das formas normais para germes $\mathcal{R}$-simples de funções e suas respectivas nomenclaturas. Considerando $f: \mathbb{C}^{n} \rightarrow \mathbb{C}$ e $\left(x, y, x_{3}, \ldots, x_{n}\right)$ as coordenadas de um elemento arbitrário de $\mathbb{C}^{n}$, temos:

$$
\begin{array}{ll}
\text { Símbolo } & \text { Forma normal } \\
A_{k}(k \geqslant 1) & \pm x^{k+1}+q\left(y, x_{3}, \ldots, x_{n}\right) \\
D_{k}(k \geqslant 4) & x y^{2} \pm x^{k-1}+q\left(x_{3}, \ldots, x_{n}\right) \\
E_{6} & x^{3} \pm y^{4}+q\left(x_{3}, \ldots, x_{n}\right) \\
E_{7} & x^{3} \pm x y^{3}+q\left(x_{3}, \ldots, x_{n}\right) \\
E_{8} & x^{3} \pm y^{5}+q\left(x_{3}, \ldots, x_{n}\right),
\end{array}
$$

onde $q$ é uma forma quadrática não degenerada.

Dado um germe $A:\left(\mathbb{K}^{r}, 0\right) \rightarrow \operatorname{Sim}_{n}(\mathbb{K})$, definimos os seguintes elementos associados a este germe:

Definição 4.0.4. $O$ discriminante de um elemento $A \in \mathcal{S}$ é o conjunto

$$
D(A)=\left\{x \in \mathbb{K}^{r} ; \operatorname{det}(A(x))=0\right\} .
$$

Definição 4.0.5. Dado um elemento $A \in \mathcal{S}$, a variedade associada de $A$ é definida pelo conjunto

$$
M(A)=\left\{(x, v) \in \mathbb{K}^{r} \times \mathbb{K} P^{n-1} ; v^{t} A(x) v=0\right\} .
$$

Escrevemos $M(A)(x)$ para a quadrática associada a $A(x)$.

Definição 4.0.6. O criminante de $A, C(A)$, é definido como sendo o fecho do conjunto $\left\{(x, v) \in \mathbb{K}^{r} \times \mathbb{K} P^{n-1} ; A(x) v=0\right.$ e posto $\left.(A(x))=n-1\right\}$. 
Proposição 4.0.7. Os discriminante, criminante e a variedade associada são todos $\mathcal{G}$-invariantes no sentido natural.

\subsection{Singularidades do discriminante e número de Milnor}

O criminante de um dado germe possui a seguinte propriedade:

Proposição 4.1.1. Se $A:\left(\mathbb{C}^{r}, 0\right) \rightarrow \operatorname{Sim}_{n}$ é $\mathcal{G}$-finitamente determinado então $C(A)$ é o germe de uma variedade (ao longo de $0 \times \mathbb{C} P^{n-1}$ ) de dimensão $r-1$ e é suave fora de $0 \times \mathbb{C} P^{n-1}$.

Observação 4.1.2. Pela Proposição 4.1.1 temos que $C(A)$ é suave fora de $0 \times \mathbb{C} P^{n-1}$. Logo, suas singularidades podem ocorrer somente ao longo de $0 \times \mathbb{C} P^{n-1}$.

Exemplo 4.1.3. Considere $A:\left(\mathbb{C}^{2}, 0\right) \rightarrow \operatorname{Sim}_{2}(\mathbb{C})$, dado por $A(x, y)=\left(\begin{array}{cc}x & y \\ y & x^{2}\end{array}\right)$. Seu discriminante é dado por $\delta=x^{3}-y^{2}=0$, que tem uma singularidade $A_{2}$ em $(0,0)$. Temos que $\delta$ pode ser parametrizado por $\left(t^{2}, t^{3}\right)$ e o núcleo da correspondente matriz $A\left(t^{2}, t^{3}\right)$ é então gerado por $(-t, 1)$. Assim, se tomarmos a carta $q=1 \mathrm{em}[p, q] \in \mathbb{C} P^{1}$, o criminante é dado por $\left(t^{2}, t^{3},-t\right)$, que é equivalente a uma curva suave.

O exemplo abaixo nos mostra que as singularidades em $C(A)$ de um germe $A \in \mathcal{S}$ não necessariamente precisam ser isoladas, mesmo com a hipótese que $A$ é finitamente determinado.

Exemplo 4.1.4. Considere $A:\left(\mathbb{C}^{3}, 0\right) \rightarrow \operatorname{Sim}_{2}(\mathbb{C})$, dada por

$$
\left(\begin{array}{cc}
x^{2}-y^{2} & z^{2} \\
z^{2} & x^{2}+y^{2}
\end{array}\right)
$$

Este é um germe de $\mathcal{G}$-codimensão finita, $\log$ $\mathcal{G}$-finitamente determinado. Provemos que todo ponto de $0 \times \mathbb{C} P^{1}$ está em $C(A)$. De fato, dado $v=(p, q) \neq(0,0)$ podemos resolver as equações $\left(x^{2}-y^{2}\right) p+z^{2} q=z^{2} p+\left(x^{2}+y^{2}\right) q=0$, dadas por $A(x) v=0$, para algum $\left(x_{0}, y_{0}, z_{0}\right) \neq(0,0,0)$, assim $\left[\left(x_{0}, y_{0}, z_{0}\right) ;(p, q)\right] \in C(A)$. Como as equações são homogêneas em $(x, y, z)$, o ponto $t\left(x_{0}, y_{0}, z_{0}\right)$ é também uma solução, para todo $t$. Assim todo ponto de $0 \times \mathbb{C} P^{1}$ está em $C(A)$ uma vez que está no fecho destas soluções.

Tomando a carta $p=1$ para $\mathbb{C} P^{1}$, temos que o conjunto

$$
\left(x^{2}-y^{2}\right)+z^{2} q=z^{2}+\left(x^{2}+y^{2}\right) q=0
$$

possui uma linha de singularidades ao longo do eixo $q$. Com efeito, considerando $f_{1}=\left(x^{2}-\right.$ $\left.y^{2}\right)+z^{2} q$ e $f_{2}=z^{2}+\left(x^{2}+y^{2}\right) q$, temos: 


$$
\frac{\partial f_{i}}{\partial x}(0,0,0, q)=\frac{\partial f_{i}}{\partial y}(0,0,0, q)=\frac{\partial f_{i}}{\partial z}(0,0,0, q)=\frac{\partial f_{i}}{\partial q}(0,0,0, q)=0, \forall q \in \mathbb{C} \text { e } i=1,2 .
$$

Portanto, vemos que o criminante não necessariamente precisa ter singularidades isoladas ao longo de $0 \times \mathbb{C} P^{n-1}$.

Passemos a investigar em que condições o criminante de um germe $A \in \mathcal{S}$ possui singularidade isolada ao longo de $0 \times \mathbb{C} P^{n-1}$. Escrevendo o 1-jato de $A$ como $\sum x_{i} A_{i}$, com $A_{i} \in \operatorname{Sim}_{n}(\mathbb{C})$, prova-se que:

Proposição 4.1.5. Temos singularidades isoladas no Criminante se a seguinte afirmação se verificar: O conjunto $\left\{v=\left(v_{1}, \ldots, v_{n}\right) /\left[A_{1} v, \ldots, A_{r} v\right]\right.$ tem posto $\left.<n\right\}$ é um número finito de pontos em $\mathbb{C} P^{n-1}$. Em particular $r \geqslant n$.

Demonstração. Considerando as equações dadas por $A(x) v=0$ e diferenciando em relação a $x_{j}$ obtemos a matriz $\left[A_{1} v, \ldots, A_{r} v\right]$. Quando esta matriz possuir posto $n$ não temos singularidade em $(0, v) \in C(A)$. Logo, se o conjunto $\left\{v=\left(v_{1}, \ldots, v_{n}\right) /\left[A_{1} v, \ldots, A_{r} v\right]\right.$ tem posto $<n\}$ é dado por um número finito de pontos em $\mathbb{C} P^{n-1}$ então as singularidades em $C(A)$ são isoladas.

Exemplo 4.1.6. Considere o caso $n=2$ e suponha que $j^{1} A=\sum x_{i} A_{i}$. Nós temos singularidade não isolada somente se a matriz $2 \times 2 r$ dada por $\left[A_{1}, \ldots, A_{r}\right]$ tem posto $\leqslant 1$. Então neste caso temos o 1-jato nulo ou equivalente a

$$
\left(\begin{array}{cc}
x_{1} & 0 \\
0 & 0
\end{array}\right)
$$

Em todos os outros casos nós temos apenas singularidades isoladas.

Agora, suponha $n=2$ e que temos a forma geral:

$$
\left(\begin{array}{cc}
y & z \\
z & f(x, y)
\end{array}\right)
$$

onde $(x, y) \in \mathbb{C}^{r-2} \times \mathbb{C}$ e $f$ é uma função em uma variedade com bordo $y=0$ (Teorema 3.1.1-(2)(ii) ). Consideremos o caso em que $f$ tem uma singularidade simples isolada na origem. O criminante deste germe é dado por

$$
C(A)=\left\{(x, y, z,[p, q]) \in \mathbb{C}^{r} \times \mathbb{C} P^{1}, p y+q z=p z+q f(x, y)=0\right\} .
$$

Tomando a carta afim $p=1$ em $\mathbb{C} P^{1}$, obtemos

$$
\left\{\begin{array}{l}
y+q z=0 \\
z+q f(x,-q z)=0
\end{array}\right.
$$

que é suave. Por outro lado, tomando $q=1$ temos

$$
\left\{\begin{array}{l}
z+p y=0 \\
-p^{2} y+f(x, y)=0
\end{array}\right.
$$


Mostremos que a variedade $C(A)$ tem uma singularidade isolada em $(0,0,0,[0: 1])$ se $f$ é finitamente $\mathcal{R}_{\delta}$-determinada, isto é, $\operatorname{dim} \mathcal{O}_{2}\left\langle f_{1}, . ., f_{r-2}, y f_{y}\right\rangle<\infty$, onde $f_{i}$ é a derivada parcial de $f$ com relação a $x_{i}$. De fato, diferenciando $-p^{2} y+f(x, y)$ com respeito a $x_{i}, y, p$ e igualando os resultados a zero, obtemos $f_{x_{i}}=f_{y}-p^{2}=p y=0$. Se $p=0$ então $f_{x_{i}}=0$ e $f_{y}=0$. Como estamos supondo $f$ com singularidade isolada segue que $x=y=0$. Por outro lado, se $y=0$ então como a restrição de $f$ a $y=0$ tem uma singularidade isolada na origem nós deduzimos que $x=0$ e $p=0$ novamente. Portanto, o único ponto singular de $C(A)$ é $x=y=z=p=0$.

Obtemos abaixo um resultado que relaciona o número de Milnor $\mu$ de uma variedade de objetos quando $r=2$. No que segue nós estamos supondo que $f$ e $f(x, 0)$ têm singularidade isolada na origem.

Proposição 4.1.7. (1) Para qualquer $f$ nós temos $\mu(C)=\mu(f)+2 \mu(f(x, 0))$.

(2) Se $f$ é quase homogênea $\mu(D)=\mu(f)+2 \mu(f(x, 0))+1$. Em particular $\mu(C)=\mu(D)-1$.

Aplicaremos estes resultados em algumas singularidades simples.

Exemplo 4.1.8. (1) Seja $f(x, y)=x^{2}+y^{k}$ (tipo $\left.B_{k}\right)$. Temos que $\mu(f)=k-1$ e $\mu(f(x, 0))=$ 1 , então pela proposição acima temos $\mu(C)=k+1$ e $\mu(D)=k+2$. De fato, o discriminante de $(y, z, f)$ é dado por

$$
\delta=x^{2} y+y^{k+1}-z^{2}=0,
$$

que tem uma singularidade em $(0,0,0)$ do tipo $D_{k+2}$ então $\mu(D)=k+2$. Já as singularidades de $C(A)$, pelo que foi dito acima, são dadas por $-p^{2} y+f(x, y)=-p^{2} y+y^{k}+x^{2}=0$, que tem somente uma singularidade em $(0,0,0,[0,1])$ do tipo $D_{k+1}$ e assim $\mu(C)=k+1$.

(2) Seja $f(x, y)=x y+x^{k}\left(\right.$ tipo $\left.C_{k}\right)$. Temos $\mu(f)=1$ e $\mu(f(x, 0))=k-1$. $O$ discriminante de $\left(y, z, x y+x^{k}\right)$ é dado por

$$
\delta=x y^{2}+y x^{k}-z^{2}=0 .
$$

A mudança de coordenadas $(x, y, z) \mapsto\left(x, y-\frac{x^{k-1}}{2}, z\right)$, reduz este germe à $x y^{2}-\frac{x^{2 k-1}}{4}-z^{2}$, que é equivalente a $x y^{2}-x^{2 k-1}-z^{2}$. Portanto o discriminante possui uma singularidade do tipo $D_{2 k}$ em $(0,0,0)$. Analisemos o germe $-p^{2} y+x y+x^{k}$. Temos que sua singularidade é no ponto $(0,0,0)$ e com a mudança de coordenadas $(x, y, p) \rightarrow\left(x+p^{2}, y, p\right)$, obtemos $x y+\left(x+p^{2}\right)^{k}$. Mudanças de coordenadas na variável y reduzem este germe a $x y+p^{2 k}$, que é equivalente a $x^{2}-y^{2}+p^{2 k}$. Portanto $-p^{2} y+x y+x^{k}$ possui uma singularidade $A_{2 k-1}$

(3) Seja $f(x, y)=y^{2}+x^{3}$ (tipo $\left.F_{4}\right)$. O discriminante de $\left(y, z, y^{2}+x^{3}\right)$ é dado por $\delta=$ $y^{3}+y x^{3}-z^{2}=0$, que tem uma singularidade do tipo $E_{7}$. Já a singularidade do criminante é dada por $-p^{2} y+y^{2}+x^{3}$. A mudança de coordenadas $(x, y, p) \rightarrow\left(x, y+\frac{p^{2}}{2}, p\right)$ e mudanças escalares reduzem este germe a $x^{3}-p^{4}+y^{2}$. Portanto, $C(A)$ tem uma singularidade do tipo $E_{6}$ no ponto $(0,0,0,[0,1])$. 
(4) Esse é um exemplo bastante atipico. Seja $f(x, y)=y+x^{k}$. Temos que $f$ é não singular e $f(x, 0)$ tem uma singularidade isolada do tipo $A_{k-1}$ na origem, logo $\mu(f)=0$ e $\mu(f(x, 0))=k-1$. O discriminante do germe $(y, z, f)$ é dado por

$$
\delta=y^{2}+y x^{k}-z^{2}=0,
$$

que tem uma singularidade isolada na origem. Podemos ver que a hessiana de $\delta$ possui coposto 1 e $\mu(\delta)=2 k-1$. Logo, sua singularidade é do tipo $A_{2 k-1}$ (ver [24]). Já o conjunto $C(A)$ possui singularidades em $(0,0,0,[ \pm 1,1])$ e ambas são do tipo $A_{k-1}$.

\subsection{Desdobramento Versal}

Calculamos nessa seção alguns desdobramentos versais dos germes classificados no Capítulo 3 e estudamos com mais detalhes a geometria dos germes quando $r=n=2$. Referências para desdobramentos versais podem ser vistas em [22] ou [24].

Seja $A \in \mathcal{S}$ um germe $\mathcal{G}$-finitamente determinado. Pela Proposição 2.4.4 e Corolário 2.4.4 temos que $\mathcal{O}_{N} / T_{e} \mathcal{G} A$ é um $\mathbb{K}$-espaço vetorial finitamente gerado. Sejam $\phi_{1}, \ldots, \phi_{l} \in \mathcal{S}$ geradores deste espaço. Então $A+u_{1} \phi_{1}+\ldots+u_{l} \phi_{l}$ é um desdobramento versal do germe $A$.

Observamos que no próximo resultado as formas normais foram obtidas usando $\mathbb{K}=\mathbb{R}$.

Proposição 4.2.1. Os $\mathcal{G}$ desdobramentos versais de alguns germes simples do Capítulo 3 aparecem nos seguintes casos:

(1) Quando $r=1$, o desdobramento tem a forma $A=\left(A_{i j}(x, y, u)\right)$ onde $A_{11}=x^{m_{1}}+$ $\sum_{k=0}^{m_{1}-2} u_{1,1, k} x^{k}$ e $A_{i j}=A_{j i}=\delta_{i j} x^{m_{i}}+\sum_{k=0}^{m_{i}-1} u_{i j} x^{k}$ para $1 \leqslant i \leqslant j e 1<j \leqslant n$.

(2) Quando $n=1$ este coincide com desdobramento universal de germes de funções simples.

(3) Quando $r=n=2$ nós temos a seguinte tabela:

Forma Normal Desdobramento

1. $\left(x, y, \pm x^{k}\right)$

$\left(x, y, \pm x^{k}+\sum_{i=0}^{k-1} v_{i} x^{i}\right)$

2. $\left(x, y^{k}, x\right)$

$\left(x, y^{k}+\sum_{i=0}^{k-2} u_{i} y^{i}, x+\sum_{i=0}^{k-1} v_{i} y^{i}\right)$

3. $\left( \pm y^{k}, x, y^{l}\right)$

$\left( \pm y^{k}+\sum_{i=0}^{k-2} u_{i} y^{i}, x, y^{l}+\sum_{i=0}^{l-1} v_{i} y^{i}\right)$

4. $\left(x, 0, y^{2} \pm x^{k}\right)$

$\left(x, u_{1} y+u_{0}, y^{2} \pm x^{k}+\sum_{i=0}^{k-1} v_{i} x^{i}\right)$

5. $\left(x, 0, x y \pm y^{k}\right)$

$\left(x, \sum_{i=0}^{k-1} u_{i} y^{i}, x y \pm y^{k}+\sum_{i=0}^{k-1} v_{i} y^{i}\right)$

6. $\left(x, y^{k}, x y\right)$

$\left(x, y^{k}+\sum_{i=0}^{k-1} u_{i} y^{i}, x y+\sum_{i=0}^{k} v_{i} y^{i}\right)$

7. $\left(x, y^{2}, x^{2}\right)$

$\left(x, y^{2}+u_{0}, x^{2}+v_{4} y^{2}+v_{3} x y+v_{2} y+v_{1} x+v_{0}\right)$

8. $\left(x, 0, x^{2}+y^{3}\right)$

$\left(x, u_{2} y^{2}+u_{1} y+u_{0}, x^{2}+y^{3}+v_{3} x y+v_{2} y+v_{1} x+v_{0}\right)$ 
Demonstração. Consideramos o germe $A=\left(x, y, x^{k}\right)$. Seu $\mathcal{G}$-espaço tangente estendido é o $\mathcal{O}$-módulo gerado por $\left\{\left(1,0, k x^{k-1}\right),(0,1,0),(2 x, y, 0),\left(2 y, x^{k}, 0\right),(0, x, 2 y),\left(0, y, 2 x^{k}\right)\right\}$. Calculemos uma base para $\mathcal{O} / T_{e} \mathcal{G} . A$. Usando os elementos $(0,1,0),(2 x, y, 0),\left(2 y, x^{k}, 0\right),(0, x, 2 y)$, $\left(0, y, 2 x^{k}\right)$, podemos mostrar que todos os elementos de $\mathcal{O}$ estão em $T_{e} \mathcal{G}$. $A$, exceto $(1,0,0)$, $(0,0,1)$ e $\left(0,0, x^{l}\right)$, com $1 \leqslant l \leqslant k-1$. Porém, temos que $\left(1,0, k x^{k-1}\right) \in T_{e} \mathcal{G} . A$. Logo, uma base para $\mathcal{O} / T_{e} \mathcal{G} . A$ pode ser dada por $\left\{\left(0,0, x^{l}\right), 0 \leqslant l \leqslant k-1\right\}$ e o desdobramento versal do germe $A$ é dado por $\left(x, y, x^{k}+\sum_{i=0}^{k-1} v_{i} x^{i}\right)$. Os outros casos seguem de modo análogo.

\subsection{Geometria adicional $(\mathrm{r}=\mathrm{n}=2)$}

O trabalho de J.W.Bruce [5], teve como motivação original o estudo de equações diferenciais binárias que está diretamente relacionado ao estudo das matrizes simétricas com $r=n=2$. Descrevemos alguns resultados básicos em relação à topologia do discriminante e da variedade associada $M(A)$. Esses resultados valem em geral, ou seja, não estão restritos somente à germes simples. Começamos com um resultado geral sobre $M(A)$.

Proposição 4.3.1. Um elemento $A \in \mathcal{S}$ é $\mathcal{G}$-finitamente determinado se, e somente se, $M(A)$ é suave fora de $M(A)(0)$.

Observação 4.3.2. Pela proposição acima, se um germe $A \in \mathcal{S}$ é finitamente determinado então as singularidades de $M(A)$ podem ocorrer somente ao longo de $0 \times \mathbb{C} P^{n}$.

Abaixo, listamos os números e os tipos das singularidades de $M(A)$ e $D(A)$ para alguns germes simples.

Proposição 4.3.3. Os tipos de singularidades do discriminante e da variedade associada $M(A)$ dos germes simples obtidos no Teorema 3.3 .1 são:

$\begin{array}{lcc}\text { Forma normal } & \text { Discriminante } & \text { Pontos Singulares de } M(A) \\ \text { 1. }\left(y^{k}, x, y^{l}\right), k \geqslant 1, l \geqslant 2 & A_{k+l-1} & A_{k-1}+A_{l-1} \\ \text { 2. }\left(x, 0, y^{2}+x^{k}\right), k \geqslant 2 & D_{k+2} & D_{k+1} \\ \text { 3. }\left(x, 0, x y+y^{k}\right), k \geqslant 2 & D_{2 k} & A_{2 k-1} \\ \text { 4. }\left(x, y^{k}, x y\right), k \geqslant 2 & D_{2 k+1} & A_{2 k} \\ \text { 5. }\left(x, y^{2}, x^{2}\right) & E_{6} & D_{5} \\ \text { 6. }\left(x, 0, x^{2}+y^{3}\right) & E_{7} & E_{6}\end{array}$

Demonstração. 1. $\left(y^{k}, x, y^{l}\right)$. Temos $\delta=y^{k+l}-x^{2}=0$, que claramente tem uma singularidade $A_{k+l-1}$. Já $M(A)$ é dado por $y^{k} p^{2}+2 x p q+y^{l} q^{2}=0$. Tomando a carta afim $p=1$ em $\mathbb{C} P^{1}$, obtemos $y^{k}+2 x q+y^{l} q^{2}=0$, que tem uma singularidade $A_{k-1}$ em $(0,0,0)$. Assim $M(A)$ possui 
uma singularidade $A_{k-1}$ no ponto $(0,0,[1,0]) \in \mathbb{C}^{2} \times \mathbb{C} P^{1}$. Com a carta $q=1$, obtemos que $M(A)$ possui uma singularidade $A_{l-1}$ no ponto $(0,0,[0,1])$.

2. $\left(x, 0, y^{2}+x^{k}\right)$. O discriminante é dado por $\delta=x y^{2}+x^{k+1}=0$, que tem uma singularidade $D_{k+2}$. Já para $M(A)$, tomando a carta $p=1$, obtemos $x+y^{2} q^{2}+x^{k} q^{2}=0$, que é suave. Com a carta $q=1$, obtemos $x p^{2}+y^{2}+x^{k}=0$, que possui uma singularidade no ponto $(0,0,0)$ do tipo $D_{k+1}$. Portanto a única singularidade de $M(A)$ é no ponto $(0,0,[0,1]) \in \mathbb{C}^{2} \times \mathbb{C} P^{1}$, do tipo $D_{k+1}$.

3. $\left(x, 0, x y+y^{k}\right)$. O discriminante é dado por $\delta=y x^{2}+x y^{k}=0$, que tem uma singularidade $D_{2 k}$ (ver Exemplo 4.1.8(2)). Para $M(A)$, tomando a carta $p=1$, obtemos $x+x y q^{2}+y^{k} q^{2}=0$, que é suave. Para a carta $q=1$, obtemos $x p^{2}+x y+y^{k}$. Contas análogas ao Exemplo 4.1.8-(3), nos mostra que $M(A)$ possui uma singularidade $A_{2 k-1}$ em $(0,0,[0,1])$.

4. $\left(x, y^{k}, x y\right)$. O discriminante é dado por $\delta=y x^{2}-y^{2 k}=0$, que tem uma singularidade $D_{2 k+1}$. Para $M(A)$, tomando a carta $p=1$, obtemos $x+2 y^{k} q+x y q^{2}=0$, que é suave. Para a carta $q=1$, obtemos $x p^{2}+2 y^{k} p+x y$. Mudanças de coordenadas o reduzem a $x y+p^{2 k+1}$, que tem uma singularidade $A_{2 k}$.

5. $\left(x, y^{2}, x^{2}\right)$. O discriminante é dado por $\delta=x^{3}-y^{4}=0$, que tem uma singularidade $E_{6}$. Para $M(A)$, tomando a carta $p=1$, obtemos $x+2 y^{2} q+x^{2} q^{2}=0$, que é suave. Para a carta $q=1$, obtemos $x p^{2}+2 y^{2} p+x^{2}$. Mudanças de coordenadas o reduzem a $x^{2}+p^{4}+p y^{2}$, que tem uma singularidade $D_{5}$.

6. $\left(x, 0, x^{2}+y^{3}\right)$. Seu discriminante é dado por $\delta=x^{3}+x y^{3}=0$, que tem uma singularidade $E_{7}$. Para $M(A)$, tomando a carta $p=1$, obtemos $x+x^{2} q^{2}+y^{3} q^{2}=0$, que é suave. Para a carta $q=1$, obtemos $x p^{2}+x^{2}+y^{3}$. Mudanças de coordenadas o reduzem a $x^{2}+p^{4}+y^{3}$, que tem uma singularidade $E_{6}$.

Pela Proposição 4.0.7, se $A, B \in \mathcal{S}$ são $\mathcal{G}$-equivalentes então elas produzem um difeomorfismo entre as variedades associadas $M(A)$ e $M(B)$ ao longo de $0 \times \mathbb{C} P^{n-1}$. Quando $r=n=2$, $d A(0) \neq 0$ e $A$ é $\mathcal{G}$-finitamente determinado então $M(A)$ tem no máximo duas singularidades ao longo de $0 \times \mathbb{C} P^{n-1}$.

Temos os seguintes resultados:

Proposição 4.3.4. Sejam $F$ e $G$ dois desdobramentos $\mathcal{G}$-equivalentes de $A$. Então os desdobramentos das singularidades de $M(A)$ induzidos por $F$ e $G$ são $\mathcal{K}$-equivalentes.

Proposição 4.3.5. Suponha que posto $(d A(0)) \neq 0$ e seja $F$ um desdobramento versal de $A$. Então o desdobramento de $M(A)$ induzido por $F$ "desdobra" versalmente as singularidades de $M(A)$ (mesmo para o caso em que $M(A)$ possui dois pontos singulares).

Abaixo apresentamos alguns resultados sobre a variedade $M(A)$.

Proposição 4.3.6. (1) O discriminante é suave se, e somente se, A é $\mathcal{G}$-equivalente à $(1,0,1)$ ou $(1,0, x)$. 
(2) A variedade associada $M(A)$ é suave se, e somente se, o discriminante é suave ou possui uma singularidade de Morse $\left(A_{1}\right)$, no caso em que $A(0)=0$.

(3) Se posto $A(0)=1$ então o criminante e discriminante podem ser identificados e $M(A)$ é singular se, e somente se, $D(A)$ é singular. Neste caso $M(A)$ tem somente uma singularidade que é a suspensão da singularidade de $D(A)$.

Demonstração. Seja $A(x, y)=\left(\begin{array}{ll}a(x, y) & b(x, y) \\ b(x, y) & c(x, y)\end{array}\right)$. Provemos inicialmente o item (1). $(\Rightarrow)$ Considerando $\delta(x, y)=a(x, y) \cdot c(x, y)-b^{2}(x, y)$, segue que

$$
\begin{gathered}
\delta_{x}=a_{x} \cdot c+a \cdot c_{x}-2 b \cdot b_{x}, \mathrm{e} \\
\delta_{y}=a_{y} \cdot c+a \cdot c_{y}-2 b \cdot b_{y} .
\end{gathered}
$$

Por hipótese temos que $D(A)$ é suave, ou seja, que ao menos uma das derivadas de $\delta$ não se anula em $(0,0)$, o que implica que pelo menos uma das funções $a, b, c$ não se anula em $(0,0)$. Logo, a matriz $A(0)$ tem posto 1 ou 2. Pela Proposição 2.5.3, temos que $A$ é $\mathcal{G}$-equivalente a $(1,0,1)$ ou $(1,0, f)$, mas no último caso, $f$ deve ser uma submersão e assim podemos reduzi-lo à $(1,0, x)$.

$(\Leftarrow)$ Trivial.

Para (2), ver [6], por exemplo.

(3) Pela Proposição 2.5.3, se posto $A(0)=1$ então podemos reduzir $A$ ao germe $(1,0, f(x, y))$, onde $f(0,0)=0$. Logo,

$$
\begin{gathered}
D(A)=\left\{(x, y) \in \mathbb{C}^{2} ; f(x, y)=0\right\} \\
M(A)=\left\{(x, y,[p, q]) \in \mathbb{C}^{2} \times \mathbb{C}^{1} ; p^{2}+q^{2} f(x, y)=0\right\} \text { e } \\
C(A)=\left\{(x, y,[p, q]) \in \mathbb{C}^{2} \times \mathbb{C}^{1} ; p+q f(x, y)=0 \text { e } f(x, y)=0\right\} .
\end{gathered}
$$

Observe que $D(A)$ é singular se, e somente se, $f$ é singular em $(0,0)$. Vejamos as possíveis singularidades em $M(A)$. Parametrizando $\mathbb{C} P^{1}$ com a carta $p=1$, obtemos $1+f(x, y) q^{2}=0$, que é suave. Por outro lado, com $q=1$ obtemos $p^{2}+f(x, y)=0$, que tem uma única singularidade na fibra $(0,0,[0,1])$ se, e somente se, $(0,0)$ é uma singularidade de $f$. Portanto, $M(A)$ é singular se, e somente se, $D(A)$ é singular e a singularidade de $M(A)$ é uma suspensão da singularidade de $f$, ou seja, da singularidade de $D(A)$.

Quanto a afirmação que $C(A)$ e $D(A)$ podem ser identificados, segue do fato que $C(A)=$ $D(A) \times[0,1]$.

Observamos que também para a variedade associada temos um resultado que relaciona o número de Milnor do discriminante $D(A)$ e das singularidades da variedade $M(A)$.

Teorema 4.3.7 ([16]). Se $A:\left(\mathbb{C}^{2}, 0\right) \rightarrow \operatorname{Sim}_{2}$ tem posto $(d A(0)) \neq 0$ e $\mathcal{G}$-codimensão finita então $M(A)$ tem 1 ou 2 pontos singulares e a soma de seus números de Milnor $\mu(M(A))$ é igual a $\mu(D(A))-1$. 


\section{Apêndice A}

\section{Caracterização geométrica}

Vimos na Proposição 2.4.6 que assim como para os grupos de Mather também temos uma caracterização geométrica para os germes $\mathcal{G}$-finitamente determinados. O objetivo deste apêndice é exibir a prova deste resultado para o grupo $\mathcal{G}$ seguindo os argumentos de James Damon para o grupo $\mathcal{K}_{V}([10])$. A prova de James Damon segue praticamente os mesmos argumentos de Mather para a caracterização geométrica do grupo $\mathcal{K}$. Também exibimos nesse apêndice alguns resultados de determinação finita para o grupo $\mathcal{G}$ que não foram enunciados na seção 2.4 .

Vamos inicialmente introduzir uma linguagem adaptada ao estudo das propriedades locais dos conjuntos. Uma referência é [23].

\section{Germes de conjuntos}

Definimos uma relação de equivalência em subconjuntos de $\mathbb{C}^{n}$ da seguinte maneira:

Definição A.0.8. Dizemos que dois subconjuntos $X, Y \subset \mathbb{C}^{n}$ são equivalentes em um ponto $a \in \mathbb{C}^{n}$ se existir uma vizinhança $U$ de a tal que $X \cap U=Y \cap U$. A classe de equivalência do conjunto $X$ em a é chamada de germe do conjunto $X$ em a e é denotada por $(X, a)$. Quando o ponto a estiver subentendido, denotamos apenas por $X$.

De maneira evidente definimos a relação de inclusão entre germes e as operações de união e intersecção. Por exemplo, dizemos que $X \supset Y(X, Y$ germes de conjunto em $a)$ se $X$ e $Y$ podem ser representados por conjuntos $S, T$ respectivamente tais que $S \supset T$.

Definição A.0.9. Sejam I um ideal de $\mathcal{O}_{n}$ gerado por $\gamma_{1}, \ldots, \gamma_{r}$ e $U$ uma vizinhança de 0 tal que $\gamma_{i}$ possua um representante $f_{i}$ em $U, 1 \leqslant i \leqslant r$. Definimos o germe $V(I)$ dos zeros de $I$ como sendo o germe do conjunto $\left\{x \in \mathbb{C}^{n} ; f_{1}(x)=\ldots=f_{r}(x)=0\right\}$.

Seja $f \in \mathcal{O}_{n}$ e $X$ um germe de um conjunto em 0 . Dizemos que $f$ se anula sobre $X$ quando $X$ pode ser representado por um conjunto $S$ tal que $f_{\mid S}=0$. 
Definição A.0.10. Seja $X$ um germe de um conjunto em $0 \in \mathbb{C}^{n}$. Definimos o conjunto

$$
\mathcal{I}(X)=\left\{f \in \mathcal{O}_{n} ; f \text { se anula sobre } X\right\}
$$

que é um ideal de $\mathcal{O}_{n}$ chamado de ideal de $X$.

Proposição A.0.11. Sejam $X, Y$ germes de conjuntos em $0 \in \mathbb{C}^{n}$ e $I, J$ ideais em $\mathcal{O}_{n}$. Temos as seguintes propriedades:
a) $\quad S e X \subset$ então $\mathcal{I}(X) \supset \mathcal{I}(Y)$
b) $\mathcal{I}(X \cup Y)=\mathcal{I}(X) \cap \mathcal{I}(Y)$
c) $S e I \subset J$ então $V(I) \supset V(J)$
d) $V(I+J)=V(I) \cap V(J)$
e) $V(I \cap J)=V(I . J)=V(I) \cup V(J)$

Demonstração. Ver ([23], p. 69).

Dado um anel $I$ de $\mathcal{O}_{n}$ definimos o ideal $\operatorname{Rad}(I)=\left\{f \in \mathcal{O}_{n} ; \exists m \in \mathbb{N}\right.$ tal que $\left.f^{m} \in I\right\}$, também denotado por $\sqrt{I}$.

Teorema A.0.12 (Teorema de Hilbert Nullstellensatz). Seja I um ideal de $\mathcal{O}_{n}$. Então $\mathcal{I}(V(I))=\operatorname{Rad}(I)$.

Demonstração. Ver ([23], p. 73).

\section{Caracterização geométrica dos germes finitamente determinados}

Iniciemos com algumas definições. Dado um germe $A:\left(\mathbb{K}^{r}, 0\right) \rightarrow \operatorname{Sim}_{n}(\mathbb{K})$ definimos a seguinte função associada a este germe:

$$
\begin{array}{ccc}
\delta_{A}:\left(\mathbb{K}^{r}, 0\right) & \longrightarrow & \mathbb{K} \\
x & \longmapsto \operatorname{det}(A(x)) .
\end{array}
$$

Definição A.0.13. Denotamos por $I_{A}$ o ideal em $\mathcal{O}_{r}$ gerado pela função $\delta_{A}$.

Exemplo A.0.14. Considere $A:\left(\mathbb{C}^{2}, 0\right) \rightarrow \operatorname{Sim}_{2}(\mathbb{C})$, dada por $A(x, y)=\left(\begin{array}{cc}x & y^{2} \\ y^{2} & x y\end{array}\right)$. Então $\delta_{A}:\left(\mathbb{C}^{2}, 0\right) \rightarrow \mathbb{C}$ será dada por $\delta_{A}(x, y)=x^{2} y-y^{4}$ e $I_{A}$ será o ideal em $\mathcal{O}_{2}$ gerado por $x^{2} y-y^{4}$.

Dado um germe $A:\left(\mathbb{K}^{r}, 0\right) \rightarrow \operatorname{Sim}_{n}(\mathbb{K})$, denotaremos por $T A$ a matriz $N \times P$, onde $N=\frac{n(n+1)}{2}$ e $P=r+n^{2}$, cujas colunas são as coordenadas de $A_{x_{1}}, \ldots, A_{x_{r}}, A_{i j}$ em relação à base canônica de $\operatorname{Sim}_{n}$.

Definição A.0.15. Denotamos por $J_{A}$ o ideal em $\mathcal{O}_{r}$ gerado por todos os determinantes das submatrizes $N \times N$ de $T A$. 
Exemplo A.0.16. Considere $A:\left(\mathbb{C}^{2}, 0\right) \rightarrow \operatorname{Sim}_{2}(\mathbb{C})$, dada por $A(x, y)=\left(\begin{array}{cc}x & y^{3} \\ y^{3} & x y\end{array}\right)$. Temos $A_{x}=\left(\begin{array}{ll}1 & 0 \\ 0 & y\end{array}\right), A_{y}=\left(\begin{array}{cc}0 & 3 y^{2} \\ 3 y^{2} & x\end{array}\right), A_{11}=\left(\begin{array}{cc}2 x & y^{3} \\ y^{3} & 0\end{array}\right), A_{12}=\left(\begin{array}{cc}2 y^{3} & x y \\ x y & 0\end{array}\right)$, $A_{21}=\left(\begin{array}{cc}0 & x \\ x & 2 y^{3}\end{array}\right), A_{22}=\left(\begin{array}{cc}0 & y^{3} \\ y^{3} & 2 x y\end{array}\right)$. Logo,

$$
T A=\left(\begin{array}{cccccc}
1 & 0 & 2 x & 2 y^{3} & 0 & 0 \\
0 & 3 y^{2} & y^{3} & x y & x & y^{3} \\
y & x & 0 & 0 & 2 y^{3} & 2 x y
\end{array}\right) .
$$

Todos os subdeterminantes $3 \times 3$ dessa matriz são os geradores de $J_{A}$.

Observação A.0.17. Seja $A:\left(\mathbb{K}^{r}, 0\right) \rightarrow \operatorname{Sim}_{n}(\mathbb{K})$. Dado $B \in T_{e} \mathcal{G} . A$, existem $f_{1}, \ldots, f_{P} \in$ $\mathcal{O}_{r}$ tais que

$$
f_{1} A_{x_{1}}+\ldots+f_{r} A_{x_{r}}+f_{r+1} A_{11}+\ldots+f_{P} A_{n n}=B .
$$

Considerando o "vetor" $v=\left(f_{1}, \ldots, f_{P}\right)$, segue que $T A(v)$ será um germe $\mathbb{K}^{r} \rightarrow \mathbb{K}^{N}$ que ao identificarmos $\mathbb{K}^{N}$ com $\operatorname{Sim}_{n}(\mathbb{K})$ obtemos $B$.

Exemplo A.0.18. Considere A como no Exemplo A.0.16. Tomando $f_{1}(x, y)=x, f_{3}(x, y)=$ $x y, f_{6}(x, y)=x y^{2}$ e as outras identicamente nulas, temos $v=\left(f_{1}, 0, f_{3}, 0,0, f_{6}\right) e$

$$
T A(v)=T A=\left(\begin{array}{cccccc}
1 & 0 & 2 x & 2 y^{3} & 0 & 0 \\
0 & 3 y^{2} & y^{3} & x y & x & y^{3} \\
y & x & 0 & 0 & 2 y^{3} & 2 x y
\end{array}\right)\left(\begin{array}{c}
f_{1} \\
0 \\
f_{3} \\
0 \\
0 \\
f_{6}
\end{array}\right)=\left(\begin{array}{c}
x+2 x^{2} y \\
x y^{4}+x y^{5} \\
x y+2 x^{2} y^{3}
\end{array}\right) .
$$

Identificando o germe $B:\left(\mathbb{C}^{2}, 0\right) \rightarrow \mathbb{C}^{3}$ com o germe

$$
\begin{aligned}
\widetilde{B}:\left(\mathbb{C}^{2}, 0\right) & \longrightarrow \\
(x, y) & \longmapsto\left(\begin{array}{cc}
x+2 x^{2} y & x y^{4}+x y^{5} \\
x y^{4}+x y^{5} & x y+2 x^{2} y^{3}
\end{array}\right),
\end{aligned}
$$

claramente vemos que $\widetilde{B}=f_{1} A_{x}+f_{3} A_{11}+f_{6} A_{22}$.

Durante o texto, quando nos referirmos a $T A$ sempre estaremos pensando nessas identificações.

Lema A.0.19. Para todo germe $A \in \mathcal{S}$ temos $I_{A} \mathcal{S} \subset T \mathcal{G} . A$ 
Demonstração. Dado um par $(i, j), 1 \leqslant i \leqslant j \leqslant n$, queremos mostrar que $\delta_{A} \cdot E_{i j} \in T \mathcal{G} . A$. Tomando $B=\sum_{k=1}^{n} \widehat{M_{j k}} A_{i k} \in T \mathcal{G} . A$, onde $\widehat{M_{j k}}$ é o cofator do elemento da $(j, k)$ entrada de $A$. Afirmamos que $B=\delta_{A} \cdot E_{i j}$.

De fato, primeiro notemos que em uma posição que esteja fora da $i$-ésima linha ou $i$-ésima coluna de $B$ não aparece soma alguma, logo será identicamente nulo. Em uma entrada de $B$ que esteja na $i$-ésima linha, mas não na $j$-ésima coluna será identicamente nulo, pois será o determinante de uma matriz com colunas repetidas. O mesmo argumento para uma entrada de $B$ que esteja na $i$-ésima coluna e que não esteja na $j$-ésima linha. Nas $(i, j)$ e $(j, i)$ entradas de $B$ temos $\delta_{A}$ (basta desenvolvermos o determinante de $A$ pela sua $j$-ésima coluna).

Lema A.0.20. Sejam $I$ e $H$ ideais em $\mathcal{O}_{r}$ tais que $T A\left(\mathcal{O}^{P}\right)+I \mathcal{S} \supset H \mathcal{S}$, então $J_{A}+I \supset H^{N}$ Demonstração. Segue análogo a ([26], p. 512).

Proposição A.0.21. Um elemento $A \in \mathcal{S}$ é $\mathcal{G}$-finitamente determinado se, e somente se, $J_{A}+I_{A} \supset \mathcal{M}_{r}^{l}$, para algum $l \in \mathbb{N}$.

Demonstração. Suponhamos $A$ finitamente determinado. Pelo Corolário 2.4.4 que existe $k \in$ $\mathbb{N}$ tal que $\mathcal{O}\left\{A_{x_{i}}, A_{i j}\right\} \supset \mathcal{M}^{k} \mathcal{S}$. Pelo Lema A.0.19, temos $I_{A} \mathcal{S} \subset T \mathcal{G} . A$ e assim $\mathcal{O}\left\{A_{x_{i}}, A_{i j}\right\}+$ $I_{A} \mathcal{S} \supset \mathcal{M}_{r}^{k} \mathcal{S}$. Aplicando o lema acima para $I=I_{A}$ e $H=\mathcal{M}_{r}^{k}$, segue que $J_{A}+I_{A} \supset \mathcal{M}_{r}^{k N}$.

Por outro lado, suponhamos $J_{A}+I_{A} \supset \mathcal{M}_{r}^{k}$. É suficiente mostrarmos que $J_{A} \mathcal{S} \subset T_{e} \mathcal{G} . A$, pois $I_{A} \mathcal{S} \subset T_{e} \mathcal{G} . A$ pelo Lema A.0.19. Denote por $T A_{\left(i_{1}, \ldots, i_{N}\right)}$ a matriz $N \times N$ cuja $j$-ésima coluna é a $i_{j}$-coluna de $T A, 1 \leqslant j \leqslant P$.

Os elementos da forma $\delta_{\left(T A_{\left(i_{1}, \ldots, i_{N}\right)}\right)} \cdot E_{R} \in \mathcal{S}$ geram $J_{A} \mathcal{S}$, onde $E_{R}$ são os vetores da base canônica de $\operatorname{Sim}_{n}(\mathbb{C})$ e $1 \leqslant R \leqslant N$. Considerando $\widehat{M}_{i_{s}}^{r}$ o cofator do elemento que está na $R$-ésima linha e $i_{s}$-ésima coluna de $T A_{\left(i_{1}, \ldots, i_{N}\right)}$, afirmamos que

$$
\delta_{\left(T A_{\left(i_{1}, \ldots, i_{N}\right)}\right)} E_{R}=T A\left(\sum_{s=1}^{N} \widehat{M}_{i_{s}}^{r} \epsilon_{i_{s}}\right) .
$$

De fato, denotando por $\alpha_{i j}$ o elemento da $(i, j)$ posição em $T A$, temos na $R$-ésima posição do lado direito da igualdade acima o elemento

$$
\sum_{s=1}^{N} \alpha_{R i_{s}} \widehat{M}_{i_{s}}^{r}=\delta_{\left(T A_{\left(i_{1}, \ldots, i_{N}\right)}\right)}
$$

Quando $1 \leqslant J \leqslant N$ e $J \neq R$ teremos na $J$-ésima posição

$$
\sum_{s=1}^{N} \alpha_{J i_{s}} \widehat{M}_{i_{s}}^{r}
$$

mas esse elemento será sempre igual a zero, pois é o determinante de uma matriz com colunas repetidas. 
Proposição A.0.22. Um germe $A:\left(\mathbb{C}^{r}, 0\right) \rightarrow \operatorname{Sim}_{n}(\mathbb{C})$ é $\mathcal{G}$-finitamente determinado se, e somente se, A é transversal à estratificação de $\operatorname{Sim}_{n}(\mathbb{C})$ fora da origem.

Demonstração. Pela Observação 2.4 .5 basta nos preocuparmos com os elementos $z_{0} \in \mathbb{C}^{r}$ em que $A\left(z_{0}\right)$ não é invertível. Pela Proposição A.0.21 um elemento $A \in \mathcal{S}$ é $\mathcal{G}$-finitamente determinado $\Leftrightarrow J_{A}+I_{A} \supset \mathcal{M}_{r}^{k}$, para algum $k \in \mathbb{N}$.

Suponhamos que $A$ é $\mathcal{G}$-finitamente determinado. Pelo Teorema A.0.12 temos

$$
\sqrt{J_{A}+I_{A}}=\mathcal{I}\left(V\left(J_{A}+I_{A}\right)\right)=\mathcal{I}\left(V\left(J_{A}\right) \cap V\left(I_{A}\right)\right) .
$$

Logo, $\mathcal{I}\left(V\left(J_{A}\right) \cap V\left(I_{A}\right) \supset \mathcal{M}_{r}\right.$. Como $\mathcal{M}_{r}$ é maximal segue que $\mathcal{I}\left(V\left(J_{A}\right) \cap V\left(I_{A}\right)\right)=\mathcal{O}$ ou $\mathcal{I}\left(V\left(J_{A}\right) \cap V\left(I_{A}\right)\right)=\mathcal{M}_{r}$. Como a primeira igualdade não pode ocorrer temos $\mathcal{I}\left(V\left(J_{A}\right) \cap\right.$ $\left.V\left(I_{A}\right)\right)=\mathcal{M}_{r}$, ou seja,

$$
V\left(J_{A}\right) \cap V\left(I_{A}\right)=\{0\} .
$$

Mas, $V\left(J_{A}\right) \cap V\left(I_{A}\right)$ é a interseção dos pontos $z_{0} \in \mathbb{C}^{r}$ em que a matriz $A\left(z_{0}\right)$ não é invertível com o conjunto dos pontos $z_{0} \in \mathbb{C}^{r}$ em que $A$ não é transversal à estratificação de $\operatorname{Sim}_{n}(\mathbb{C})$. Portanto, em qualquer ponto fora da origem temos que $A$ é transversal à estratificação de $\operatorname{Sim}_{n}(\mathbb{C})$.

Por outro lado, suponhamos que $A$ é transversal à estratificação de $\operatorname{Sim}_{n}(\mathbb{C})$ fora da origem. Segue que $V\left(J_{A}\right) \cap V\left(I_{A}\right)=\{0\} \Rightarrow \mathcal{I}\left(V\left(J_{A}\right) \cap V\left(I_{A}\right)\right)=\mathcal{M}_{r}$. Pelo Nullstelensatz temos $\mathcal{I}\left(V\left(J_{A}\right) \cap V\left(I_{A}\right)\right)=\sqrt{J_{A}+I_{A}}$, ou seja,

$$
\sqrt{J_{A}+I_{A}}=\mathcal{M}_{r} \Rightarrow J_{A}+I_{A} \supset \mathcal{M}_{r}^{k}, \text { para algum } k \in \mathbb{N}
$$

Portanto $A$ é finitamente determinado. 


\section{Apêndice B}

\section{Símbolo Segre}

Na seção 2.5 fornecemos um resultado sobre pencils de cônicas. Neste apêndice vamos descrever um pouco sobre esta classificação e alguns conceitos relacionados a ela, em especial para entendermos o Símbolo Segre.

Dado um par de cônicas $f, g$ podemos associá-las às matrizes $F, G \in \operatorname{Sim}_{3}(\mathbb{C})$, respectivamente. Esse par de matrizes determina um pencil de matrizes $\mathcal{P}=\left\{\lambda F+\mu G /(\lambda, \mu) \in \mathbb{P}^{1}\right\}$. Pencils de cônicas são classificadas de acordo com o seu Símbolo Segre. O Símbolo Segre de um pencil de quadrática complexa $X$ é por definição o Símbolo Segre do associado pencil de matrizes.

Definição B.0.23. O discriminante de um pencil $\mathcal{P}$ é a cúbica binária

$$
\Delta=\Delta_{(\lambda, \mu)}:=\operatorname{det}(\lambda F+\mu G)
$$

Quando $\Delta$ não se anula identicamente, dizemos que o pencil é não singular, e do contrário dizemos que ele é singular. Suponha que $\mathcal{P}$ é não singular e que $(\bar{\lambda}, \bar{\mu})$ é uma raiz de $\Delta$. Pode acontecer que todos os subdeterminantes de uma ordem menor de $\bar{\lambda} F+\bar{\mu} G$ se anulem, sendo assim, suponha que todos os subdeterminantes de ordem $3-d$ se anulem e que isto não aconteça para todos os subdeterminantes de ordem $2-d$.

Denotaremos $l_{i}$ como sendo a menor multiplicidade da raiz $(\bar{\lambda}, \bar{\mu})$ nos subdeterminantes de ordem $3-i$, para $i=0,1, . ., d, \operatorname{logo} l_{i}>l_{i+1}$ para todo $i$ e assim $e_{i}:=l_{i}-l_{i+1}>0 . \operatorname{Logo}$,

$$
\Delta(\lambda, \mu)=(\lambda \bar{\mu}-\bar{\lambda} \mu)^{e_{0}} \ldots(\lambda \bar{\mu}-\bar{\lambda} \mu)^{e_{d}} \Delta_{1}(\lambda, \mu),
$$

com $\Delta_{1}(\bar{\lambda}, \bar{\mu}) \neq 0$. Os números $e_{i}$ são chamados de números característicos da raiz $(\bar{\lambda}, \bar{\mu}) \mathrm{e}$ os fatores $(\lambda \bar{\mu}-\bar{\lambda} \mu)^{e_{i}}$ são chamados de divisores elementares do pencil $\mathcal{P}$.

Definição B.0.24 (Símbolo Segre). Se $\left(\lambda_{j}, \mu_{j}\right)$ para $j=1,2, \ldots, r$, são as raízes de $\Delta e$ $e_{0}^{j}, \ldots, e_{d_{j}}^{j}$ os números característicos associados à raiz $\left(\lambda_{j}, \mu_{j}\right)$ e $d_{1} \geqslant d_{2}, \ldots, \geqslant d_{r}$, então 


$$
\sigma_{\mathcal{P}}=\left[\left(e_{0}^{1}, \ldots, e_{d_{1}}^{1}\right),\left(e_{0}^{2}, \ldots, e_{d_{2}}^{2}\right), \ldots\left(e_{0}^{r}, \ldots, e_{d_{r}}^{r}\right)\right]
$$

é chamado o Símbolo Segre da quadrática complexa $X$ ou do pencil $\mathcal{P}$.

Em [17], mostra-se que dois pencils $\mathcal{P}_{1}$ e $\mathcal{P}_{2}$ de cônicas são equivalentes se, e somente se, elas possuem o mesmo Símbolo Segre. Podemos usar isto para exibirmos formas normais para pencils, cujo discriminante não se anula identicamente. Ver [4] ou [17].

Para cada $e_{j}^{i}$ ocorrendo no Símbolo Segre de $X$, considere as matrizes de ordem $e_{j}^{i} \times e_{j}^{i}$ :

$$
F_{i j}=\left(\begin{array}{cccc}
0 & \ldots & 1 & \frac{\lambda_{i}}{\mu_{j}} \\
\vdots & \ldots & \ldots & 0 \\
1 & \frac{\lambda_{i}}{\mu_{j}} & 0 & \vdots \\
\frac{\lambda_{i}}{\mu_{j}} & \ldots & \ldots & 0
\end{array}\right) \quad \text { e } \quad G_{i j}=\left(\begin{array}{cccc}
0 & \ldots & \ldots & 1 \\
\vdots & \ldots & 1 & 0 \\
\vdots & \ldots & \ldots & \vdots \\
1 & 0 & \ldots & 0
\end{array}\right)
$$

Assim $F$ e $G$, são formadas por blocos dessas matrizes da seguinte forma

$$
F=\operatorname{diag}\left(F_{11}, \cdots, F_{r d_{r}}\right) \text { e } G=\operatorname{diag}\left(G_{11}, \cdots, G_{r d_{r}}\right)
$$

Para o caso em que discriminante se anula identicamente, temos um isomorfismo em soma direta $\rho \oplus \sigma_{i_{1}} \oplus \ldots \oplus \sigma_{i_{k}}$, onde $\rho$ representa um pencil não singular e $\sigma_{i}$ tem a forma:

$$
\sigma_{i}\left(x_{1}, \ldots, x_{2 i-1}\right)=\left(x_{1} x_{2}+\ldots+x_{2 i-3} x_{2 i-2}, x_{2} x_{3}+\ldots+x_{2 i-2} x_{2 i-1}\right)
$$

Nessas condições após o símbolo de $\rho$ adicionaremos ponto-vírgula e os números $i_{1}, \ldots i_{k}$.

Exemplo B.0.25. Considere o Símbolo Segre $\{1,1,1\}$, ele representa um pencil que possui três raizes distintas em $\mathbb{C} P^{1}$. Tomaremos para esse pencil as raízes $\left(\lambda_{1}, \mu_{1}\right)=[0,1] ;\left(\lambda_{2}, \mu_{2}\right)=$ $[1,1] ;\left(\lambda_{3}, \mu_{3}\right)=[1,-1]$. Segue, que:

$$
F_{11}=(0) \text { e } G_{11}=(1) ; F_{22}=(1) \text { e } G_{22}=(1) ; F_{33}=(-1) \text { e } G_{33}=(1)
$$

e como acima, temos:

$$
F=\operatorname{diag}\left(F_{11}, F_{22}, F_{33}\right)=\left(\begin{array}{ccc}
0 & 0 & 0 \\
0 & 1 & 0 \\
0 & 0 & -1
\end{array}\right) ; G=\operatorname{diag}\left(G_{11}, G_{22}, G_{33}\right)=\left(\begin{array}{ccc}
1 & 0 & 0 \\
0 & 1 & 0 \\
0 & 0 & 1
\end{array}\right)
$$

Logo, o pencil $\mathcal{P}$ é dada por $\lambda F+\mu G$. Tomando as quadráticas associadas a $F$ e $G$, obtemos $\left(-y^{2}+z^{2}, x^{2}+y^{2}+z^{2}\right)$. Usando o difeomorfismo $\phi(x, y, z)=(z, y-x, y+x)$, temos que $\left(-y^{2}+z^{2}, x^{2}+y^{2}+z^{2}\right)$ será equivalente a $\left(4 x y, 2 x^{2}+2 y^{2}+z^{2}\right)$, e por mudanças escalares e nos geradores do pencil podemos reduzir a $\left(x y, x^{2}+y^{2}+z^{2}\right)$, conforme Proposição 2.6.3. 
Exemplo B.0.26. Considere $\left(x y, y^{2}+z^{2}\right)$, tomando as matrizes correspondentes a esse par de quadráticas, obtemos:

$$
F=\left(\begin{array}{lll}
0 & 1 & 0 \\
1 & 0 & 0 \\
0 & 0 & 0
\end{array}\right) \quad e G=\left(\begin{array}{lll}
0 & 0 & 0 \\
0 & 1 & 0 \\
0 & 0 & 1
\end{array}\right)
$$

Considerando o pencil $\mathcal{P}=\{\lambda F+\mu G / \lambda, \mu \in \mathbb{C}\}$, temos que seu discriminante será dado por $\Delta(\lambda, \mu)=\lambda^{2} \mu$. Suas raízes em $\mathbb{C} P^{1}$ são $(0,1)$, com multiplicidade 2 , e $(1,0)$ com multiplicidade 1. Podemos ver que ambas não anulam todos subdeterminantes de ordem 2, então $d=0, e_{1}=2$ e $e_{2}=1$. Portanto, seu Símbolo Segre será $\{2,1\}$.

No caso de cônicas temos 12 formas normais e elas podem ser vistas em ([27], p. 477). 


\section{Apêndice C}

\section{$\mathcal{K}_{V}$-equivalência}

Damon, em [11] e [10], apresenta uma relação de equivalência para seções de uma variedade $V$ através de um subgrupo do grupo de contato $\mathcal{K}$. Em nosso contexto, $V$ será o conjunto das matrizes simétricas com determinante nulo. Passaremos a apresentar algumas definições e resultados desta teoria. Iniciamos com algumas definições básicas.

\section{Campo de vetores}

Seja $M^{m} \subset \mathbb{K}^{n}$ uma variedade diferenciável suave de dimensão $m$. Um campo de vetores em $M$ é uma aplicação $\nu: M \rightarrow \mathbb{K}^{n}$. O campo $\nu$ diz-se tangente à variedade $M$ quando $\nu(p) \in T_{p} M$ para todo $p \in M$. Um subconjunto aberto é ainda uma variedade suave. Logo tem sentido considerar campos de vetores tangentes definidos em $U$.

Dada uma parametrização $\varphi: U \subset \mathbb{K}^{m} \rightarrow U_{0} \subset M$, os vetores $\frac{\partial \varphi}{\partial x_{1}}(x), \ldots, \frac{\partial \varphi}{\partial x_{m}}(x)$, constituem, para cada $x \in U$, uma base de $T_{p} M$, onde $\varphi(x)=p$.

Seja $\nu: M \rightarrow \mathbb{K}^{n}$ um campo de vetores tangentes. Em cada ponto $p=\varphi(x)$ da vizinhança parametrizada $U_{0}$ o vetor $\nu(p)$ se escreve como combinação linear dos vetores básicos $\frac{\partial \varphi}{\partial x_{j}}(x) \in$ $T_{p} M$, assim:

$$
\nu(p)=\sum_{j=1}^{m} \alpha_{j}(x) \frac{\partial \varphi}{\partial x_{j}}(x), p=\varphi(x) .
$$

Isto define $m$ funções $\alpha_{1}, \ldots, \alpha_{m}: U \rightarrow \mathbb{K}$. Em [20], mostra-se que $\nu \in C^{r}$ se, e somente se, as funções $\alpha_{1}, \ldots, \alpha_{m}: U \rightarrow \mathbb{K}$ acima definidas são de classe $C^{r}$.

Estaremos considerando germes de aplicações $\left(\mathbb{K}^{r}, 0\right)$ em $\operatorname{Sim}_{n}(\mathbb{K})$, ou seja, aplicações entre espaços vetoriais. $\operatorname{Logo}, M=\mathbb{K}^{t}$ para algum $t$, e neste caso um campo de vetores tangente nada mais será do que uma aplicação $\nu: \mathbb{K}^{t} \rightarrow \mathbb{K}^{t}$. Sempre tomaremos $\varphi=i d_{t} \mathrm{e}$ 
assim

$$
\nu(p)=\sum_{j=1}^{t} \alpha_{j}(p) \frac{\partial}{\partial x_{j}}
$$

onde $\frac{\partial}{\partial x_{j}}=\frac{\partial i d_{t}}{\partial x_{j}}(p)=\epsilon_{j}$ e $\epsilon_{j}$ é o $j$-ésimo vetor da base canônica de $\mathbb{K}^{t}$.

Denotando por $\mathcal{E}$ o conjunto das funções em $\mathbb{K}^{n}, f: \mathbb{K}^{n} \rightarrow \mathbb{K}$, será conveniente pensarmos em um campo vetorial $\nu$ como uma aplicação $\nu: \mathcal{E} \rightarrow \mathcal{E}$, dada por:

$$
\nu(f)(p)=\sum_{j=1}^{n} \alpha_{j}(p) \frac{\partial f}{\partial x_{j}}(p) .
$$

Definição C.0.27 (Campo de Euler). Dizemos que um campo de vetores tangentes $\nu$ em $\mathbb{K}^{n}$ é um campo de Euler se existem inteiros $a_{1}, \ldots, a_{n}$ tais que

$$
\nu=\sum_{j=1}^{n} a_{j} x_{j} \frac{\partial}{\partial x_{j}}
$$

\section{$\mathcal{K}_{V}$-equivalência.}

Definição C.0.28. Seja $(V, 0) \subset \mathbb{C}^{t}, 0$ um germe de uma variedade. Denotaremos por $\mathcal{K}_{V}$ o seguinte subgrupo de $\mathcal{K}$ :

$$
\mathcal{K}_{V}=\left\{H \in \mathcal{K}: H\left(\mathbb{C}^{s} \times V\right) \subseteq \mathbb{C}^{s} \times V\right\}
$$

Em particular, se $V=\{0\}$ então este é o grupo $\mathcal{K}$.

Claramente $\mathcal{K}_{V}$ é um subgrupo de $\mathcal{K}$ e sua ação nos germes de aplicações é definida da mesma forma que a ação de $\mathcal{K}$. Em [10], mostra-se que $\mathcal{K}_{V}$ é um subgrupo geométrico de Damon e como consequência dos resultados de Damon pode-se usar todas as técnicas da teoria de singularidades.

Considere o germe de uma variedade $(V, 0)$ e $I(V)$ o ideal dos germes que se anulam em $V$. Definimos por Derlog(V) o conjunto:

$$
\Theta_{V}=\left\{\zeta \in \theta_{t}: \zeta(I(V)) \subseteq I(V)\right\}
$$

Exemplo C.0.29. Seja $(V, 0) \subset\left(\mathbb{C}^{4}, 0\right)$ definida por y $w^{2}-z^{2}=0$. Claramente $I(V)$ é o ideal gerado por $g(x, y, z, w)=y w^{2}-z^{2}$ e os elementos de $\theta_{4}$ abaixo, pertencem à Derlog $(V)$.

$$
2 y \frac{\partial}{\partial y}+z \frac{\partial}{\partial z}, \quad z \frac{\partial}{\partial z}+w \frac{\partial}{\partial w}, \quad w y \frac{\partial}{\partial z}+z \frac{\partial}{\partial w}, \quad 2 z \frac{\partial}{\partial y}+w^{2} \frac{\partial}{\partial z}, \quad \frac{\partial}{\partial x}
$$

De fato, tomando por exemplo o campo $2 y \frac{\partial}{\partial y}+z \frac{\partial}{\partial z}$, temos:

$$
2 y \frac{\partial g}{\partial y}+z \frac{\partial g}{\partial z}=2 y w^{2}+\left(-2 z^{2}\right)=2\left(y w^{2}-z^{2}\right) \in I(V) .
$$


Obtemos abaixo quem é o espaço tangente à órbita de um dado germe $f$. Sendo $\left\{\eta_{i}\right\}_{i=1}^{m}$ os geradores de $\Theta_{V}$, temos:

Proposição C.0.30 $\left(\mathcal{K}_{V}\right.$-Espaço Tangente-[11]). Dada $f: \mathbb{C}^{s}, 0 \rightarrow \mathbb{C}^{t}$, temos que o espaço tangente à órbita de $f$ segundo a ação de $\mathcal{K}_{V}$ é dado por:

$$
T \mathcal{K}_{V} f=\mathcal{M}_{s}\left\{\frac{\partial f}{\partial x_{i}}\right\}+\mathcal{O}_{s}\left\{\eta_{i} \circ f\right\},
$$

e seu espaço tangente estendido é dado por:

$$
T \mathcal{K}_{V e} f=\mathcal{O}_{s}\left\{\frac{\partial f}{\partial x_{i}}\right\}+\mathcal{O}_{s}\left\{\eta_{i} \circ f\right\} .
$$




\section{Referências Bibliográficas}

[1] V. I. Arnold. Matrices depending on parameters. Uspehi Mat. Nauk, 26(2(158)):101-114, 1971.

[2] V. I. Arnold. Normal forms of functions near degenerate critical points, the Weyl groups $A_{k}, D_{k}, E_{k}$ and Lagrangian singularities. Funkcional. Anal. i Priložen., 6(4):3-25, 1972.

[3] V. I. Arnold. Critical points of functions on a manifold with boundary, the simple Lie groups $B_{k}, C_{k}, F_{4}$ and singularities of evolutes. Uspekhi Mat. Nauk, 33(5(203)):91-105, 237, 1978.

[4] D. Avritzer and H. Lange. Pencils of quadrics, binary forms and hyperelliptic curves. Comm. Algebra, 28(12):5541-5561, 2000. Special issue in honor of Robin Hartshorne.

[5] J. W. Bruce. On families of symmetric matrices. Mosc. Math. J., 3(2):335-360, 741, 2003. Dedicated to Vladimir I. Arnold on the occasion of his 65th birthday.

[6] J. W. Bruce and F. Tari. On binary differential equations. Nonlinearity, 8(2):255-271, 1995.

[7] J. W. Bruce and F. Tari. On families of square matrices. Proc. London Math. Soc. (3), 89(3):738-762, 2004.

[8] J. W. Bruce and F. Tari. Dupin indicatrices and families of curve congruences. Trans. Amer. Math. Soc., 357(1):267-285 (electronic), 2005.

[9] James Damon. The unfolding and determinacy theorems for subgroups of $\mathcal{A}$ and $\mathcal{K}$. Mem. Amer. Math. Soc., 50(306):x+88, 1984.

[10] James Damon. Deformations of sections of singularities and Gorenstein surface singularities. Amer. J. Math., 109(4):695-721, 1987.

[11] James Damon. $\mathcal{A}$-equivalence and the equivalence of sections of images and discriminants. In Singularity theory and its applications, Part I (Coventry, 1988/1989), volume 1462 of Lecture Notes in Math., pages 93-121. Springer, Berlin, 1991. 
[12] Terence Gaffney. Properties of finitely determined germs. PhD thesis, Brandeis University, 1976.

[13] C. G. Gibson. Singular points of smooth mappings, volume 25 of Research Notes in Mathematics. Pitman (Advanced Publishing Program), Boston, Mass., 1979.

[14] V. Goryunov and D. Mond. Tjurina and Milnor numbers of matrix singularities. $J$. London Math. Soc. (2), 72(1):205-224, 2005.

[15] Werner Greub. Multilinear algebra. Springer-Verlag, New York, second edition, 1978. Universitext.

[16] G. J. Haslinger. Families of skew-symmetric matrices. University of Liverpool Thesis, 2001.

[17] W. V. D. Hodge and D. Pedoe. Methods of algebraic geometry. Vol. II. Cambridge Mathematical Library. Cambridge University Press, Cambridge, 1994. Book III: General theory of algebraic varieties in projective space, Book IV: Quadrics and Grassmann varieties, Reprint of the 1952 original.

[18] Tadeusz Józefiak. Ideals generated by minors of a symmetric matrix. Comment. Math. Helv., 53(4):595-607, 1978.

[19] A. G. Kuz'min. Nonclassical equations of mixed type and their applications in gas dynamics, volume 109 of International Series of Numerical Mathematics. Birkhäuser Verlag, Basel, 1992. Translated and revised from the Russian by the author.

[20] Elon Lages Lima. Variedades diferenciáveis. Monografias de Matemática. Instituto de Matemática Pura e Aplicada, Rio de Janeiro, 1977.

[21] Flávio Ulhoa Coelho; Mary Lilian Lourenço. Um curso de álgebra linear. 2 edition, 2005.

[22] Jean Martinet. Singularities of smooth functions and maps, volume 58 of London Mathematical Society Lecture Note Series. Cambridge University Press, Cambridge, 1982. Translated from the French by Carl P. Simon.

[23] Marcos Sebastiani. Introdução à geometria analítica complexa. IMPA(Rio de Janeiro), 2004.

[24] F. Tari. Singularidades de aplicações diferenciáveis. Notas didáticas do ICMC, $n^{o} 34$, ICMC - USP - São Carlos, 1999.

[25] Farid Tari. Two parameter families of binary differential equations. Discrete Contin. Dyn. Syst., 22(3):759-789, 2008. 
[26] C. T. C. Wall. Finite determinacy of smooth map-germs. Bull. London Math. Soc., 13(6):481-539, 1981.

[27] C. T. C. Wall. Notes on the classification of singularities. Proc. London Math. Soc. (3), 48(3):461-513, 1984. 